\title{
Title: ANP32A regulates ATM expression and prevents oxidative stress in cartilage, brain and bone
}

Authors: Frederique M. F. Cornelis ${ }^{1 *}$, Silvia Monteagudo ${ }^{1 *}$, Laura-An K.A. Guns ${ }^{1}$, Wouter den Hollander ${ }^{2,3}$, Rob G.H.H. Nelissen ${ }^{4}$, Lies Storms ${ }^{1}$, Tine Peeters ${ }^{1}$, Ilse Jonkers ${ }^{1,5}$, Ingrid Meulenbelt ${ }^{2,3}$ and Rik J. Lories ${ }^{1,6 \dagger}$

\section{Affiliations:}

${ }^{1}$ Laboratory of Tissue Homeostasis and Disease, Skeletal Biology and Engineering Research Center, Department of Development and Regeneration, KU Leuven, Leuven, Belgium.

${ }^{2}$ Department of Medical Statistics and Bioinformatics, section Molecular Epidemiology, Leiden University Medical Center, Leiden, The Netherlands.

${ }^{3}$ Integrated research of Developmental determinants of Ageing and Longevity (IDEAL), Leiden, Netherlands.

${ }^{4}$ Department of Orthopaedics, Leiden University Medical Center, Leiden, The Netherlands.

${ }^{5}$ Human Movement Biomechanics, Department of Kinesiology, KU Leuven, Leuven, Belgium.

${ }^{6}$ Division of Rheumatology, University Hospitals Leuven, Belgium.

*These authors contributed equally to this work as co-first authors.

†To whom correspondence should be addressed: Rik.Lories@kuleuven.be

One Sentence Summary: This study identifies ANP32A as a key transcriptional regulator of the $A T M$ gene and therefore of the cell's response against oxidative stress thus providing protection against joint, neurological and bone disease. 
Abstract:

Osteoarthritis is the most common joint disorder with increasing global prevalence due to ageing of the population. Current therapy is limited to symptom relief, yet there is no cure. Its multi-factorial etiology includes oxidative stress and overproduction of reactive oxygen species, but the regulation of these processes in the joint is insufficiently understood. Here, we report that ANP32A protects the cartilage against oxidative stress, preventing osteoarthritis development and disease progression. ANP32A is downregulated in human and mouse osteoarthritic cartilage. Microarray profiling revealed that, mechanistically, ANP32A protects the joint by promoting the expression of ATM, a key regulator of the cellular oxidative defense. Indeed, antioxidant treatment reduces the severity of osteoarthritis in Anp32a-deficient mice. Remarkably, in these mice, we also observed cerebellar ataxia features responsive to antioxidant treatment, and osteopenia. Thus, the ANP32A/ATM axis discovered in cartilage is also present in brain and bone. In conclusion, our findings indicate that modulating the ANP32A network could provide novel opportunities to manage oxidative stress in cartilage, brain and bone with therapeutic implications for osteoarthritis, neurological disease and osteoporosis. 


\section{Introduction}

Osteoarthritis is the most common chronic joint disease worldwide. Patients with this musculoskeletal disorder suffer from pain and progressive loss of joint function and mobility. It therefore features among the leading causes of chronic disability, is strongly linked with ageing and the obesity pandemic and affects more than $25 \%$ of the adult population (1). Morphological, biochemical, molecular and biomechanical changes of the cells and extracellular matrix in the tissues of the joint lead to loss of articular cartilage, sclerosis of the subchondral bone, formation of bone spurs called osteophytes and, in some cases, inflammation of the synovial membrane that lines the joint cavity (2). The molecular mechanisms involved in the initiation and progression of osteoarthritis are insufficiently understood. Thus, there are no effective interventions to restore degraded cartilage, and to delay or halt disease progression (3). Indeed, current treatment strategies are limited to changes in lifestyle, appropriate exercise and use of painkillers and antiinflammatory drugs. Eventually, joint replacement surgery is often required, especially for the large joints $(4,5)$.

The etiology of osteoarthritis is multi-factorial and includes a strong genetic component adding to the deleterious effects of joint injury, obesity and ageing (6). Understanding the biological mechanisms underlying the genetic susceptibility could not only provide novel insights into disease pathogenesis, but also lead to the development of new therapies that slow or stop the progression of the disease. We described an association between polymorphisms in the acidic leucine rich nuclear phosphoprotein-32A $(A N P 32 A)$ gene and osteoarthritis $(7,8)$. ANP32A is a small multifunctional protein that plays a role in a variety of cellular processes such as caspase modulation, chromatin modifications, protein phosphatase inhibition and intracellular transport of molecules (9), thereby regulating cell differentiation, transcription, apoptosis and cell cycle 
progression (10-13). However, the potential role of ANP32A in osteoarthritis and cartilage biology remains unknown.

Here, we report that ANP32A protects against the development and progression of osteoarthritis by preventing oxidative stress in the articular cartilage. We also unravel the underlying molecular mechanism, which involves transcriptional control of the ataxia-telangiectasia mutated (ATM) gene, an important regulator of the cellular defense against oxidative stress (14). Additionally, the regulatory role of ANP32A and its underlying mechanism discovered in cartilage are also present in brain and bone. Thus, our results may not only have therapeutic implications in chronic joint disorders but also in bone and neurological diseases.

\section{Results}

\section{ANP32A levels are decreased in osteoarthritic and ageing cartilage}

To test whether the levels of $A N P 32 A$ are different between osteoarthritic and non-osteoarthritic joints, we performed gene expression analysis on human articular cartilage. ANP32A expression levels were reduced in hip cartilage from patients with osteoarthritis compared to trauma patients with macroscopically intact cartilage, both undergoing hip replacement surgery (Fig. 1A; for patient characteristics, see Table S1). These observations were confirmed at the protein level by Western blot (Fig. 1B). Immunohistochemical analysis similarly showed that the strong immunoreactive signal obtained in control non-osteoarthritic cartilage was decreased in cartilage from patients with osteoarthritis (Fig. 1C). Furthermore, in patients with knee and hip osteoarthritis, $A N P 32 A$ expression levels were reduced in damaged as compared to preserved areas of the articular cartilage (Fig. 1D). Similarly, immunoreactivity for ANP32A was decreased in the articular cartilage of wild-type mice with osteoarthritis triggered by surgical destabilization of the 
medial meniscus (DMM) compared to sham-operated animals (Fig. 1E), and in the articular cartilage of 12-month old mice compared to 8-week old mice (Fig. 1F). Taken together, these findings suggest that high ANP32A levels are positively associated with cartilage health.

\section{Loss of Anp32a increases severity of and susceptibility to osteoarthritis in mice}

To study the impact of ANP32A deficiency on articular cartilage, we challenged Anp32a $a^{-/-}$mice (mice with a global gene deletion of ANP32A) in different models of osteoarthritis. Anp32a $a^{-/-}$mice showed increased cartilage damage compared to wild-type controls in the gradually progressive DMM disease model driven by joint instability, in the rapidly progressive model triggered by intraarticular collagenase injection driven by severe instability and inflammation, and in the direct cartilage matrix damage model induced by intra-articular papain injection (Fig. 1G,H and fig. S1, A-D). ANP32A deficiency also predisposes to an accelerated development of spontaneously occurring osteoarthritic changes upon ageing, as $A n p 32 a^{-/-}$mice developed more severe signs of osteoarthritis by 12 months of age compared to wild-type mice (Fig. 1I,J). Collectively, these data indicate that loss of ANP32A increases the severity of and the susceptibility to osteoarthritis.

\section{ANP32A regulates Atm expression and oxidative stress in cartilage}

To explore the mechanisms underlying the impact of ANP32A on the onset and progression of osteoarthritis, we compared genome-wide transcriptome profiles from the articular cartilage of Anp32a $a^{-/}$mice and wild-type mice at 8-weeks of age (geonr: GSE108036). At this age, no significant differences in the expression of healthy chondrocyte markers collagen 2 or aggrecan were detected between the groups (fig. S2A). In Anp32a-/- mice, expression levels of 118 genes were different compared to control mice when applying a threshold of 2-fold change and a nominal $p$-value $\leq 0.01$ (Fig. 2A). The gene set included 106 downregulated genes and 12 upregulated 
genes (top ranked genes are shown in Table S2). Comparative pathway analyses of differentially regulated transcripts using the PANTHER database revealed an enrichment in genes associated with the oxidative stress response and the ubiquitin proteasome pathway (Fig. 2B), known to play a pivotal role in the recognition and degradation of oxidized proteins (15). Biological process categories found to be enriched in PANTHER analysis included DNA metabolic process, phosphate-containing compound metabolic process, catabolic process and cellular protein modification process (fig. S3). In addition, reactome pathways found to be enriched include mitochondrial translation, regulation of p53 and oxidative stress induced senescence (fig. S3). Thus, the transcriptome analysis suggests that oxidative stress and dysregulation of phosphorylation events play a role in the deleterious effects associated with ANP32A deficiency in cartilage.

Notably, in a prominent position within the top significantly downregulated genes, we identified the ataxia telangiectasia mutated serine threonine kinase $(\mathrm{Atm})$ gene (Fig. 2A, Table S2). Atm encodes a protein kinase that orchestrates signaling cascades to preserve the cellular redox balance and promote the antioxidant response (14). Downregulation of Atm in Anp32a $a^{-/-}$compared to wildtype mice was confirmed by qPCR (Fig. 2C). We also detected a considerable reduction of ATM protein levels in articular chondrocytes and in the articular cartilage of $A n p 32 a^{-/-}$mice compared to controls, analyzed by Western blot and immunohistochemistry, respectively (Fig. 2D,E). This decrease in ATM levels paralleled enhanced ROS production in the articular cartilage of Anp $32 a^{-}$ I- mice, assessed by immunohistochemistry and dihydroethidium (DHE) staining (Fig. 2F,G). We then measured the mRNA and protein expression levels of ATM in cartilage from nonosteoarthritic trauma patients and in cartilage from patients with osteoarthritis. We observed that ATM expression was downregulated in cartilage from patients with osteoarthritis compared to 
non-osteoarthritic cartilage (Fig. 2H, I) and in damaged areas compared to preserved areas (Fig. 2J), paralleling the changes in $A N P 32 A$ (Fig. 1A,B,D). Levels of $A N P 32 A$ and $A T M$ strongly correlated (Fig. 2K). Moreover, ANP32A knockdown in primary healthy human articular chondrocytes using siRNA (Fig. 2L) reduced $A T M$ expression levels (Fig. 2M). In ANP32A knockdown cells, $\mathrm{H}_{2} \mathrm{O}_{2}$ treatment increased ROS production more strongly than in control cells, an effect that was rescued by treatment with recombinant ATM (Fig. 2N). Overall, our results show a close link between ANP32A levels, oxidative stress and ATM expression and function in the articular cartilage.

The antioxidant system is complex and comprises multiple interacting and interdependent mechanisms (16). Thus, we investigated the eventual existence of compensatory mechanisms in the articular cartilage of the Anp32a-- mice using our transcriptome data. We thereby focused primarily on molecules involved in the regulation of glutathione, the most abundant endogenous antioxidant, on the peroxiredoxin and thioredoxin families, and catalase and the superoxide dismutases (Table S3). None of the individual genes analysed met our microarray thresholds of fold change and $P$-value. At the group level, overall changes in gene expression suggest an increase in enzymes responsible for glutathione synthesis and reduction (fig. S4). Thus, these data suggest that oxidative stress triggered by loss of ANP32A may result in some compensatory increase in the glutathione system that seems to be however insufficient to restore the cellular redox balance.

\section{ANP32A directly promotes $A T M$ expression in articular chondrocytes}

Given the strong link found between ANP32A and ATM, we investigated whether ANP32A directly regulates the $A T M$ gene at the transcriptional level. To address this question, we studied the binding of ANP32A to the $A T M$ gene promoter in primary healthy human articular chondrocytes. Chromatin immunoprecipitation-quantitative PCR (ChIP-qPCR) demonstrated that 
ANP32A localized at the $A T M$ gene promoter (Fig. 3A). Furthermore, to determine if ANP32A actively participates in the regulation of $A T M$ transcription, we evaluated the binding of the RNA polymerase II transcription machinery to the $A T M$ promoter after siRNA-mediated knockdown of ANP32A in human articular chondrocytes compared to controls. We observed that the recruitment of RNA polymerase II to the $A T M$ promoter was reduced in $A N P 32 A$ knockdown cells (Fig. 3B). Thus, these data indicate that ANP32A directly promotes the transcription of the $A T M$ gene in articular chondrocytes.

Oxidative stress induced by $\mathrm{H}_{2} \mathrm{O}_{2}$ treatment increased $A T M$ gene expression (Fig. 3C) and protein levels (Fig. 3D) in articular chondrocytes. In particular, the enhancement in ATM protein levels was observed in the cytoplasmic fraction (Fig. 3E), in agreement with the reported role of extranuclear ATM in oxidative stress defense co-localizing with peroxisomes, a major site of oxidative metabolism (17-19). Remarkably, oxidative stress induced by $\mathrm{H}_{2} \mathrm{O}_{2}$ treatment triggered a rapid translocation of ANP32A protein to the nucleus (Fig. 3E) and increased enrichment of ANP32A binding to the $A T M$ gene promoter (Fig. 3F). These results suggest that ANP32A may prevent osteoarthritis development and progression by increasing ATM levels in the articular cartilage as an endogenous protective mechanism against oxidative stress.

\section{Antioxidant treatment prevents osteoarthritis in Anp32a-deficient mice}

To explore the therapeutic implications of our findings, we evaluated whether pharmacological antioxidant treatment may have a protective effect on the development of osteoarthritis in Anp32adeficient mice. To this end, we administered the ROS inhibitor N-acetyl-cysteine (NAC) in Anp $32 a^{-/-}$mice and controls, via the drinking water. Pharmacological treatment with NAC effectively reduced the severity of osteoarthritis in Anp32a-deficient mice in the DMM model (Fig. 4A,B) and prevented ROS production in the articular cartilage of these mice (Fig. 4C). 
Atm-deficient mice have high ROS levels in growth plate chondrocytes, which leads to a proliferation defect and the stimulation of chondrocyte hypertrophy (20). We observed increased immunohistochemical staining of the hypertrophy marker type X collagen (COLX) in the growth plates of Anp32a-deficient mice compared to controls, and this effect was normalized by NAC treatment (fig. S5A,B). Osteoarthritis is associated with ectopic hypertrophic differentiation of chondrocytes in the articular cartilage. Altered matrix composition and factors secreted by these hypertrophic-like articular chondrocytes, such as MMP-13, likely contribute to cartilage degeneration (21). Therefore, we evaluated if Anp32a-deficient mice showed increased hypertrophic differentiation in the articular cartilage in the DMM model, as a consequence of the high ROS production. Effectively, we detected increased COLX staining in the articular cartilage of Anp32a-deficient mice compared to controls (Fig. 4D,E ). Antioxidant treatment also prevented chondrocyte hypertrophy in the articular cartilage of Anp32a-deficient mice (Fig. 4D,E). Thus, ANP32A seems to prevent ROS-induced ectopic chondrocyte hypertrophy in the articular cartilage. Together, these data further demonstrate that prevention of oxidative stress is the pivotal mechanism by which ANP32A protects cartilage against osteoarthritis.

\section{Anp32a deficiency leads to ataxia-like neurological defects that are attenuated by antioxidant treatment}

We then explored whether our insights into the role of ANP32A in cartilage and its discovered link with ATM may have clinical implications beyond this tissue, in particular in the context of ataxia telangiectasia (A-T). Patients with A-T lack functional ATM protein and exhibit a pleiotropic phenotype that includes cerebellar ataxia, immunodeficiency and premature ageing (22). Most of the clinical manifestations of A-T seem to result from an inability to limit the production of ROS (23). We evaluated the expression levels of Atm in Anp32a $a^{-/ 2}$ mouse brain, 
which was strongly downregulated compared to wild-type mice (Fig. 5A). At the protein level, ATM was strongly downregulated in the cerebellum of Anp32a $a^{-/-}$mice (Fig. 5B). Of note, we also observed increased ROS production in the cerebellum of Anp32a-/-mice compared to controls (Fig. $5 C)$.

To identify motor impairments in Anp32a $a^{-/-}$mice, we performed CatWalk gait analysis (Fig. 5DG), to reveal subtle coordination defects related to cerebellar dysfunction $(24,25)$. This approach suggested the presence of ataxia in Anp32 $a^{-/-}$mice as their stepping pattern was less consistent than in control mice. The average distance between each stride (stride length) was significantly shorter in Anp32a $2 a^{-/-}$mice compared with wild-type controls (Fig. 5E,F). Anp32a $a^{-/-}$mice also showed a significantly lower regularity index (Fig. 5E,G). Additional parameters of the CatWalk analysis that were significantly altered in Anp32a-- mice are shown in Table S4. Consistent with the observations in Atm-deficient mice (26,27), Anp32a-/- mice appeared to have a normal cerebellar architecture, and did not show histological signs of neuronal degeneration (fig. S6).

We then studied if antioxidant treatment also protected $A n p 32 a^{-/-}$mice from the development of ataxia-like features using oral administration of NAC (Fig. 5C-G), a drug earlier demonstrated to cross the blood-brain barrier $(28,29)$ and effective in reducing oxidative stress in the brain at the dosage used (30). Effectively, oral NAC supplementation in Anp32a $a^{-/}$mice from the age of 3 weeks until mice were 8-weeks old (Fig. 5D) effectively decreased the production of ROS in the cerebellum (Fig. 5C) and strongly ameliorated the gait disturbances associated with loss of ANP32A (Fig. 5E-G and Table S4). Interestingly, an exploratory experiment, albeit with limitations linked to the number of mice included, suggested that pharmacological intervention with NAC in Anp32a-deficient mice at an older age, from 8-months to 12-months old, also 
ameliorated the gait impairments (fig. S7). Thus, ataxia-like features in Anp32a/- mice can be linked to loss of ATM and excessive ROS production.

Although unlikely, it may be possible that the development of osteoarthritis in the mutant mice is not primarily caused by the absence of ANP32A in the cartilage. Impairments resulting from ataxia in the mutants may lead to different joint loading that could induce osteoarthritis features. However, silencing of ANP32A in non-osteoarthritic human articular chondrocytes resulted in decreased expression levels of healthy cartilage markers type II collagen and aggrecan (fig. S2B), supporting the existence of a cell-autonomous effect in the development of osteoarthritis. Further analysis of the gait experiments suggested that, while intensity of weight support seemed to decrease in the mutant mice, in particular in the front paws at an early age, stance duration consistently and significantly increased in both front and hind limbs. Consequently, the impulse, accounting for loading magnitude and duration, may be increased and therefore overall loading could at least be comparable and probably higher in Anp32a/- mice compared to wild-type mice. Of note, stance duration was significantly decreased by NAC treatment. Thus, an additional effect of ataxia on the development of osteoarthritis in the mice cannot be entirely excluded.

\section{Anp32a deficiency leads to osteopenia in mice that is prevented by antioxidant treatment}

$\mathrm{Atm}^{-/-}$mice exhibit an osteopenic bone phenotype, which is mainly due to impaired bone formation $(31,32)$. To further validate the link between ANP32A and ATM, we examined whether Anp32a deficiency also affected bone homeostasis. Effectively, screening of 12-week old Anp32a $a^{-/-}$mice, using dual energy X-ray absorptiometry (DEXA), demonstrated significantly reduced bone mineral density, bone mineral content and lean body mass compared to wild-type littermates (Fig. 6A). Further ex vivo analysis by peripheral quantitative computed tomography (pQCT) of femora from 12-week old Anp32a $a^{-/-}$mice confirmed decreased trabecular bone content, density and area 
as well as decreased cortical bone content and area (Fig. 6B). These observations were corroborated by histology of the tibiae dissected from 16-week old mice (Fig. 6C). These data support that the ANP32A-ATM axis also protects bone homeostasis by limiting ROS production. Of note, an exploratory analysis by in vivo $\mu \mathrm{CT}$ suggested that NAC intervention may also improve bone health in the Anp32a-deficient mice (Fig. 6D). Further end-stage analysis by histomorphometry however could not provide clear and consistent confirmation for this observation (Fig. 6C,E).

\section{Discussion}

In this study, we demonstrate that ANP32A maintains cartilage homeostasis and protects against the development of osteoarthritis based on the analysis of in vivo models of post-traumatic and age-related joint disease. ANP32A's protective role can be attributed to promoting the expression of $A T M$ in the articular cartilage, to preserve the cellular redox balance (fig. S8) - a molecular mechanism suggested by genome-wide microarray analysis on articular cartilage from Anp32a $a^{-/}$ mice and further elucidated using primary human articular chondrocytes. The ANP32A-ATM axis prevents the excessive accumulation of reactive oxygen species (ROS) that may directly damage DNA and proteins by oxidation and alter gene transcription programs. We further provide evidence that the regulatory role of ANP32A on Atm expression and on oxidative stress is not limited to cartilage but also exists in brain and bone. Effectively, Anp32a deficiency leads to ataxia-like cerebellar dysfunction and osteopenia. The identified disease-causing cascade of events seems to be responsive to therapeutic intervention as articular cartilage damage and ataxia are effectively prevented and ameliorated by oral antioxidant treatment. 
We discovered a novel and remarkable role for ANP32A as a positive transcriptional regulator of the Atm gene. This stimulatory function of ANP32A on gene transcription markedly contrasts with its earlier-defined role as transcriptional repressor. Indeed, ANP32A was first identified as a transcriptional repressor upon purification and characterization of the inhibitor of histone acetyltransferase (INHAT) complex, a multiprotein cellular complex that potently inhibits specific histone acetyltransferases (33). Further mechanistic insights revealed that ANP32A blocks histone acetylation by binding to histone tails and sterically inhibiting the histone-modifier enzymes ( 34 , 35). Other reports suggested that ANP32A represses transcription upon its recruitment to gene promoters by different transcription factors (36-38). Of note, in the trancriptome analysis of articular cartilage from Anp $32 a^{-/-}$mice compared to controls presented here, most of genes that significantly changed were downregulated, pointing out the unexpected role of ANP32A as positive regulator of transcription in cartilage cells. To our knowledge, only one study reported ANP32A to enhance gene transcription, in particular of interferon (IFN)-stimulated genes (39). ANP32A has been portrayed as a multifunctional protein that, in addition to the above-highlighted role in regulation of gene transcription, also inhibits phosphorylases, modulates caspase activity and affects intracellular transport (9). However, the specific effects on joint homeostasis reported here, do appear to be largely dependent on ANP32A's direct positive involvement in the regulation of $A T M$ expression, as demonstrated by the chromatin immunoprecipitation analysis and the different rescue experiments performed.

Our results reveal a novel endogenous system to control oxidative stress in cartilage. Indeed, we demonstrate that ANP32A stimulates ATM protein production in the articular cartilage as a protective mechanism to enhance the antioxidant capacities of the chondrocyte. In addition to its known role as a sensor of DNA double-strand breaks and thereby as regulator in the DNA damage 
response, the ATM kinase is involved in the regulation of the global cellular response against oxidative stress $(40,41)$. It is known that under oxidative stress conditions, ATM forms active homodimers (23) and manages stress via various pathways, including modulation of MAPK pathways, rerouting of carbon metabolism from glycolysis to the pentose phosphate pathway (PPP) thereby increasing the production of NADPH that is essential in the antioxidant defense and nucleotide synthesis, and repression of mTOR that leads to decreased mitochondrial activity and subsequent ROS production (40). In our study, loss of Anp32a resulted in strong downregulation of Atm and increased ROS production. Earlier studies have clearly demonstrated that excessive ROS contribute to the onset and progression of osteoarthritis (42). Furthermore, ROS production and oxidative stress are elevated in patients with osteoarthritis (43-45), and conversely, antioxidant enzymes are reduced in the joint of patients with osteoarthritis, confirming the role of oxidative stress in disease pathogenesis (44-48).

The articular cartilage is not vascularized, and consequently its oxygen supply is limited. Nevertheless, oxygen from the synovial fluid does diffuse into articular cartilage, and articular chondrocytes possess mitochondria and respire in vitro, producing ROS (49). In normal conditions, ROS are produced at low levels in articular chondrocytes and have a homeostatic function, regulating gene expression, ECM synthesis and breakdown, cytokine production and chondrocyte apoptosis (50). However, excessive ROS levels trigger oxidative damage of cellular proteins and also oxidize lipids, carbohydrates and DNA in human articular cartilage (51). Moreover, high levels of ROS contribute to cartilage degradation by inhibiting matrix synthesis, cell migration and growth factor bioactivity, by directly degrading matrix components, activating matrix metalloproteinases (MMPs) and inducing cell death (51). Given the dual role of ROS in cartilage, it is translationally relevant to gain insights into how ROS levels are regulated. 
Several factors may contribute to excessive ROS production in the onset and progression of osteoarthritis. Chondrocytes can produce abnormal levels of ROS in response to local oxygen variations $(52)$, mechanical stress $(53,54)$ and pro-inflammatory cytokines such as IL-1 $\beta(55)$. Ageing, a key risk factor for the development of osteoarthritis, is associated with elevated oxidative damage of DNA, proteins, and lipids, and accumulating evidence indicates that oxidative stress is a major physiological inducer of ageing (56). We observed reduced levels of ANP32A in aged mouse cartilage and in human cartilage from patients with osteoarthritis, and we showed that Anp32-deficient mice develop spontaneous osteoarthritis upon ageing. Thus, ANP32A can be considered as a key coordinator of oxidative stress and ageing in the joint.

Both chondrocyte death as well as the loss of the specific molecular identity of the articular chondrocyte contribute to osteoarthritis. Differentiation of articular cartilage cells towards cells with molecular characteristics of growth plate hypertrophic chondrocytes results in the synthesis of an extracellular matrix that will be calcified and impairs the optimal biomechanical characteristics of the bone-cartilage unit in the joint (57). We effectively demonstrated increased chondrocyte hypertrophy in the articular cartilage of Anp32a-deficient mice. Loss of Atm expression and increased oxidative stress are again demonstrated to be the underlying mechanism. Morita et al. reported that ROS stimulate chondrocyte hypertrophy in growth plate cartilage, which can be prevented in vitro and in vivo by antioxidant treatment (20). Increased ROS levels in growth plate cartilage of Atm-deficient mice and antioxidant rescue of subsequent increased hypertrophy were also reported (20).

Antioxidants such as NAC have been successfully used to prevent chondrocyte death e.g. in explants after excessive loading stress (54) or in a rat model of osteoarthritis (58). NAC has also been successful in inhibiting hypertrophic differentiation of articular chondrocytes by oxidized- 
low density lipoprotein (LDL) (59). These observations are in line with our NAC rescue experiments in the Anp32a $a^{-/-}$mice. Interestingly, repeated overloading of cartilage and severe injury to the cartilage have been associated with oxidative stress and mitochondrial dysfunction $(60,61)$. Of note, NAC treatment seems to be effective in providing protection for the cartilage in these clinically and translationally relevant settings.

Our results further demonstrate that the ANP32A-ATM axis is not only present in cartilage but also identify ANP32A as critical regulator of Atm expression and oxidative stress in cerebellum and bone. Lack of Anp32a resulted in clinical signs of ataxia, and these abnormalities were prevented by antioxidant treatment in young mice. We also documented a positive effect of antioxidant pharmacological intervention in older mice. Different mouse models of Atm deficiency have been developed with the neurological phenotype apparently dependent on genetic background and on the mutation strategies used $(26,27)$. A specific Atm-mutant strain showed motor-learning deficits and histological changes in the cerebellum that recapitulate some of the abnormalities seen in ataxia-telangiectasia patients at early disease stages (62). Barlow et al. demonstrated that loss of Atm in mice resulted in oxidative damage in different tissues, including the cerebellum (63), an observation that was confirmed by Kamsler et al. (64). Antioxidant treatments were shown to prevent some of these changes in respective genetic mouse models of Atm deficiency $(65,66)$.

The phenotype of the Anp32a knockout mice reported here may appear to be in contrast with the observations published in a prior study, in which phenotypic screening of this mouse strain did not show specific abnormalities in the nervous system (67). Indeed, no ataxia was detected in the Anp32a-deficient mouse strain (67), although behavioral analysis to assess cerebellar function was only done by Rotarod tests, a method that is likely less sensitive to detect abnormalities than the 
Catwalk System that we used (24). Moreover, the Anp32a-deficient mice used in our study were fully backcrossed to the C57Bl/6 background, which might not have been the case for mice reported earlier (67).

Oxidative stress is known to be a risk factor for the development and progression of osteoporosis (68). Lack of Anp32a also resulted in bone loss in line with a similar phenotype observed in Atmdeficient mice and attributed mostly to defective osteoblast differentiation and lack of bone anabolism $(31,32)$. Our results suggest that potentiation of the ANP32A-ATM axis in bone could enhance the antioxidant capacities of the cells. This could be translationally relevant in conditions such as in perimenopausal women, where hormonal changes are associated with increased oxidative stress $(69,70)$.

Some limitations apply to our study and its results. First, in the context of a translational study requiring large amounts of primary human cells from healthy controls, access to young undamaged human cartilage is extremely difficult. Our control samples were obtained from elderly individuals suffering an osteoporotic or pathological fracture for whom hip prosthesis surgery was required. Thus, despite macroscopically appearing healthy, the tissue and cells have aged and may be different from cartilage in young individuals. Nevertheless, gene expression and protein analyses clearly differentiated these samples from those of patients, which were from a similar age group but requiring prosthesis surgery for osteoarthritis of the hip. Second, animal models of osteoarthritis, in particular in rodents, mimic specific aspects of the disease dependent on the trigger and are therefore not identical to the human disorder. For further development of therapeutic approaches, large animal models in which the cartilage thickness and architecture is more closely related to that of humans, will likely be required. Third, in vivo experiments were performed with mice with a global deletion of the Anp32a gene. Thus, as noted above, the 
independence of the phenotypes in the different tissues cannot be assumed, in particular with regards to the impact of ataxia on osteoarthritis development. However, in the clinical literature there appears no evidence of a relationship between primary ataxia and the development of osteoarthritis. To minimize mouse handeling, NAC treatments were given by the drinking water. Drinking ad libitum may not allow a fully controlled individual application, but did permit to rescue the impact of ANP32A loss. NAC rescue was effectively demonstrated for the osteoarthritis and ataxia phenotype, but could not be convincingly shown for the osteoporosis in $A n p 32 a^{-/-}$mice. This may be related to high variability in the bone parameter measurements at the older age, the relative lack of statistical power due to the limited number of mice, or inadequate dosing or penetrance into the tissue of NAC to have rescue effects on the bone.

Although we demonstrate the important role of the ANP32A-ATM axis in protecting joint, brain and bone from oxidative stress, our experimental approaches are not likely to address the full complexity of the regulation of the redox status in cells and tissues, in particular the enzymatic activities in the system. At the gene expression level, our microarray data suggest the presence of some compensatory mechanisms, in particular in the regulation of gluthatione, the major cellular antioxidant system. Yet, this upregulation and putative post-translational mechanisms that impact the activity of the different redox enzymes did clearly not restore the redox balance altered by the loss of ANP32A.

In conclusion, our study identifies the ANP32A-ATM axis as a critical regulator of oxidative stress in different tissues and organs, thereby suggesting ANP32A as a therapeutic target for diseases associated with oxidative stress. Further research should focus on the factors that regulate ANP32A expression and activity in the articular cartilage and other tissues, in order to define 
additional therapeutic approaches to prevent cartilage damage and osteoarthritis, bone loss and osteoporosis, and cerebellar loss of function and ataxia. 


\section{Materials and Methods}

Study design. The objective of this study was to determine the role of ANP32A in the pathophysiology of osteoarthritis in humans and mice. Cartilage tissue and cells from patients with osteoarthritis and non-arthritic controls and genetically engineered mice were used in ex vivo studies, in different models of aging and joint disease, combined with in vitro assays. For all experiments, the largest possible sample size was used. In the osteoarthritis models an effect size of 2 with alpha error 0.05 and power 0.80 suggested at least 6 mice per group as sufficient for the analysis. No statistical method was used to predetermine the sample size for the ageing experiments on cartilage, brain and bone. No exclusion of animals was carried out. Mice were randomly assigned to the experimental groups where applicable. Pathology analysis was performed in a blinded fashion.

Patient materials. Human articular chondrocytes were isolated from the hips of patients undergoing total hip replacement surgery. For patient characteristics, see Table S1. The University Hospitals Leuven Ethics Committee and Biobank Committee approved the study (S56271) and specimens were taken with patients' written consent. Under Belgian Law and UZ Leuven's biobank policies, the hip joints are considered human biological residual material. Only age and sex of the patients are being shared between the surgeons and the investigators involved in this study. The material is fully anonymized without links to the medical file. Non-osteoarthritic articular chondrocytes used as controls were obtained from patients undergoing hip replacement for osteoporotic or malignancy-associated fractures. Upon arrival of the specimen, these nonosteoarthritis hips were macroscopically evaluated to exclude signs of osteoarthritis. Non- 
osteoarthritis hips were obtained from the division of traumatology, osteoarthritic samples were obtained from the division of orthopedics. For samples used in RNA sequencing, ethical approval was obtained from the medical ethics committee of the LUMC (P08.239) and informed consent was obtained from all participants $(71,72)$. Herein, participant details can be found.

Mice. Anp32a-- mice were a kind gift from Dr. P. Opal (Northwestern University Medical School, Chicago, USA) (67) and were backcrossed onto the C57Bl/6 background. In the experiments reported here, mice were at least in the $11^{\text {th }}$ generation of backcrossing. Wild-type Anp $32 a^{+/+}$ littermates or wild-type C57Bl/6, purchased from Janvier (Le Genest St Isle, France), were used as controls. All studies were performed with the approval from the Ethics Committee for Animal Research (KU Leuven, Belgium).

Mouse genotyping. Genotypes of animals were confirmed by polymerase chain reaction (PCR). Genotyping primers were designed to distinguish $A n p 32 a^{+/+}, A n p 32 a^{+/-}$and $A n p 32 a^{-/}$alleles: fw 1 ACAGCAAAGCCTACTGGATA and rev1 ATGTTGGATAAGCACACCTC pair: 401bp $\begin{array}{llll}\left(\text { Anp } 32 a^{+}\right. & \text {allele); fw1 ACAGCAAAGCCTACTGGATA and rev2 }\end{array}$ AGGAACCACAGGATGCTTCA pair: $601 \mathrm{bp}$ (Anp32a- allele). PCR reactions were performed as described (67).

Mouse osteoarthritis models. In the DMM model, mild instability of the knee was induced by transection of the medial menisco-tibial ligament of the right knee of male 8-week old mice (73). Knees were analyzed 12 weeks after surgery. In this model the contra-lateral knee was used as a control and sham surgery was performed in control groups. For the spontaneous development of 
osteoarthritis, Anp32a $a^{-/}$female mice and WT littermates were sacrificed at the age of 12 months. Arthritis induced by collagenase, is obtained by intra-articular injection of collagenase type VII ( 2 $\mu \mathrm{g} / \mu \mathrm{l}$; C0773; Sigma-Aldrich) in the right knee of male 8-week old mice. Knees were analyzed 21 days after the injection of collagenase. Arthritis induced by papain injection, is obtained by intraarticular injection of papain (1\% solution in $10 \mu \mathrm{l}$; P4762; Sigma-Aldrich) $+0,03 \mathrm{M}$ L-cysteine in the right knee of male 8-week old mice. Knees were analyzed 7 days after papain injection.

In vivo pharmacological NAC treatment. N-acetyl-cysteine (NAC) (Zambon S.A./N.V.) was added to the drinking water at a concentration of $1 \mathrm{mg} / \mathrm{ml}$ and mice were allowed to drink ad libitum. The solution was refreshed 3 times per week and protected from light. In the rescue experiment of the cartilage damage, male mice received NAC for 11 weeks, starting from the second week after the DMM surgery. In the rescue experiment of the ataxia phenotype at early age, male mice received NAC from weaning age (3 weeks) till the age of 16 weeks. In the rescue experiment of the ataxia phenotype at later age, male mice received NAC from 8 months of age till the age of 1 year. In the rescue experiment of the osteopenia phenotype analysed by histology and histomorphometry, male mice received NAC from weaning age (3 weeks) till the age of 16 weeks. In the rescue experiment of the osteopenia phenotype analysed by Micro-Computed Tomography $(\mu \mathrm{CT})$, female mice received NAC from 3 months of age till the age of 6 months.

Histology of the mouse joint. Mice were sacrificed, and tibia and knees were fixed in $2 \%$ formaldehyde overnight at $4{ }^{\circ} \mathrm{C}$; decalcified for 3 weeks in $0,5 \mathrm{M}$ EDTA $\mathrm{pH}$ 7,5; and embedded in paraffin or OCT. Haematoxylin-Eosin, Haematoxylin-SafraninO staining and immunohistochemistry was performed on $5 \mu \mathrm{m}$ thick sections. Dihydroethidium staining was 
performed on $5 \mu \mathrm{m}$ thick cryosections. Pictures were taken using a Visitron Systems microscope (Leica Microsystems GmbH) using Spot32 software. Severity of disease was determined by histological scores on Haematoxylin-SafraninO stained sections throughout the knee (5 sections at $100 \mu \mathrm{m}$ distance). Both cartilage damage and synovial hyperplasia were assessed based on OARSI guidelines $(73,74)$. For cartilage damage in the collagenase- and DMM-induced osteoarthritis models and in the spontaneous osteoarthritis model, only depth of lesion (0-6) was scored on frontal knee sections (73). Lesion grades represent the following features; 0 : surface and cartilage morphology intact, 1: small fibrillations without loss of cartilage, 2: vertical clefts below superficial layer and some loss of surface lamina, 3-6: vertical clefts/erosions to the calcified cartilage extending 3: less than $25 \%, 4: 25-50 \%, 5: 50-75 \%$ and 6 : more than $75 \%$. For cartilage damage in the papain model, both depth of lesions (score 0-6) and extent of lesions (score 0-4) on frontal knee sections were evaluated in a combined score (depth x extent) (74). Lesion grades represent the following features; 0: surface and cartilage morphology intact, 1: superficial zone fibrillation, 2: surface discontinuity, 3: vertical fissures, 4: cartilage erosions, 5: denudation of cartilage and exposure of subchondral bone and 6: remodeling including microfractures and osseus repair above the previous surface. Extent scores represent; 0: no lesions, 1: less than 10\%, 2: 10 to 25\%, 3: $25-50 \%$ and $4:$ over $50 \%$. Medial and lateral tibial and femoral cartilage was scored and the score represents the mean of the four quadrants. Scoring was done blinded to the genotype.

Histology of the mouse cerebellum. Mice were sacrificed, and brains were fixed in $4 \%$ formaldehyde overnight at $4^{\circ} \mathrm{C}$ and embedded in paraffin. Immunohistochemistry was performed on $5 \mu \mathrm{m}$ thick sections. Pictures were taken using a Visitron Systems microscope (Leica Microsystems GmbH) using Spot32 software. 
Immunohistochemistry. Immunohistochemistry was performed on paraffin embedded EDTA decalcified mouse knee sections, on paraffin embedded sections of human cartilage explants from osteoarthritic patients and control trauma patients, and on non-decalcified brain sections. First, sections were treated with $3 \% \mathrm{H}_{2} \mathrm{O}_{2}$ /water for 10 minutes to inactivate endogenous peroxidase activity. Then, the sections were blocked in normal goat or donkey serum for 30 minutes and incubated overnight at $4{ }^{\circ} \mathrm{C}$ with the primary antibodies against ATM (Ab82512, Abcam,

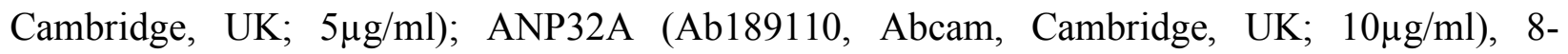
Hydroxydeoxyguanosine (8-OHdG) (Ab10802, Abcam, Cambridge, UK; 1:100), Calbindin (Ab11426, Abcam, Cambridge, UK; $1 \mu \mathrm{g} / \mathrm{ml}$ ), COLX (Ab58632, Abcam, Cambridge, UK; 1:250). After incubation with the primary antibody, 1:100 peroxidase goat anti-rabbit IgG or donkey antigoat IgG (Jackson Immunoresearch, Suffolk, UK) was applied for 30 minutes and peroxidase activity was determined using DAB (Dako, Carpinteria, CA, USA). Rabbit or goat IgG (Santa Cruz Biotechnologies, Santa Cruz, CA, USA) was used as negative controls. For the detection of ANP32A, heat induced epitope retrieval was performed using a Citrate-EDTA buffer (pH 6.0) for $10 \mathrm{~min}$ at $98^{\circ} \mathrm{C}$. For the detection of COLX, antigen retrieval was performed enzymatically with $10 \mathrm{mg} / \mathrm{ml}$ Hyaluronidase (Sigma Aldrich, H3884) in $\mathrm{MgCl} 2$ - free PBS, for $40 \mathrm{~min}$ at $37^{\circ} \mathrm{C}$. In this case, endogenous peroxidase activity was blocked after the incubation with the primary antibody. For the detection of Calbindin, antigen retrieval was performed via heat induced epitope retrieval using an EDTA buffer ( $\mathrm{pH} \mathrm{8.0)} \mathrm{for} 10 \mathrm{~min}$ at $98^{\circ} \mathrm{C}$. An amplification step using the Vectastain ABC kit (Vector Laboratories, Burlingame, CA, USA) was used for the detection of ANP32A and COLX. Quantification of the DAB immunohistochemical staining was performed with color deconvolution plugin (Jacqui Ross, Auckland University) in ImageJ Software (NIH Image, 
National Institutes of Health, Bethesda, MD, USA). Quantification was performed using two technical replicates for 3 different mice per condition, obtained in 3 different staining experiments.

Dihydroethidium staining. Dihydroethidium (DHE) (Invitrogen, D-23107) was used to detect ROS production in chondrocytes from male $A n p 32 a^{-/}$and wild-type C57Bl/6 mice. First the cryosections were incubated for 7 minutes at $37^{\circ} \mathrm{C}$ in the dark, and then rinsed twice in phosphate buffered saline (PBS). These cryosections were incubated with $30 \mu \mathrm{M}$ DHE for 30 minutes at room temperature. After rinsing the sections in PBS, a coverslip was mounted in Mowiol (Calbiochem) supplemented with 4', 6-Diamidino-2-phenylindole dihydrochloride (Dapi; Sigma Aldrich) diluted 1:1000.

Catwalk gait analysis. Male Anp $32 a^{-/}$and $\mathrm{C} 57 \mathrm{Bl} / 6$ controls were evaluated for gait abnormalities using the Catwalk Gait Analysis system (Noldus, Catwalk 7.1) available at the KU Leuven Small animal imaging core facility (MOSAIC), at the age of 8 and 16 weeks, and at 10 and 12 months. In this system, the mouse traverses a glass plate voluntarily (towards a goal box), and its footprints are captured by a camera system and analysed using the Noldus Catwalk 7.1 software (24).

Microarray hybridization and data acquisition. The articular cartilage and subchondral bone from the tibial plateau of the mouse knee joint was carefully dissected in one piece and was homogenized in TRIZOL (Invitrogen), using the Fastprep-24 tissue-homogenizer (MP Biomedicals, Solon, OH, USA). RNA was isolated using a manual phenol/chloroform extraction protocol. RNA concentration and purity were assessed with a NanoDrop Spectrophotometer (Nano Drop Technologies, Centreville, DE, USA) and integrity (RIN) was determined using RNA 
nanochips and the Agilent 2100 Bio-analyzer (Agilent Technologies, Diegem, Belgium). Only samples with acceptable quality and integrity (RIN above 7) were selected for the experiment. Microarrays were performes with Affymetrix Mouse 4302.0 chips, using 4 C57B1/6 wild-type and 4 Anp $32 a^{-/}$(8 week-old) male mouse RNA samples. A comparable analysis was previously performed for $\mathrm{Frzb}^{-/}$mice (75). Microarray data have been deposited in the Gene Expression Omnibus (GEO) public database (http://www.ncbi.nlm.nih.gov/geo/) and are accessible through GEP number (data submitted). The differentially expressed genes were explored with the limma package. PANTHER database version 11.1 (http://pantherdb.org) (76) was used for pathway analysis and graphical representation of functional categories.

Quantitative PCR. Total RNA was isolated using a manual phenol/chloroform extraction protocol or with the NucleoSpin RNA extraction kit (Macherey-Nagel, Filterservices) according to the manufacturer's instructions. cDNA was synthetized using $1 \mu \mathrm{g}$ total RNA with the RevertAid H Minus First Strand cDNA Synthesis Kit (Fermentas) according to the manufacturer's recommendations. The relative expression level of transcripts was determined by real-time RTPCR using cDNA as a template with Maxima SYBR Green qPCR Master Mix (Fermentas), following manufacturer's instructions and gene-specific primers on a Corbett Rotor-gene qPCR machine (Qiagen). Relative quantification was obtained with the $\Delta \Delta \mathrm{ct}$ method using Hprt as internal control, unless otherwise specified. Primers used in quantitative PCR are listed in Table S5.

Dual energy X-ray absorptiometry (DEXA). Total body (subcapital) bone density, lean and fat body mass from 12-weeks old female Anp32a $a^{-/}$mice and wild-type littermates were determined 
in vivo by Dual energy X-ray absorptiometry using a Piximus densitometer (Luna, Madison, WI, USA).

Peripheral quantitative computed tomography (pQCT). Trabecular and cortical bone mineral content, density and area of the femur from 12-weeks old female Anp32a $a^{-/}$mice and wild-type littermates were assessed ex vivo by peripheral quantitative computed tomography using an XCT Research M+ system (Norland Medical Systems, Trumbull, CT, USA). Slices of 0,2 mm in thickness were scanned using a voxel size of $0,07 \mathrm{~mm}$. Three scans were taken 2,4 - 2,6 mm from the distal end of the femur to determine trabecular bone parameters. An additional scan was taken $4 \mathrm{~mm}$ from the distal end of the femur to determine cortical bone parameters.

$\boldsymbol{\mu C T}$. Mice were sedated with $2 \%$ isoflurane $/ 21 \mathrm{O}_{2}$ flow inhalation anesthesia by a nose cone and scanned with a desktop in vivo small animal Micro-Computed Tomography ( $\mu$ CT) (Skyscan 1076, software version 3.2, Kontich, Belgium). The right hind-limb from female Anp32a $a^{-/}$mice was scanned consecutively at 3 months and 6 months of age. Images were acquired 'in-air' with the following parameters: $50 \mathrm{kV}$ X-ray source voltage, $100 \mu \mathrm{A}$ current, a composite X-ray filter of $1 \mathrm{~mm}$ Aluminium, $3300 \mathrm{msec}$ camera exposure time per projection, acquiring projections with $1^{\circ}$ increments over a total angle of $199^{\circ}$, producing images with a pixel size of $9 \mu \mathrm{m}$. Total scanning time was approximately $12 \mathrm{~min}$. Tomograms were constructed using NRecon software (version 1.6.2.0, Skyscan). Reconstruction parameters were smoothing ' 0 ', beam-hardening correction '30\%', ring artifact correction ' 8 ', maximum density range '0,118'; and misalignment compensation was automatically adapted for each individual scan. Trabecular and cortical volumes of interest of the tibia were hand-drawn and histomorphometric parameters were analysed using 
CTAn software (version 1.10.0.0, Skyscan). The proximal growth plate of the tibia was used as a reference point. For the trabecular bone, the offset and height were respectively 10 and 150 slices; for the cortical bone, the offset and height were respectively 500 and 100 slices. For the analysis of the trabecular bone, adaptive mean $\mathrm{C}$ tresholding was used with parameters: radius ' 8 ', constant ' 0 ', low ' 45 ' and high ' 255 '; and white speckles less than 30 pixels were removed. For the analysis of the cortex, initially, global tresholding was used with parameters: low '70' and high '255'; black speckles less than 1000 pixels were removed in 2D space; the ROI shrink wrap was stretched in 2D space over holes with 20 pixels diameter; finally, global tresholding was used with parameters: low '0' and high ' 255 '.

Histomorphometry of the bone. Bone histomorphometry was performed on HaematoxylinSafraninO-stained sections using an Olympus IX83 microscope and digital image analysis using Osteomeasure software. To measure trabecular bone parameters of male Anp32a $a^{-/}$compared to C57Bl/6 WT mice at 16 month-old, the lowest point of the growth plate was used as a reference, then an offset of $250 \mu \mathrm{m}$ was taken into account. Next, a box of $1 \mathrm{~mm}^{2}$ aligning the medial cortical bone was created. All trabecular bone was drawn manually and calculated by the software. To measure cortical bone thickness, the lowest point of the growth plate was used as a reference. An offset of $750 \mu \mathrm{m}$ was taken into account. Next, a box with a width of $500 \mu \mathrm{m}$ and a height of $750 \mu \mathrm{m}$ overlapping the medial cortical bone was created. The cortical bone was aligned manually and the thickness was calculated by the software.

RNA sequencing of osteoarthritis human articular cartilage. Macroscopically preserved as well as macroscopically lesioned cartilage was sampled from patients who underwent a total joint 
replacement due to primary osteoarthritis of either the knee $(\mathrm{N}=15)$ or the hip $(\mathrm{N}=6)$ as part of the RAAK study (71). At the moment of collection (within 2 hours following surgery) tissue was washed extensively with PBS to decrease the risk of contamination by blood. Care was taken to avoid contamination with bone or synovium. Collected cartilage was snap frozen in liquid nitrogen and stored at $-80^{\circ} \mathrm{C}$ prior to RNA extraction. Cartilage samples were pulverized using a Retsch MM200 under cryogenic conditions. On average, $150 \mathrm{mg}$ of pulverized cartilage was dissolved in $1 \mathrm{ml}$ of Trizol reagent, and mixed vigorously. After addition of $200 \mu \mathrm{l}$ of chloroform the sample was mixed and centrifuged for 15 minutes $(16,000 \mathrm{~g})$. The clear aqueous layer was transferred to a new vial and 1 volume of $70 \%$ ethanol/DEPC-treated water was added to precipitate RNA. RNA was collected using Qiagen mini columns according to the manufacturer's protocol and quality was assessed using a Bioanalyzer lab-on-a-chip. RNA integrity numbers above 8 were considered suitable for RNA sequencing (72).

RNA-sequencing data. Post RNA isolation, paired-end 100 bp RNA library sequencing (Illumina TruSeq RNA Library Prep Kit, Illumina HiSeq 2000) resulted in an average of 10 million clusters. Reads were aligned using GSNAP against the hg19 reference genome. Using the edgeR package, fragments per gene were used to assess the dispersion by quantile-adjusted conditional maximum likelihood (qCML). Subsequently, differential gene expression analysis was performed pairwise between preserved and lesioned samples for which we had RNA of both $(\mathrm{N}=21)$ followed by FDR correction.

Cell Culture and transfection. Healthy articular chondrocytes were obtained from patients undergoing hip replacement for osteoporotic or malignancy-associated fractures. In specimens 
from osteoarthritic patients, obtained during prosthesis surgery, cartilage tissue was first classified macroscopically as either intact or damaged as described previously (77) taking into account color, surface integrity and tactile impression tested with a scalpel. Cartilage was dissected from the joint explant surfaces and then rinsed with PBS. The tissue was cut into small pieces, using a sterile surgical blade. Cartilage explants were incubated with $2 \mathrm{mg} / \mathrm{ml}$ pronase solution (Roche) for 90 minutes at $37^{\circ} \mathrm{C}$ and digested overnight at $37^{\circ} \mathrm{C}$ in $1.5 \mathrm{mg} / \mathrm{ml}$ collagenase $\mathrm{B}$ solution (Roche) under continuous agitation. The preparation was filtered through a $70 \mu \mathrm{M}$ strainer and cells were plated in culture flasks and cultured in a humidified atmosphere at $37^{\circ} \mathrm{C}, 5 \% \mathrm{CO}_{2}$. Culture medium consisted of DMEM/F12 (Gibco), 10\% fetal bovine serum (FBS) (Gibco), 1\% (vol/vol) antibiotic/antimycotic (Gibco) and 1\% L-glutamine (Gibco).

For the in vitro studies, cells were treated with $100 \mu \mathrm{M} \mathrm{H}_{2} \mathrm{O}_{2}$ (Chem Lab) or $0.5-1 \mu \mathrm{g} / \mathrm{ml}$ mouse recombinant ATM protein (14-933; Millipore). For small interfering RNA (siRNA) transfection, lipofectamin RNAiMAX (Invitrogen) was used as transfection reagent, together with ANP32a Stealth siRNA (Invitrogen) or negative control siRNA (Invitrogen), following the protocols provided by the manufacturer.

ChIP Analysis. Chromatin immunoprecipitation assays were carried out using the Agarose ChIP kit from Thermo Scientific, according to the manufacturer's guidelines. Briefly, cell samples were crosslinked by $1 \%$ Formaldehyde for $10 \mathrm{~min}$, and the reaction was stopped by the addition of glycine to a $125 \mathrm{mM}$ final concentration. The fixed cells were lysed in SDS buffer, and the chromatin was fragmented by microccocal nuclease digestion. The sheared chromatin was incubated with antibodies against ANP32A (Santa Cruz, sc-100767) and RNA polymerase II 
(Thermo Scientific, MA1-46093), and recovered by binding to protein A/G agarose. Eluted DNA fragments were used directly for qPCR. Primers used in quantitative PCR are listed in Table S3.

Subcellular fractionation and Western blotting. Cytoplasmic and nuclear extracts were prepared according to the instructions of the NE-PER ${ }^{\circledR}$ nuclear and cytoplasmic extraction kit (Pierce). The protein concentration of the cytoplasmic and nuclear extracts was determined by Pierce $^{\mathrm{TM}}$ BCA Protein Assay Kit (Thermo Scientific). Immunoblotting analyses were performed as described in previous studies ${ }^{3}$. Antibodies against Actin (Sigma, A2066; dilution 1:4000), total H3 (Abcam, ab1791; dilution 1:10000), ANP32A (Abcam, ab189110; dilution 1:1000) and ATM (Cell signaling, 2873S; dilution 1:1000) were used following manufacturer's instructions. The blotting signals were detected using the SuperSignalWest Femto Maximum Sensitivity Substrate system (Thermo Scientific).

DCFDA cellular ROS detection assay. The levels of intracellular ROS were detected by using a DCFDA Cellular ROS Detection Assay Kit (ab113851; Abcam, Cambridge, MA, USA) according to the manufacturer's instructions. In brief, after completion of the treatments, cell culture medium was removed and cells were washed with $4^{\circ} \mathrm{C}$ PBS, and they were then stained with DCFDA solution (final concentration: $30 \mu \mathrm{M}$ ) for 45 minutes at $37^{\circ} \mathrm{C}$ in the dark. Subsequently, fluorescence intensity was determined with a microplate reader (BioTek Synergy) (at excitation and emission wavelengths of $485 \mathrm{~nm}$ and $535 \mathrm{~nm}$, respectively).

\section{Statistical analysis}


Data are presented as mean and s.e.m. or as individual data points, representing the mean of technical replicates as indicated in the figure legends. Statistical analyses were performed where appropriate with R Studio (Version 1.0.15) and GraphPad Prism software. A detailed overview of the statistical analyses and assumptions is provided in Supplementary file 1. Data distribution was evaluated based on parameter characteristics, QQ plots and Shapiro-Wilk normality tests. Variances were compared using the Levene test. T-tests or ANOVA-tests were applied taking into account equal or different variances (applying Welch corrections). When different groups were compared by ANOVA tests, pair-wise $t$-tests were subsequently performed applying a Bonferroni correction for multiple comparisons to control for Type I errors in rejecting the null hypothesis. File S1 reports estimates of differences of means between groups (95\% confidence intervals). 


\section{Supplementary Materials}

\section{Supplementary figures}

Fig. S1. Loss of ANP32A increases the severity of osteoarthritis in the collagenase- and papaininduced mouse models.

Fig. S2. Expression of molecular markers associated with the healthy chondrocyte in the presence or absence of ANP32A.

Fig. S3. Transcriptome network analysis of articular cartilage of Anp32a-deficient mice.

Fig. S4. Compensatory regulation of anti-oxidant systems in the articular cartilage of Anp32adeficient mice.

Fig. S5. Immunohistochemistry of type X collagen levels in the growth plates of Anp32a compared to WT mice.

Fig. S6. Calbindin immunostaining of cerebellar Purkinje Cells of 16-week old WT and Anp32adeficient mice.

Fig. S7. Late stage antioxidant intervention in Anp32a-deficient mice ameliorates ataxia-related defects.

Fig. S8. Model for the role of ANP32A on oxidative stress.

\section{Supplementary tables}

Table S1. Patient characteristics. 
Table S2. Top ranked genes of transcriptome network of articular cartilage of Anp32a-deficient mice.

Table S3. Gene expression of main antioxidant in transcriptome network of articular cartilage of Anp32a-deficient mice.

Table S4. Gait parameters of early stage antioxidant intervention in Anp32a-deficient mice.

Table S5. Primers used in qPCR analysis. 


\section{References and Notes:}

1. D. J. Hunter, D. Schofield, E. Callander, The individual and socioeconomic impact of osteoarthritis. Nature Reviews Rheumatology 10, 437-441 (2014).

2. R. F. Loeser, S. R. Goldring, C. R. Scanzello, M. B. Goldring, Osteoarthritis: a disease of the joint as an organ. Arthritis and rheumatism 64, 1697-1707 (2012).

3. D. Chen, J. Shen, W. Zhao, T. Wang, L. Han, J. L. Hamilton, H. J. Im, Osteoarthritis: toward a comprehensive understanding of pathological mechanism. Bone research 5, 16044 (2017).

4. J. W. Bijlsma, F. Berenbaum, F. P. Lafeber, Osteoarthritis: an update with relevance for clinical practice. Lancet (London, England) 377, 2115-2126 (2011).

5. P. Cram, X. Lu, S. L. Kates, J. A. Singh, Y. Li, B. R. Wolf, Total knee arthroplasty volume, utilization, and outcomes among Medicare beneficiaries, 1991-2010. Jama 308, 1227-1236 (2012).

6. L. N. Reynard, J. Loughlin, Insights from human genetic studies into the pathways involved in osteoarthritis. Nature Reviews Rheumatology 9, 573-583 (2013).

7. A. M. Valdes, J. Loughlin, K. M. Timms, J. J. van Meurs, L. Southam, S. G. Wilson, S. Doherty, R. J. Lories, F. P. Luyten, A. Gutin, V. Abkevich, D. Ge, A. Hofman, A. G. Uitterlinden, D. J. Hart, F. Zhang, G. Zhai, R. J. Egli, M. Doherty, J. Lanchbury, T. D. Spector, Genome-wide association scan identifies a prostaglandin-endoperoxide synthase 2 variant involved in risk of knee osteoarthritis. American journal of human genetics 82, 1231-1240 (2008).

8. A. M. Valdes, R. J. Lories, J. B. van Meurs, H. Kerkhof, S. Doherty, A. Hofman, D. J. Hart, F. Zhang, F. P. Luyten, A. G. Uitterlinden, M. Doherty, T. D. Spector, Variation at the ANP32A gene is associated with risk of hip osteoarthritis in women. Arthritis and rheumatism 60, 2046-2054 (2009).

9. P. T. Reilly, Y. Yu, A. Hamiche, L. Wang, Cracking the ANP32 whips: Important functions, unequal requirement, and hints at disease implications. BioEssays 36, 1062-1071 (2014).

10. O. Adegbola, G. R. Pasternack, Phosphorylated retinoblastoma protein complexes with pp32 and inhibits pp32-mediated apoptosis. The Journal of biological chemistry 280, 15497-15502 (2005).

11. A. Theodosiou, A. Ashworth, MAP kinase phosphatases. Genome biology 3, Reviews3009.3001-3010 (2002).

12. T. A. Millward, S. Zolnierowicz, B. A. Hemmings, Regulation of protein kinase cascades by protein phosphatase 2A. Trends in biochemical sciences 24, 186-191 (1999).

13. D. Chakravarti, R. Hong, SET-ting the stage for life and death. Cell 112, 589-591 (2003).

14. K. R. Choy, D. J. Watters, Neurodegeneration in ataxia-telangiectasia: Multiple roles of ATM kinase in cellular homeostasis. Developmental Dynamics 247, 33-46 (2018).

15. F. Shang, A. Taylor, Ubiquitin-proteasome pathway and cellular responses to oxidative stress. Free Radical Biology and Medicine 51, 5-16 (2011).

16. C. Espinosa-Diez, V. Miguel, D. Mennerich, T. Kietzmann, P. Sánchez-Pérez, S. Cadenas, S. Lamas, Antioxidant responses and cellular adjustments to oxidative stress. Redox Biology 6, 183-197 (2015).

17. D. Watters, P. Kedar, K. Spring, J. Bjorkman, P. Chen, M. Gatei, G. Birrell, B. Garrone, P. Srinivasa, D. I. Crane, M. F. Lavin, Localization of a Portion of Extranuclear ATM to Peroxisomes. Journal of Biological Chemistry 274, 34277-34282 (1999).

18. D.-Q. Yang, M.-J. Halaby, Y. Li, J. C. Hibma, P. Burn, Cytoplasmic ATM protein kinase: an emerging therapeutic target for diabetes, cancer and neuronal degeneration. Drug Discovery Today 16, 332-338 (2011).

19. A. Alexander, C. L. Walker, Differential localization of ATM is correlated with activation of distinct downstream signaling pathways. Cell Cycle 9, 3709-3710 (2010).

20. K. Morita, T. Miyamoto, N. Fujita, Y. Kubota, K. Ito, K. Takubo, K. Miyamoto, K. Ninomiya, T. Suzuki, R. Iwasaki, M. Yagi, H. Takaishi, Y. Toyama, T. Suda, Reactive oxygen species induce chondrocyte hypertrophy in endochondral ossification. The Journal of Experimental Medicine 204, 1613-1623 (2007).

21. M. M.-G. Sun, F. Beier, Chondrocyte hypertrophy in skeletal development, growth, and disease. Birth Defects Research Part C: Embryo Today: Reviews 102, 74-82 (2014).

22. Y. Shiloh, ATM and related protein kinases: safeguarding genome integrity. Nature Reviews Cancer 3, 155-168 (2003).

23. Z. Guo, S. Kozlov, M. F. Lavin, M. D. Person, T. T. Paull, ATM Activation by Oxidative Stress. Science 330, $517-521$ (2010). 
24. C. Vandeputte, J.-M. Taymans, C. Casteels, F. Coun, Y. Ni, K. Van Laere, V. Baekelandt, Automated quantitative gait analysis in animal models of movement disorders. BMC Neuroscience 11, 92 (2010).

25. S. De Munter, S. Verheijden, E. Vanderstuyft, A. R. Malheiro, P. Brites, D. Gall, S. N. Schiffmann, M. Baes, Early-onset Purkinje cell dysfunction underlies cerebellar ataxia in peroxisomal multifunctional protein-2 deficiency. Neurobiology of Disease 94, 157-168 (2016).

26. C. Barlow, S. Hirotsune, R. Paylor, M. Liyanage, M. Eckhaus, F. Collins, Y. Shiloh, J. N. Crawley, T. Ried, D. Tagle, A. Wynshaw-Boris, Atm-Deficient Mice: A Paradigm of Ataxia Telangiectasia. Cell 86, 159-171 (1996).

27. Y. Xu, T. Ashley, E. E. Brainerd, R. T. Bronson, M. S. Meyn, D. Baltimore, Targeted disruption of ATM leads to growth retardation, chromosomal fragmentation during meiosis, immune defects, and thymic lymphoma. Genes \& Development 10, 2411-2422 (1996).

28. R. C. Reyes, G. F. Cittolin-Santos, J.-E. Kim, S. J. Won, A. M. Brennan-Minnella, M. Katz, G. A. Glass, R. A. Swanson, Neuronal Glutathione Content and Antioxidant Capacity can be Normalized In Situ by Nacetyl Cysteine Concentrations Attained in Human Cerebrospinal Fluid. Neurotherapeutics 13, 217-225 (2016).

29. A. L. Sheffner, E. M. Medler, K. R. Bailey, D. G. Gallo, A. J. Mueller, H. P. Sarett, Metabolic studies with acetylcysteine. Biochemical Pharmacology 15, 1523-1535 (1966).

30. D.-M. Otte, B. Sommersberg, A. Kudin, C. Guerrero, Ö. Albayram, M. D. Filiou, P. Frisch, Ö. Yilmaz, E. Drews, C. W. Turck, A. Bilkei-Gorzó, W. S. Kunz, H. Beck, A. Zimmer, N-acetyl Cysteine Treatment Rescues Cognitive Deficits Induced by Mitochondrial Dysfunction in G72/G30 Transgenic Mice. Neuropsychopharmacology 36, 2233-2243 (2011).

31. N. Rasheed, X. Wang, Q.-T. Niu, J. Yeh, B. Li, Atm-deficient mice: an osteoporosis model with defective osteoblast differentiation and increased osteoclastogenesis. Human Molecular Genetics 15, 1938-1948 (2006).

32. A. Hishiya, M. Ito, H. Aburatani, N. Motoyama, K. Ikeda, K. Watanabe, Ataxia telangiectasia mutated (Atm) knockout mice as a model of osteopenia due to impaired bone formation. Bone 37, 497-503 (2005).

33. S.-b. Seo, P. McNamara, S. Heo, A. Turner, W. S. Lane, D. Chakravarti, Regulation of Histone Acetylation and Transcription by INHAT, a Human Cellular Complex Containing the Set Oncoprotein. Cell 104, 119130 (2001).

34. R. Schneider, A. J. Bannister, C. Weise, T. Kouzarides, Direct Binding of INHAT to H3 Tails Disrupted by Modifications. Journal of Biological Chemistry 279, 23859-23862 (2004).

35. S.-b. Seo, T. Macfarlan, P. McNamara, R. Hong, Y. Mukai, S. Heo, D. Chakravarti, Regulation of Histone Acetylation and Transcription by Nuclear Protein pp32, a Subunit of the INHAT Complex. Journal of Biological Chemistry 277, 14005-14010 (2002).

36. M. Cvetanovic, R. J. Rooney, J. J. Garcia, N. Toporovskaya, H. Y. Zoghbi, P. Opal, The role of LANP and ataxin 1 in E4F-mediated transcriptional repression. EMBO reports 8, 671-677 (2007).

37. C. S. Hunter, R. E. Malik, F. A. Witzmann, S. J. Rhodes, LHX3 Interacts with Inhibitor of Histone Acetyltransferase Complex Subunits LANP and TAF-1 $\beta$ to Modulate Pituitary Gene Regulation. PLoS ONE 8, e68898 (2013).

38. M. A. Loven, R. E. Davis, C. D. Curtis, N. Muster, J. R. Yates, A. M. Nardulli, A Novel Estrogen Receptor $\alpha$-Associated Protein Alters Receptor-Deoxyribonucleic Acid Interactions and Represses ReceptorMediated Transcription. Molecular Endocrinology 18, 2649-2659 (2004).

39. S. Kadota, K. Nagata, pp32, an INHAT component, is a transcription machinery recruiter for maximal induction of IFN-stimulated genes. Journal of Cell Science 124, 892-899 (2011).

40. A. Guleria, S. Chandna, ATM kinase: Much more than a DNA damage responsive protein. DNA Repair 39, $1-20$ (2016).

41. A. Barzilai, G. Rotman, Y. Shiloh, ATM deficiency and oxidative stress: a new dimension of defective response to DNA damage. DNA Repair 1, 3-25 (2002).

42. P. Lepetsos, A. G. Papavassiliou, ROS/oxidative stress signaling in osteoarthritis. Biochimica et Biophysica Acta (BBA) - Molecular Basis of Disease 1862, 576-591 (2016).

43. M. A. Altay, C. Ertürk, A. Bilge, M. Yapt1, A. Levent, N. Aksoy, Evaluation of prolidase activity and oxidative status in patients with knee osteoarthritis: relationships with radiographic severity and clinical parameters. Rheumatology International 35, 1725-1731 (2015).

44. C. Ertürk, M. A. Altay, Ş. Selek, A. Koçyiğit, Paraoxonase-1 activity and oxidative status in patients with knee osteoarthritis and their relationship with radiological and clinical parameters. Scandinavian Journal of Clinical and Laboratory Investigation 72, 433-439 (2012). 
45. O. Altindag, O. Erel, N. Aksoy, S. Selek, H. Celik, M. Karaoglanoglu, Increased oxidative stress and its relation with collagen metabolism in knee osteoarthritis. Rheumatology International 27, 339-344 (2007).

46. A. Ostalowska, E. Birkner, M. Wiecha, S. Kasperczyk, A. Kasperczyk, D. Kapolka, A. Zon-Giebel, Lipid peroxidation and antioxidant enzymes in synovial fluid of patients with primary and secondary osteoarthritis of the knee joint. Osteoarthritis and Cartilage 14, 139-145 (2006).

47. E. A. Regan, R. P. Bowler, J. D. Crapo, Joint fluid antioxidants are decreased in osteoarthritic joints compared to joints with macroscopically intact cartilage and subacute injury. Osteoarthritis and Cartilage 16, 515-521 (2008).

48. M. Fernandez-Moreno, A. Soto-Hermida, S. Pertega, N. Oreiro, C. Fernandez-Lopez, I. Rego-Perez, F. J. Blanco, Mitochondrial DNA (mtDNA) haplogroups and serum levels of anti-oxidant enzymes in patients with osteoarthritis. BMC Musculoskeletal Disorders 12, 264 (2011).

49. R. B. LEE, J. P. G. URBAN, Evidence for a negative Pasteur effect in articular cartilage. Biochemical Journal 321, 95-102 (1997).

50. Y. E. Henrotin, P. Bruckner, J. P. L. Pujol, The role of reactive oxygen species in homeostasis and degradation of cartilage. Osteoarthritis and Cartilage 11, 747-755 (2003).

51. Y. Henrotin, B. Kurz, T. Aigner, Oxygen and reactive oxygen species in cartilage degradation: friends or foes? Osteoarthritis and Cartilage 13, 643-654 (2005).

52. D. R. Blake, J. Unsworth, J. M. Outhwaite, C. J. Morris, P. Merry, B. L. Kidd, R. Ballard, L. Gray, J. Lunec, Hypoxic-reperfusion injury in the inflamed human joint. The Lancet 333, 289-293 (1989).

53. M. Koike, H. Nojiri, Y. Ozawa, K. Watanabe, Y. Muramatsu, H. Kaneko, D. Morikawa, K. Kobayashi, Y. Saita, T. Sasho, T. Shirasawa, K. Yokote, K. Kaneko, T. Shimizu, Mechanical overloading causes mitochondrial superoxide and SOD2 imbalance in chondrocytes resulting in cartilage degeneration. Scientific Reports 5, 11722 (2015).

54. B. R. Beecher, J. A. Martin, D. R. Pedersen, A. D. Heiner, J. A. Buckwalter, Antioxidants Block Cyclic Loading Induced Chondrocyte Death. The Iowa Orthopaedic Journal 27, 1-8 (2007).

55. M. Mathy-Hartert, G. P. Deby-Dupont, J. Y. L. Reginster, N. Ayache, J. P. Pujol, Y. E. Henrotin, Regulation by reactive oxygen species of interleukin-1 $\beta$, nitric oxide and prostaglandin E2 production by human chondrocytes. Osteoarthritis and Cartilage 10, 547-555 (2002).

56. D. Liu, Y. Xu, p53, Oxidative Stress, and Aging. Antioxidants \& Redox Signaling 15, 1669-1678 (2011).

57. R. J. Lories, F. P. Luyten, The bone-cartilage unit in osteoarthritis. Nature Reviews Rheumatology 7, 43 (2010).

58. S. Nakagawa, Y. Arai, O. Mazda, T. Kishida, K. A. Takahashi, K. Sakao, M. Saito, K. Honjo, J. Imanishi, T. Kubo, N-acetylcysteine prevents nitric oxide-induced chondrocyte apoptosis and cartilage degeneration in an experimental model of osteoarthritis. Journal of Orthopaedic Research 28, 156-163 (2010).

59. H. Kishimoto, M. Akagi, S. Zushi, T. Teramura, Y. Onodera, T. Sawamura, C. Hamanishi, Induction of hypertrophic chondrocyte-like phenotypes by oxidized LDL in cultured bovine articular chondrocytes through increase in oxidative stress. Osteoarthritis and Cartilage 18, 1284-1290 (2010).

60. J. Riegger, H. Joos, H. G. Palm, B. Friemert, H. Reichel, A. Ignatius, R. E. Brenner, Antioxidative therapy in an ex vivo human cartilage trauma-model: attenuation of trauma-induced cell loss and ECM-destructive enzymes by N-acetyl cysteine. Osteoarthritis and Cartilage 24, 2171-2180 (2016).

61. M. C. Coleman, J. E. Goetz, M. J. Brouillette, D. Seol, M. C. Willey, E. B. Petersen, H. D. Anderson, N. R. Hendrickson, J. Compton, B. Khorsand, A. S. Morris, A. K. Salem, D. C. Fredericks, T. O. McKinley, J. A. Martin, Targeting mitochondrial responses to intra-articular fracture to prevent posttraumatic osteoarthritis. Science Translational Medicine 10, (2018).

62. P. R. Borghesani, F. W. Alt, A. Bottaro, L. Davidson, S. Aksoy, G. A. Rathbun, T. M. Roberts, W. Swat, R. A. Segal, Y. Gu, Abnormal development of Purkinje cells and lymphocytes in Atm mutant mice. Proceedings of the National Academy of Sciences of the United States of America 97, 3336-3341 (2000).

63. C. Barlow, P. A. Dennery, M. K. Shigenaga, M. A. Smith, J. D. Morrow, L. J. Roberts, A. Wynshaw-Boris, R. L. Levine, Loss of the ataxia-telangiectasia gene product causes oxidative damage in target organs. Proceedings of the National Academy of Sciences of the United States of America 96, 9915-9919 (1999).

64. A. Kamsler, D. Daily, A. Hochman, N. Stern, Y. Shiloh, G. Rotman, A. Barzilai, Increased Oxidative Stress in Ataxia Telangiectasia Evidenced by Alterations in Redox State of Brains from Atm-deficient Mice. Cancer Research 61, 1849-1854 (2001).

65. S. E. Browne, L. J. Roberts, P. A. Dennery, S. R. Doctrow, M. F. Beal, C. Barlow, R. L. Levine, Treatment with a catalytic antioxidant corrects the neurobehavioral defect in ataxia-telangiectasia mice. Free Radical Biology and Medicine 36, 938-942 (2004). 
66. N. Gueven, J. Luff, C. Peng, K. Hosokawa, S. E. Bottle, M. F. Lavin, Dramatic extension of tumor latency and correction of neurobehavioral phenotype in Atm-mutant mice with a nitroxide antioxidant. Free Radical Biology and Medicine 41, 992-1000 (2006).

67. P. Opal, J. J. Garcia, A. E. McCall, B. Xu, E. J. Weeber, J. D. Sweatt, H. T. Orr, H. Y. Zoghbi, Generation and characterization of LANP/pp32 null mice. Molecular and cellular biology 24, 3140-3149 (2004).

68. G. Hendrickx, E. Boudin, W. Van Hul, A look behind the scenes: the risk and pathogenesis of primary osteoporosis. Nature Reviews Rheumatology 11, 462 (2015).

69. M. J. Ramírez-Expósito, E. Sánchez-López, C. Cueto-Ureña, B. Dueñas, P. Carrera-González, J. NavarroCecilia, M. D. Mayas, J. M. Arias de Saavedra, R. Sánchez-Agesta, J. M. Martínez-Martos, Circulating oxidative stress parameters in pre- and post-menopausal healthy women and in women suffering from breast cancer treated or not with neoadjuvant chemotherapy. Experimental Gerontology 58, 34-42 (2014).

70. A. A. F. Pereira, K. G. Tirapeli, A. H. Chaves-Neto, M. da Silva Brasilino, C. Q. da Rocha, A. Belló-Klein, S. F. Llesuy, R. C. M. Dornelles, A. C. d. M. S. Nakamune, Ilex paraguariensis supplementation may be an effective nutritional approach to modulate oxidative stress during perimenopause. Experimental Gerontology 90, 14-18 (2017).

71. Y. F. M. Ramos, W. den Hollander, J. V. M. G. Bovée, N. Bomer, R. van der Breggen, N. Lakenberg, J. C. Keurentjes, J. J. Goeman, P. E. Slagboom, R. G. H. H. Nelissen, S. D. Bos, I. Meulenbelt, Genes Involved in the Osteoarthritis Process Identified through Genome Wide Expression Analysis in Articular Cartilage; the RAAK Study. PLoS ONE 9, e103056 (2014).

72. W. den Hollander, C. G. Boer, D. J. Hart, M. S. Yau, Y. F. M. Ramos, S. Metrustry, L. Broer, J. Deelen, L. A. Cupples, F. Rivadeneira, M. Kloppenburg, M. Peters, T. D. Spector, A. Hofman, P. E. Slagboom, R. G. H. H. Nelissen, A. G. Uitterlinden, D. T. Felson, A. M. Valdes, I. Meulenbelt, J. J. B. van Meurs, Genomewide association and functional studies identify a role for matrix Gla protein in osteoarthritis of the hand. Annals of the Rheumatic Diseases 76, 2046-2053 (2017).

73. S. S. Glasson, M. G. Chambers, W. B. Van Den Berg, C. B. Little, The OARSI histopathology initiative recommendations for histological assessments of osteoarthritis in the mouse. Osteoarthritis and Cartilage 18, S17-S23 (2010).

74. K. P. H. Pritzker, S. Gay, S. A. Jimenez, K. Ostergaard, J. P. Pelletier, P. A. Revell, D. Salter, W. B. van den Berg, Osteoarthritis cartilage histopathology: grading and staging. Osteoarthritis and Cartilage 14, 1329 (2006).

75. L. Lodewyckx, F. Cailotto, S. Thysen, F. P. Luyten, R. J. Lories, Tight regulation of wingless-type signaling in the articular cartilage - subchondral bone biomechanical unit: transcriptomics in Frzb-knockout mice. Arthritis Research \& Therapy 14, R16-R16 (2012).

76. H. Mi, X. Huang, A. Muruganujan, H. Tang, C. Mills, D. Kang, P. D. Thomas, PANTHER version 11: expanded annotation data from Gene Ontology and Reactome pathways, and data analysis tool enhancements. Nucleic Acids Research 45, D183-D189 (2017).

77. M. Geyer, S. Grässel, R. H. Straub, G. Schett, R. Dinser, J. Grifka, S. Gay, E. Neumann, U. Müller-Ladner, Differential transcriptome analysis of intraarticular lesional vs intact cartilage reveals new candidate genes in osteoarthritis pathophysiology. Osteoarthritis and Cartilage 17, 328-335 (2009). 


\section{Acknowledgments:}

We are grateful to A. Hens for taking care of the animal facility management. We also thank N. Dirckx for assistance with digital image analysis. We are indepted to the traumatology and orthopedic surgeons willing to contribute samples (A. Sermon, J.P. Simon and S. Nys) as well as the nursing staff of the surgical theater (in particular M. Penninckx). We thank all study participants of the RAAK study.

\section{Funding:}

This work was supported by grants from the Flanders Research Foundation (FWO-Vlaanderen), by the IUAP network Development and Repair (Belspo. Grant Number: IUAP-VII/07), by the FP7 project Translational Research in Europe Applied Technologies for Osteoarthritis (Treat-OA, European Commision framework 7 programme grant 200800), the Dutch Arthritis Association (DAA_10_1-402), the Dutch Scientific Research council NWO/ZonMW VICI scheme (nr. 91816631/528), the Leiden University Medical Center, and by a Marie-Curie Intra-European postdoctoral fellowships to S.M.

\section{Author contributions:}

R.J.L., F.M.F.C., S.M., I.M. and R.G.H.H.N. planned the study and designed experiments. F.M.F.C. S.M., L.-A.G., W. H., L. S and T. P. performed the experiments. S.M., F.M.F.C., R.J.L., I.M. and I.J. wrote the manuscript.

\section{Competing interests:}


The authors declare no competing financial interests.

Data and materials availability:

The data supporting the findings of this study are available within the article and its Supplementary Information files and from the corresponding author on reasonable request. 
Figures:

Figure 1

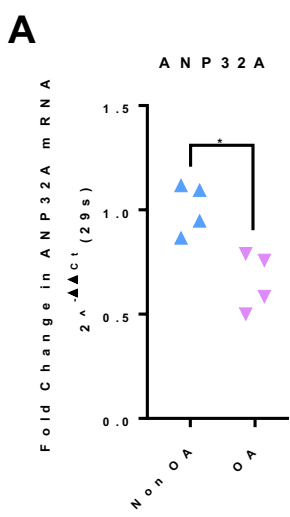

B
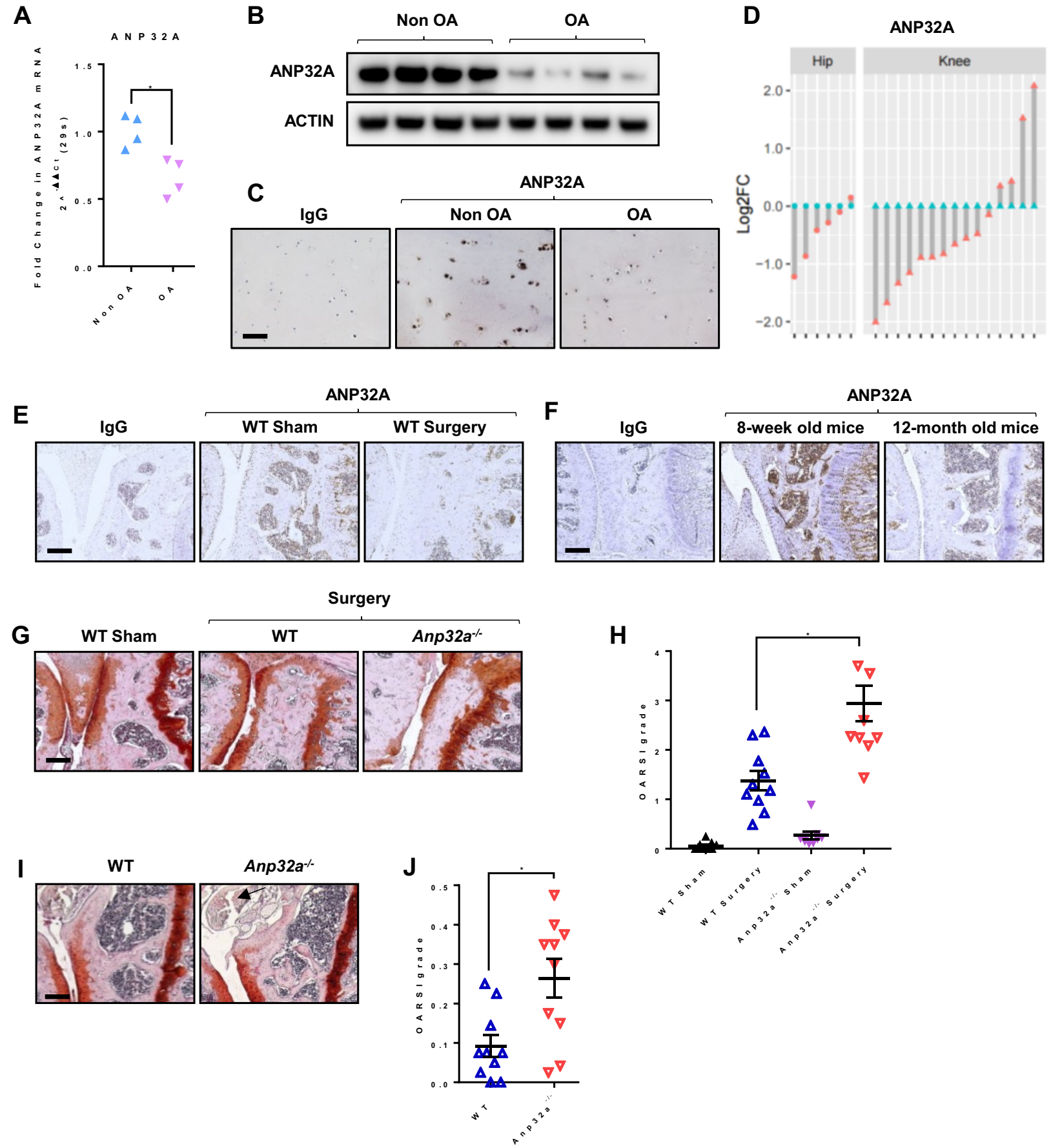

Fig. 1. Loss of ANP32A increases the severity of and the susceptibility to osteoarthritis. (A,B) Expression of $A N P 32 A$ measured by quantitative PCR (A) and by Western blot (B) in articular chondrocytes from patients with hip osteoarthritis (OA) $(n=4)$ as compared to chondrocytes from 
hip fracture patients without OA (non-OA) $(\mathrm{n}=4) .{ }^{*} P<0.01, t$-test, data are from one experiment with three technical replicates per patient sample. (C) Immunohistochemistry showing strong immunoreactivity for ANP32A in control non-OA cartilage and lower levels in cartilage from patients with OA. Images are representative of images from 4 different patients. Scale bar, 400 $\mu \mathrm{m}$. (D) Expression of $A N P 32 A$ determined by RNA sequencing in paired preserved and damaged cartilage isolated from hips (o) and knees $(\Delta)$ from patients with osteoarthritis. The values for the preserved cartilage were set at 0 for each cartilage pair and data presented as log2-fold change (LOG2FC). $(P=0<0.001 /$ False discovery rate $(\mathrm{FDR})=0,001 /$ mean relative fold change (FC) $=0.44)$. (E,F) Immunohistochemistry showing reduced ANP32A levels in wild-type (WT) mice 12 weeks after induction of osteoarthritis by destabilization of the medial meniscus (DMM) (WT Surgery) compared to sham-operated mice (WT Sham) (E); and in 8-week old mice as compared to 12-month old mice $(F)$. Images are representative of images from $5(E)$ and $3(F)$ different mice. Scale bar, $200 \mu \mathrm{m}$. (G) Hematoxylin-SafraninO staining of WT Sham mice, WT and Anp32a $a^{-/}$ mice 12 weeks after DMM. Scale bar, $200 \mu \mathrm{m}$. (H) Quantification of articular cartilage damage in the knees of Anp32a-/- mice and WT mice knee joints after DMM by OARSI severity grade score $\left({ }^{*} P<0.001\right.$, one-way ANOVA and Bonferroni corrected post test; $\mathrm{n}=8$ and 10). (I,J) Hematoxylin-SafraninO staining and quantification of articular cartilage damage of 12-month old Anp $32 a^{-/-}$mice and WT mice knee joints by OARSI severity grade score $\left({ }^{*} P<0.01, t\right.$-test; $\mathrm{n}=$ 10). Scale bar, $200 \mu \mathrm{m}$. Images are representative of images from 8 to 10 different mice. Error bars indicate mean \pm s.e.m. 
Figure 2

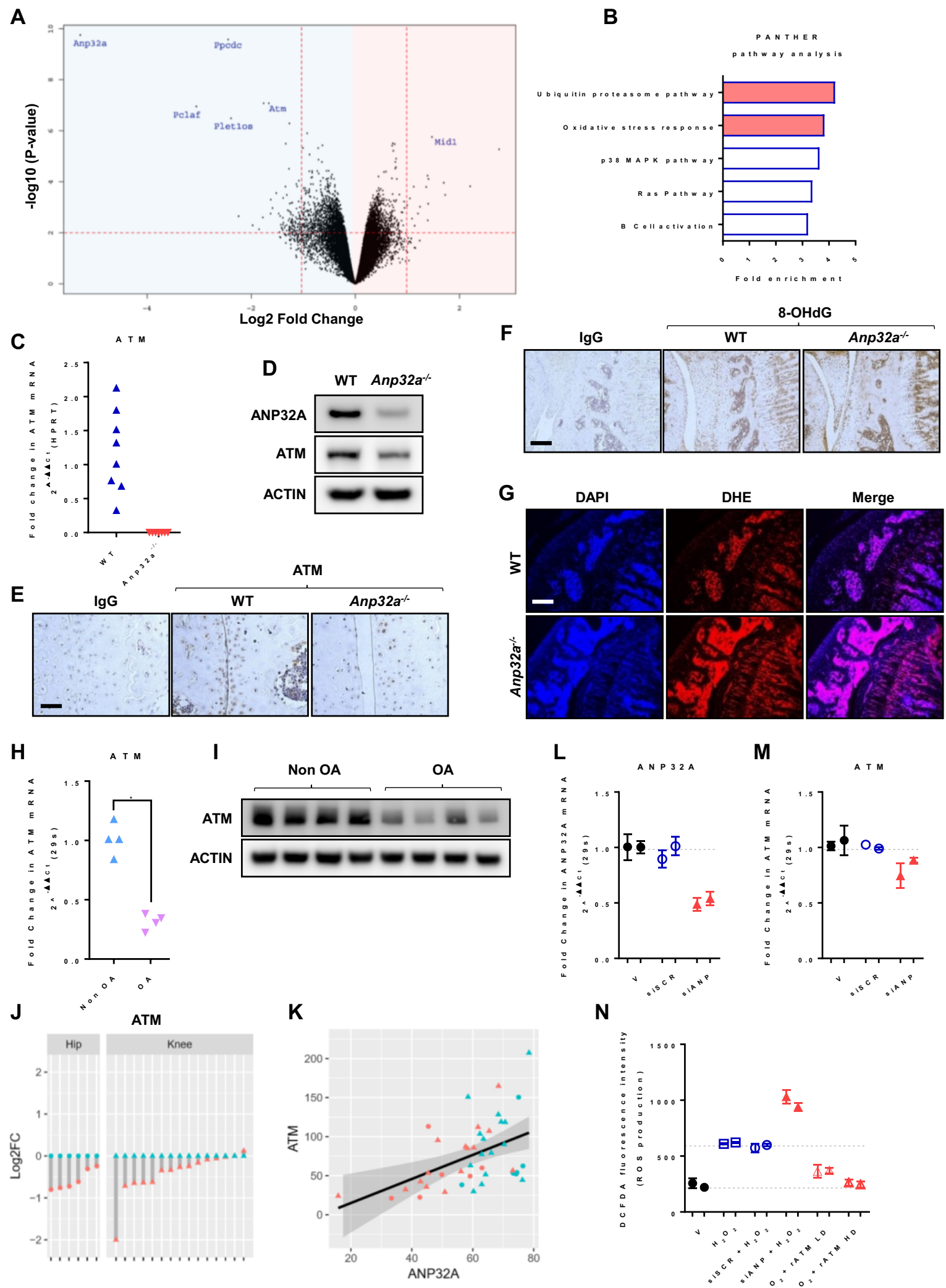


Fig. 2. ANP32A deficiency reduces ATM expression and triggers oxidative stress in cartilage. (A) Volcano plot of the microarray data obtained from tibial articular cartilage of Anp32a-deficient male mice and WT mice ( $n=4$ per group). The volcano plot shows the differentially expressed genes by $\log 2$ ratio (Anp32a-/ $/$ WT mice) as x-axis vs. - $\log 10$ ( $P$-values) as y-axis. (B) PANTHER pathway analysis of microarray data. (C,D) Quantitative PCR data (C) and Western blot analysis (D) confirming the downregulation of $A T M$ expression in articular chondrocytes from male Anp32a-deficient mice. (E) Immunohistochemistry showing reduced ATM levels in the articular cartilage of knees from 8-week old male Anp32a-deficient mice compared to WT mice. (F,G) ROS levels assessed by immunohistochemical detection of 8-hydroxydeoxyguanosine (8-OHdG) (F) and Dihydroethidium (DHE) staining (G), in 8-week old male knees from WT and Anp32adeficient mice. (H,I) Expression of ATM measured by quantitative PCR $(\mathrm{H})$ and by Western blot analysis (I) in articular chondrocytes from hips of osteoarthritic patients (OA) $(n=4)$ as compared to chondrocytes from non-OA patients $(\mathrm{n}=4) . * P<0.001$, $t$-test, data are from one experiment with three technical replicates per patient sample. (J) Expression of ATM determined by RNA sequencing in paired preserved and damaged cartilage isolated from hips (o) and knees $(\Delta)$ from patients with osteoarthritis. The values for the preserved cartilage was set at 0 for each cartilage pair. $P$-value $<0,01 /$ False discovery rate $(\mathrm{FDR})=0,008 /$ mean relative fold change $(\mathrm{FC})=0,48$. (K) Correlation between $A N P 32 A$ and $A T M$ expression levels. Pearson's correlation $=0.536 / P<$ 0.001. (L,M) $A N P 32 A$ and $A T M$ expression measured by quantitative PCR in human articular chondrocytes transfected with siRNA against $A N P 32 A$ (siANP) or scrambled siRNA (siSCR). Data are from two biologically different experiments each with three technical replicates. (N) ROS levels assessed by 2',7'-dichlorofluorescin diacetate (DCFDA) in human articular chondrocytes treated with $\mathrm{H}_{2} \mathrm{O}_{2}$ and recombinant ATM protein (rATM) (0.5 (low dose - LD) or 1 (high dose HD) $\mu \mathrm{g} / \mathrm{ml}$ ), and transfected with siANP or siSCR. Data are from two biologically different experiments each with three technical replicates. (E-G) The images are representative of images from 3 different mice. Error bars indicate mean \pm s.e.m. Scale bar, $200 \mu \mathrm{m}(\mathrm{F}, \mathrm{G})$ and $50 \mu \mathrm{m}(\mathrm{E})$. 
Figure 3

A

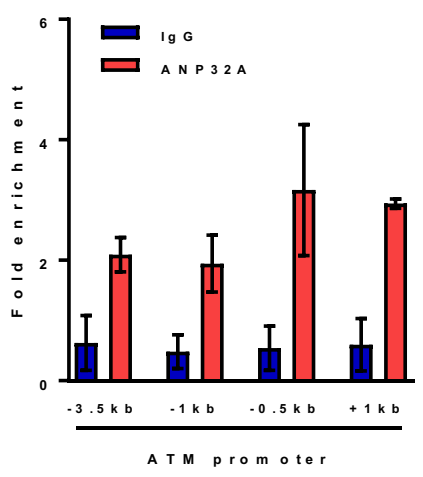

C

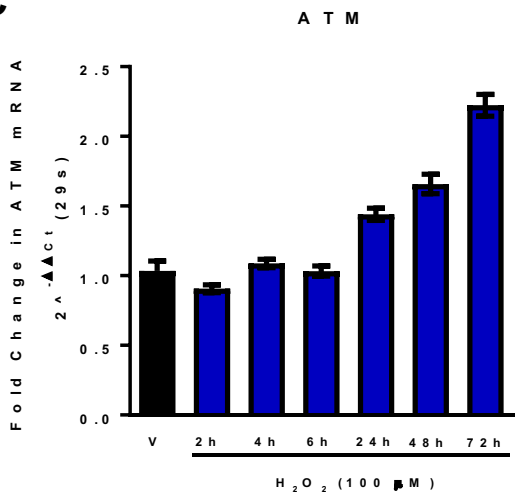

E
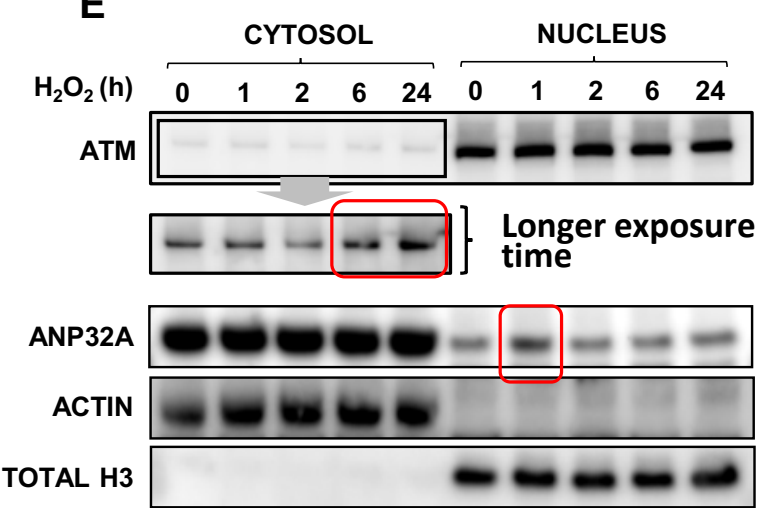

B

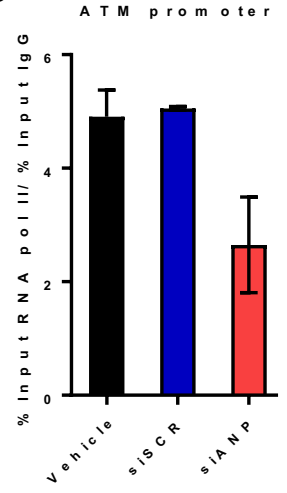

D

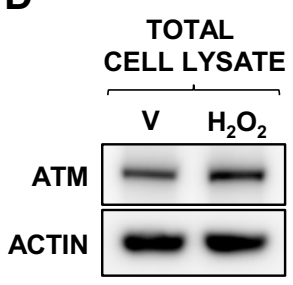

$\mathbf{F}$

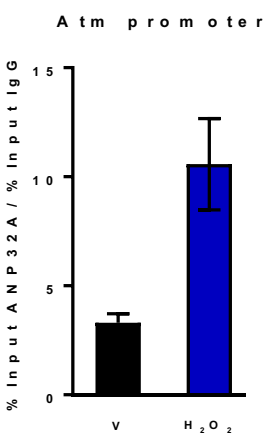

Fig. 3. ANP32A directly induces ATM expression to prevent oxidative stress in cartilage. (A) Chromatin-immunoprecipitation quantitative PCR (ChIP-qPCR) analysis of ANP32A binding to different regions of the ATM gene promoter in non-osteoarthritic human articular chondrocytes. (B) ChIP-qPCR analysis of RNA polymerase II binding to the ATM promoter in human articular chondrocytes transfected with siANP or scrambled siRNA. (C,D) Expression of ATM measured by quantitative PCR (C) and by Western blot analysis (D) in articular chondrocytes treated over 
time with $\mathrm{H}_{2} \mathrm{O}_{2}$ (C) or for $72 \mathrm{~h}$ (D). (E) Immunoblot analysis showing that $\mathrm{H}_{2} \mathrm{O}_{2}$ treatment increases cytosolic ATM protein levels and triggers rapid translocation of ANP32A to the nucleus in human articular chondrocytes. Cells were lysed and fractionated at the indicated times after adding $\mathrm{H}_{2} \mathrm{O}_{2}$. The image is representative of images from 3 independent experiments. (F) ChIP-qPCR analysis of ANP32A binding to the $A T M$ promoter showing an increased enrichment upon $\mathrm{H}_{2} \mathrm{O}_{2}$ treatment, in human articular chondrocytes. (A,B,F) Data from 2 biologically independent experiments. (C) Data are from two experiments with three technical replicates. Error bars indicate mean \pm s.e.m. 
Figure 4

A

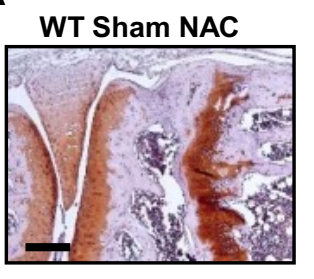

Surgery

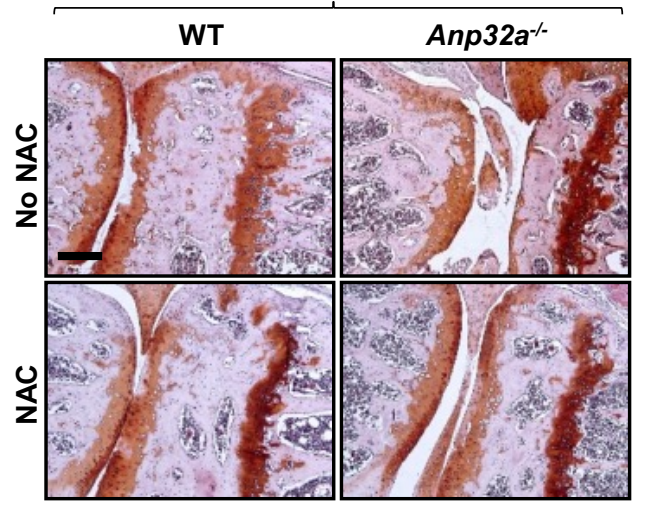

8-OHdG

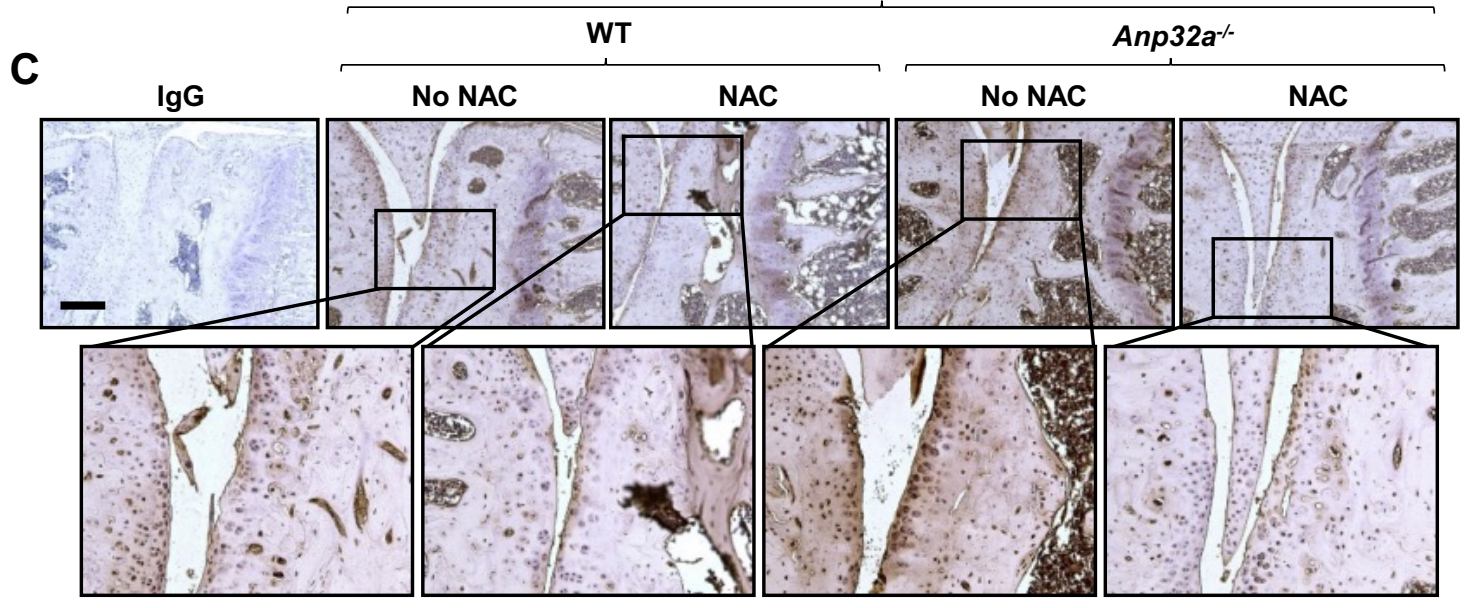

B

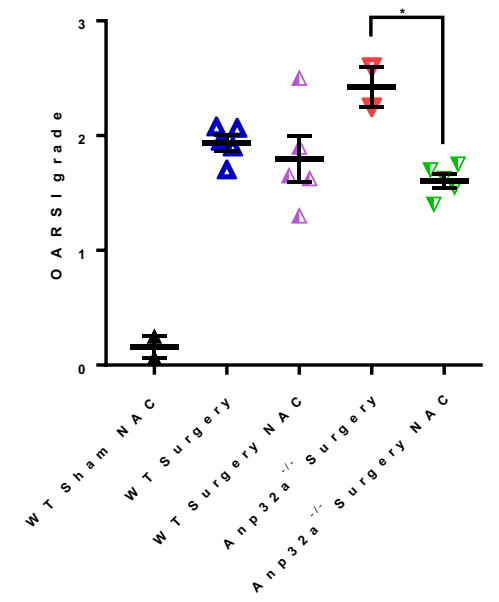

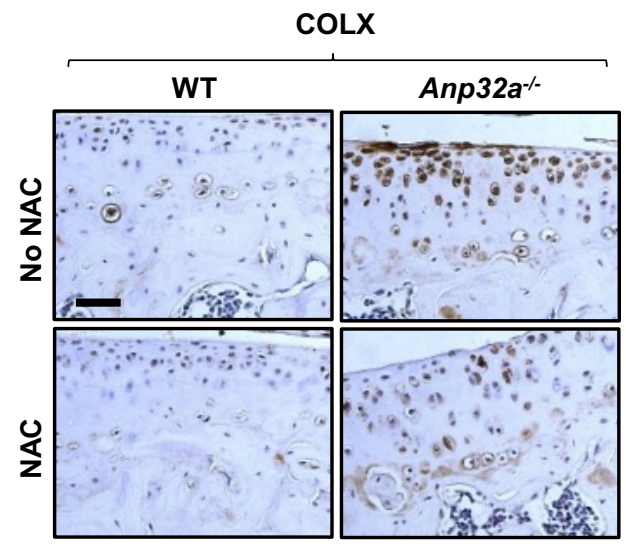

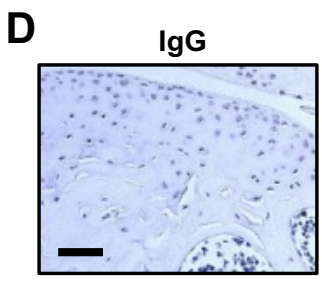

E

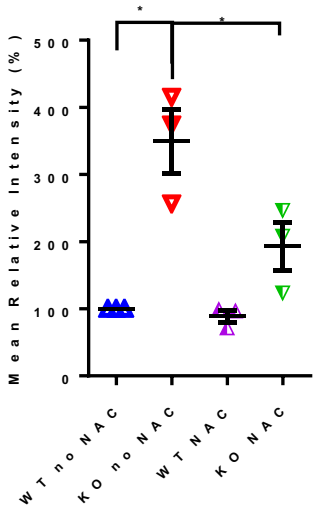


Fig. 4. Antioxidant treatment prevents osteoarthritis in Anp32a-deficient mice. (A) Hematoxylin-SafraninO staining of WT Sham-operated mice, and WT and Anp32a mice 12 weeks after induction of osteoarthritis by the DMM model, treated with vehicle or N-acetylcysteine (NAC). Scale bar, $200 \mu \mathrm{m}$. (B) Quantification of cartilage damage in the knee joints from Anp32a $a^{-/}$mice and WT mice after DMM and NAC treatment, by OARSI severity grade score $(* P$ $<0.05$, one-way ANOVA and Bonferroni corrected post test; $\mathrm{n}=2$ (WT-Sham NAC), 5 (WTSurgery), 5 (WT-Surgery NAC), 2 (Anp32a $a^{-/-}$-Surgery), 5 (Anp32a $a^{-/-}$-Surgery NAC). (C) ROS levels assessed by immunohistochemical detection of 8-OHdG in adult knees from WT and Anp32 $a^{-/}$mice after induction of DMM osteoarthritis and treated or not with NAC. Scale bar, 200 $\mu \mathrm{m}$. (D) Immunohistochemistry showing increased type X collagen levels in the articular cartilage of Anp32a $a^{-/}$compared to WT mice, that is countered by NAC treatment. (E) Quantification of type X collagen staining in the articular cartilage from Anp32a $a^{-/-}$mice compared to WT mice, with or without NAC treatment, by digital image analysis $(* P<0.05$, one-way ANOVA and Bonferroni corrected post test; $n=3$ ). The images are representative of images from 3 to 5 different mice. Scale bar, $200 \mu \mathrm{m}(\mathrm{A}, \mathrm{C})$ and $50 \mu \mathrm{m}(\mathrm{D})$. Error bars indicate mean \pm s.e.m. 
Figure 5

A
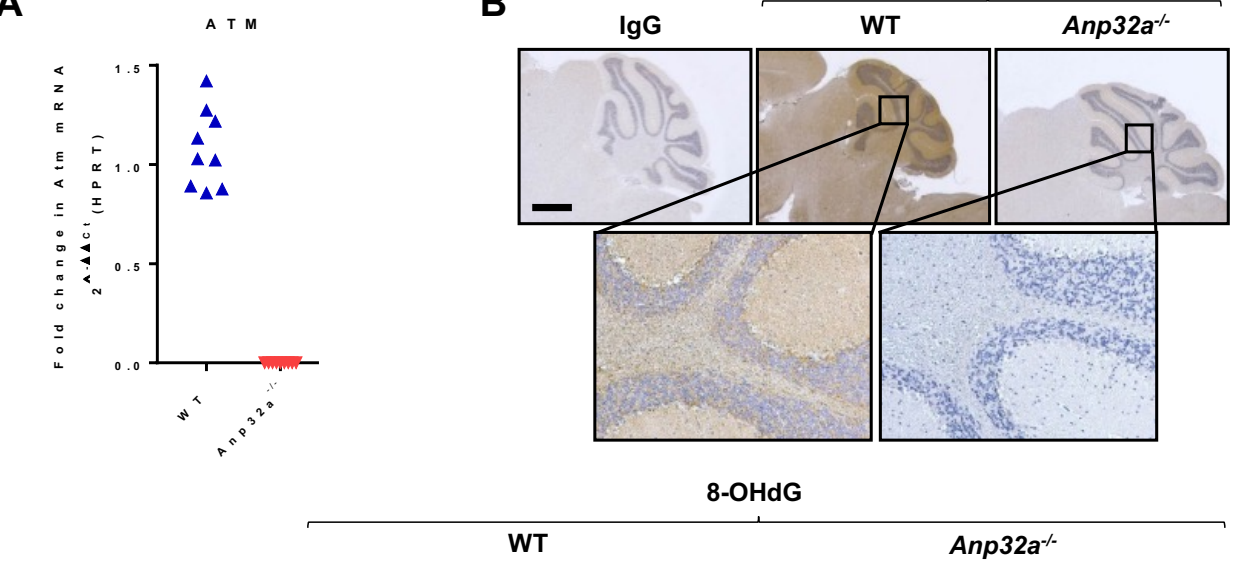

C

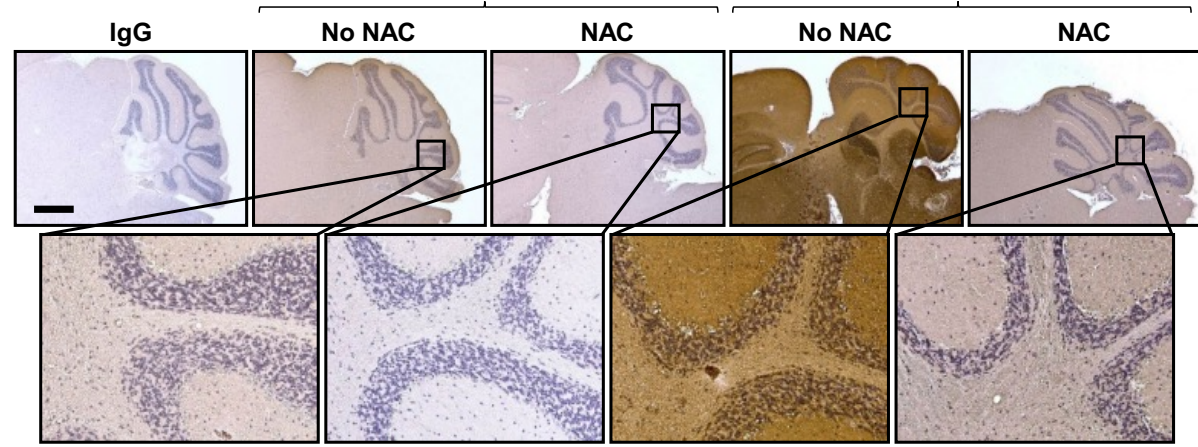

D

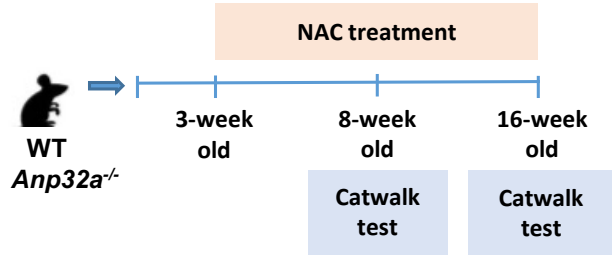

E
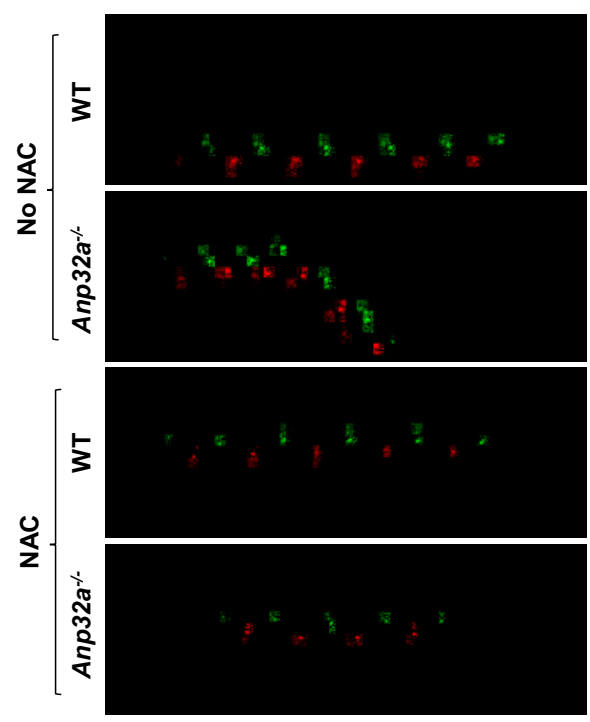

F

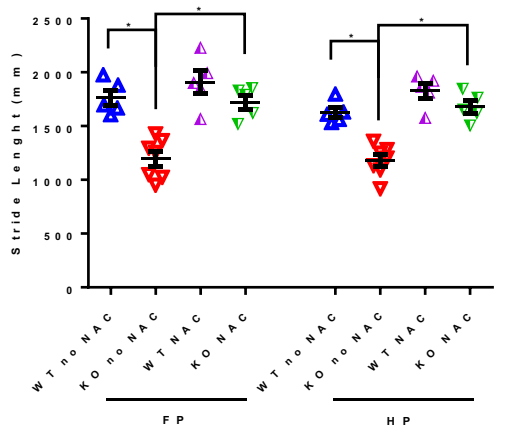

G

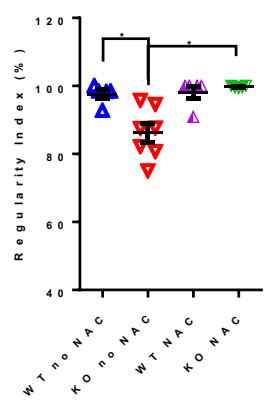


Fig. 5. Anp32a deficiency leads to neurological defects related to ataxia that are prevented by antioxidant treatment. (A) $A T M$ expression measured by quantitative PCR, in adult brain from 8-week old WT and Anp32a-deficient mice (n=9). (B) Immunohistochemistry showing reduced ATM levels in 8-week old cerebellum from Anp32a-deficient mice compared to WT mice. Scale bar, $500 \mu \mathrm{m}$. (C) ROS levels assessed by immunohistochemical detection of 8-OHdG in 8week old cerebellum from WT and Anp32a-deficient mice. Scale bar, $500 \mu \mathrm{m}$. (D) Time course of oral treatment with NAC and Catwalk tests in WT and Anp32a-deficient mice. (E) Gait analysis of 8-week old WT and Anp32a-deficient mice treated with NAC, assessed by the CatWalk automated gait analysis system. Footprint colors were assigned manually (green, right; red, left; light print, forelimbs; dark print, hindlimbs) and parameters calculated by the software. (F) The average stride length was shorter for Anp32a-deficient mice than for WT mice in both front-paw (FP) and hind-paw (HP), a defect that was rescued by NAC treatment. (G) 8-week old WT mice had more consistent walking patterns, as revealed by a higher regularity index compared to 8-week old Anp32a-deficient mice. This defect was rescued by NAC treatment. ( ${ }^{*} \mathrm{P}<0.001$ for $\mathrm{F}, * \mathrm{P}<0.01$ for G, one-way ANOVA and Bonferroni corrected post test; $\mathrm{n}=5$ (WT no NAC), 7 (KO no NAC), 5 (WT NAC), 5 (KO NAC). The images are representative of images from $3(\mathbf{B}, \mathbf{C})$ or 5 to 7 (E) different mice. Error bars indicate mean \pm s.e.m. 
Figure 6

A

B

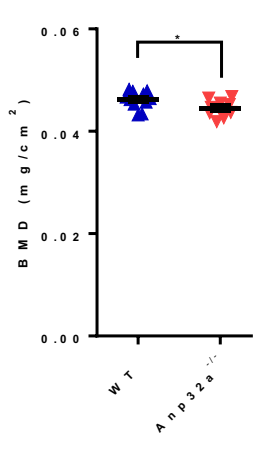

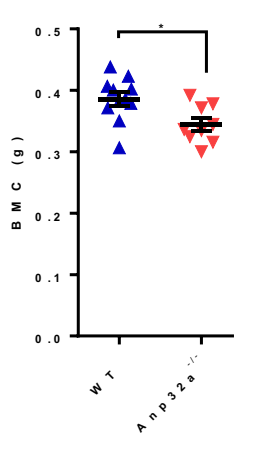

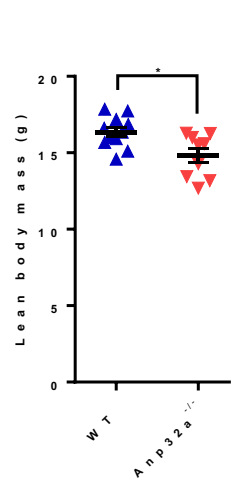

C
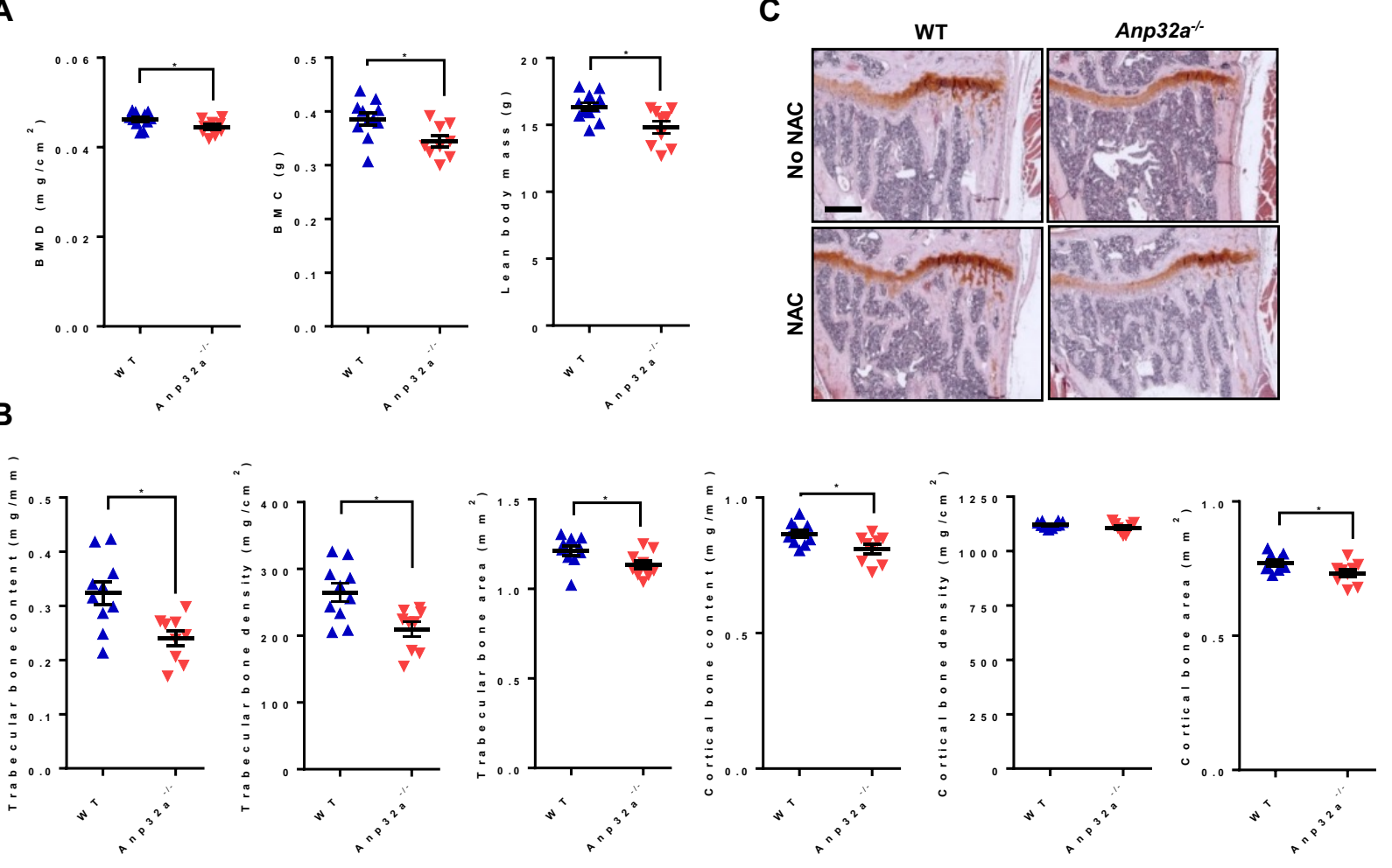

D
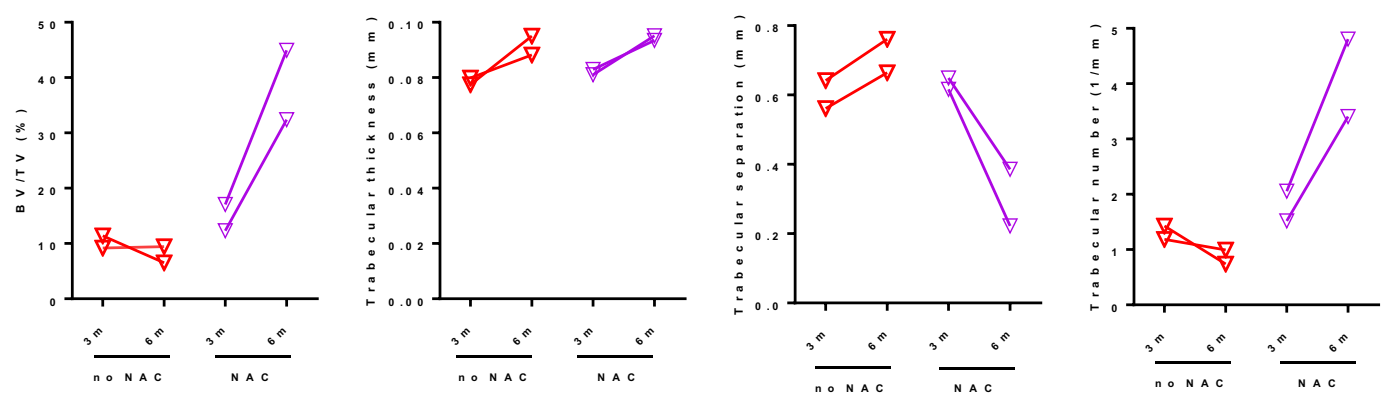

E

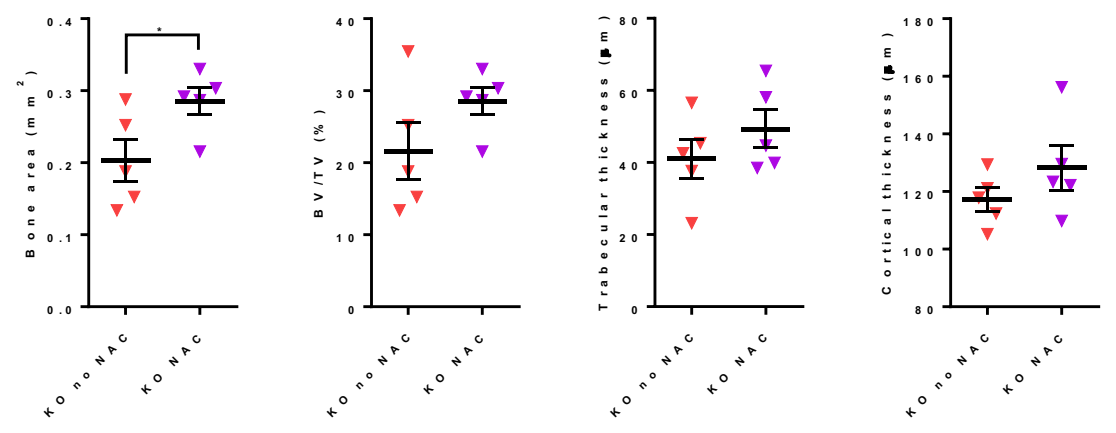


Fig. 6. Anp32a deficiency leads to osteopenia that is responsive to antioxidant treatment. (A) Subcapital dual energy X-ray absorptiometry (DEXA) analysis of bone mineral density (BMD), bone mineral content (BMC) and lean body mass in 12-week old female Anp32a-/ mice compared to WT littermates $(* P<0.05, t$-test; $\mathrm{n}=11(\mathrm{WT})$ and $9(\mathrm{KO}))$. (B) Peripheral quantitative computed tomography analysis (pQCT) of trabecular and cortical bone parameters in femora from 12-week old female Anp32a $a^{-/-}$mice and WT littermates (* $P<0.05, t$-test; $\mathrm{n}=10$ (WT) and 9 (KO)). (C) Hematoxylin-SafraninO staining of dissected tibia from 16-week old male Anp32a $a^{-/}$ mice compared to $\mathrm{C} 57 \mathrm{Bl} / 6 \mathrm{WT}$ controls with or without NAC treatment for 13 weeks $(* P<0.05$, $t$-test; $\mathrm{n}=5$ ). Scale bar $250 \mu \mathrm{m}$. (D) In vivo micro-computed tomography ( $\mu \mathrm{CT}$ ) of tibiae from female Anp $32 a^{-/-}$mice treated or not with NAC for 12 weeks from the age of 3 months until the age of 6 months (BV/TV bone volume/tissue volume) $(n=2)$. (E) Histomorphometry analysis of tibiae from male Anp32a $a^{-/-}$mice treated or not with NAC for 13 weeks from the age of 3 weeks until the age of 16 weeks $(* P<0.05, t$-test; $\mathrm{n}=5$ ). 
Supplementary Materials:

SUPPLEMENTARY FIGURES

Supplementary Figure 1

A

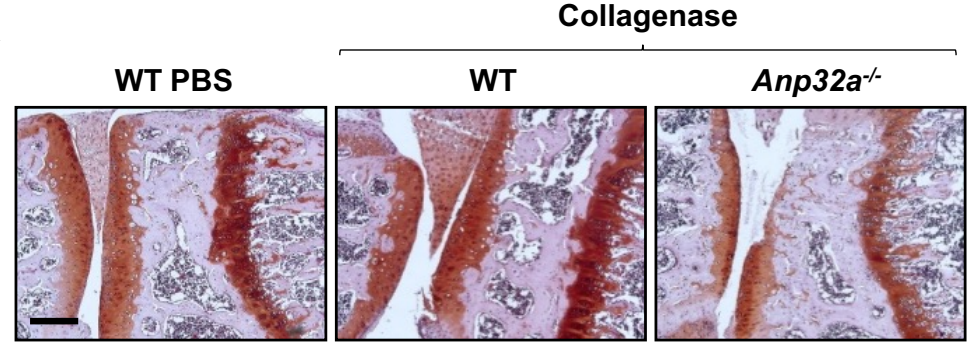

C

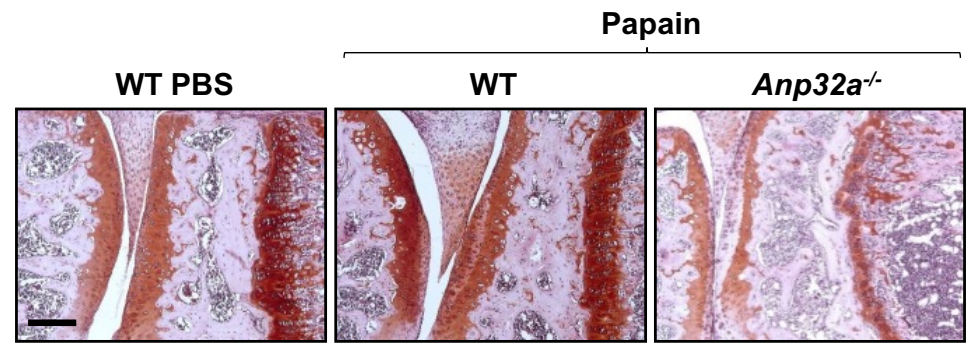

B

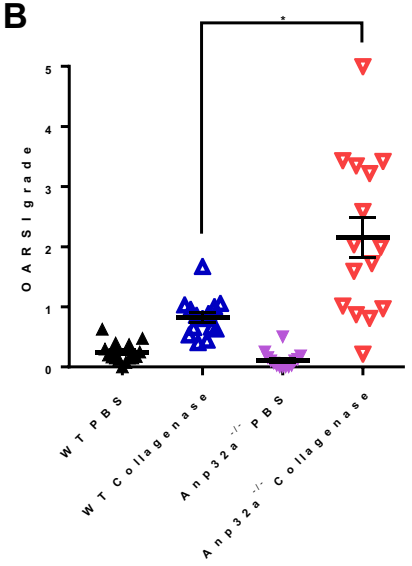

D

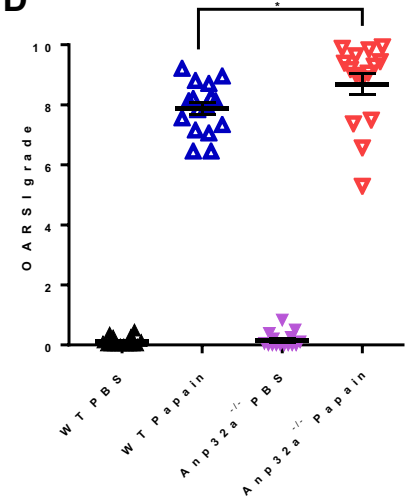

Fig. S1. Loss of ANP32A increases the severity of osteoarthritis in the collagenase- and papain-induced mouse models. (A) Hematoxylin-SafraninO staining of WT and Anp32 $a^{-/-}$mice injected with collagenase. (B) Comparison of knee joints from WT and Anp32a $a^{-/}$mice injected with collagenase, by OARSI severity grade score $(* P<0.001$, one-way ANOVA and Bonferroni corrected post test; $\mathrm{n}=15)$. (C) Hematoxylin-SafraninO staining of WT and Anp32a $a^{-/-}$mice injected with papain. (D) Comparison of knee joints from WT and Anp32a $a^{-/}$mice injected with papain, by OARSI severity grade score $(* P<0.05$, one-way ANOVA and Bonferroni corrected post test; $n=15)$. The images are representative of images from 15 different mice. Scale bars, 200 $\mu \mathrm{m}$. Error bars indicate mean \pm s.e.m. 


\section{Supplementary Figure 2}

A
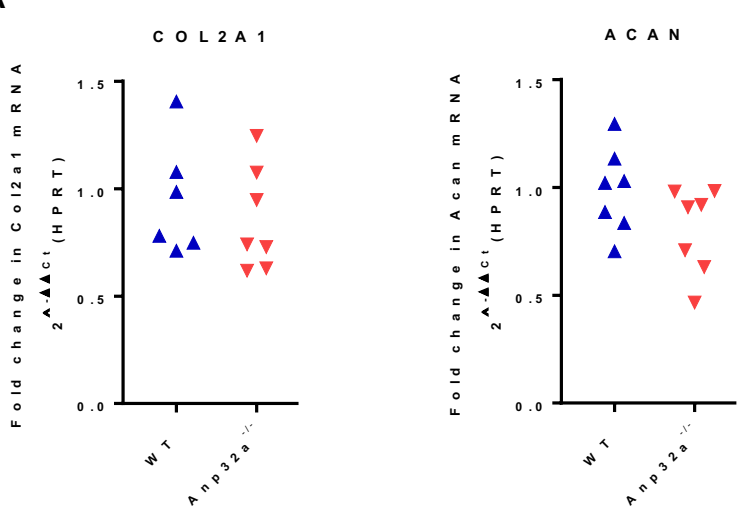

B

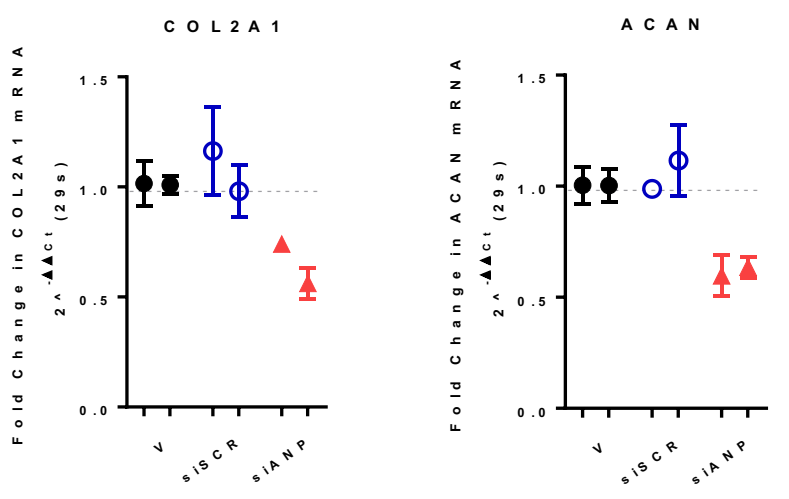

Fig. S2. Expression of molecular markers associated with the healthy chondrocyte in the presence or absence of ANP32A. . Expression levels of healthy articular chondrocyte markers collagen 2 (COL2A1) or aggrecan (ACAN) measured by quantitative PCR in (A) tibial articular cartilage of 8-week old male Anp32a-deficient mice and WT mice ( $\mathrm{n}=6$ to 7 per group) and in (B) human healthy articular chondrocytes with siRNA mediated silencing of ANP32A. Data are from one experiment with three technical replicates per mouse (A) and from two biologically different experiments each with three technical replicates (B). 


\section{Supplementary Figure 3}
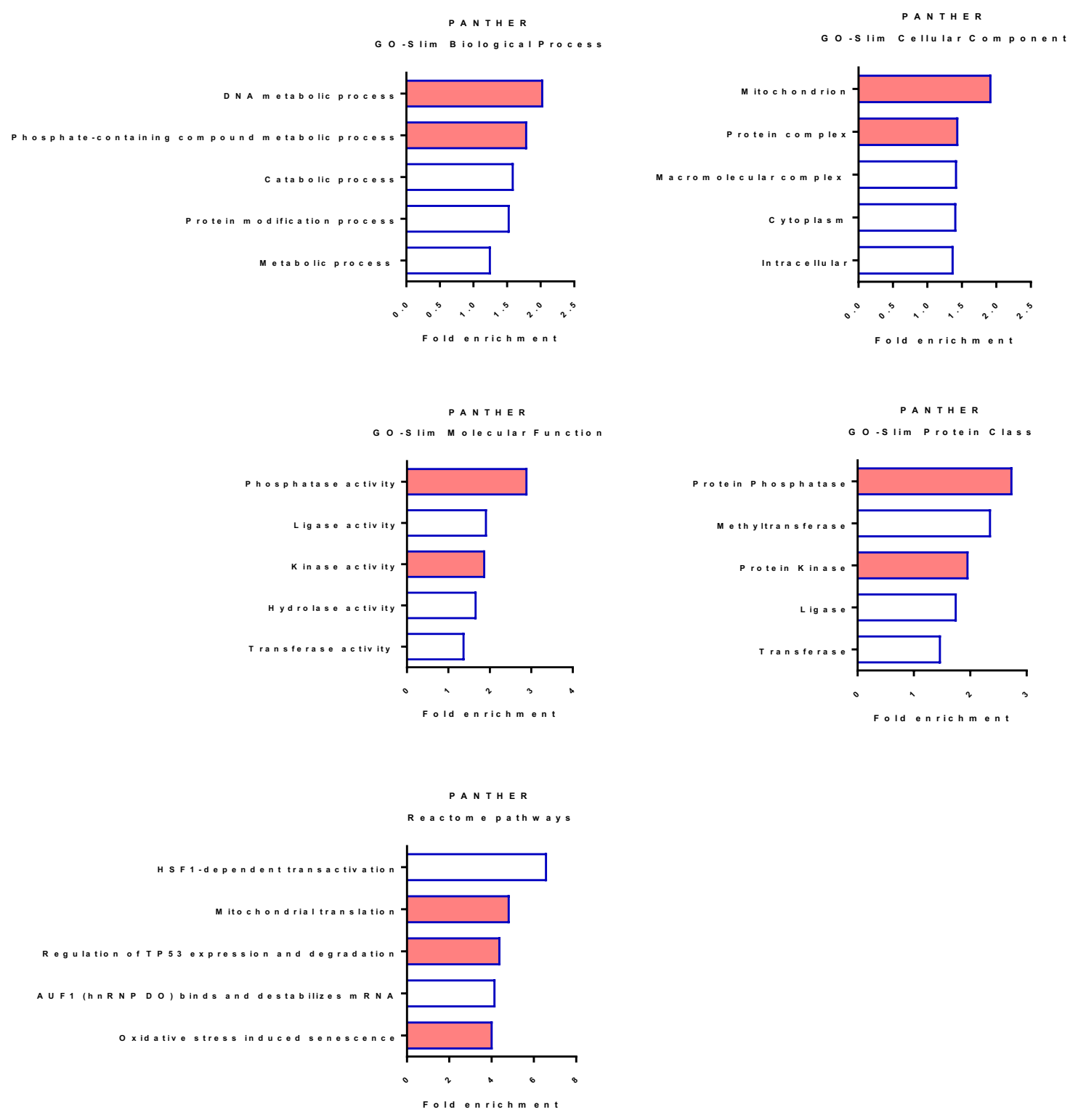

Fig. S3. Transcriptome network analysis of articular cartilage of Anp32a-deficient mice. Transcriptome analysis of reactome pathways, top biological processes, molecular functions, cellular components and protein classes altered in the articular cartilage of 8 -week old male Anp32a-deficient mice compared to wild-type mice, using the "GO-slim" and Protein class ontology of the PANTHER Database. Pathways and processes of particular interest for osteoarthritis are highlighted. 


\section{Supplementary Figure 4}

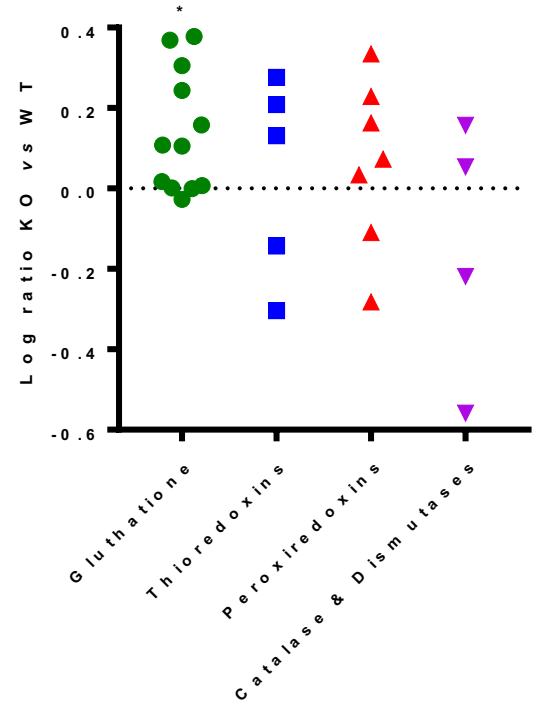

Fig. S4. Compensatory regulation of anti-oxidant systems in the articular cartilage of Anp32a-deficient mice. Transcriptome data from the articular cartilage of 8-week old male Anp32a-deficient mice (KO) and wild-type (WT) mice were queried for changes in expression of genes associated with the main antioxidant systems of the cell (for individual genes see Supplementary Table 3). Genes involved in synthesis, oxidation and reduction of glutathione were upregulated in Anp32a-deficient mice compared to WT animals $\left({ }^{*} P<0.01\right.$, one-group $t$-test against hypothetical mean 0 for Log-ratio KO vs WT). 


\section{Supplementary Figure 5}
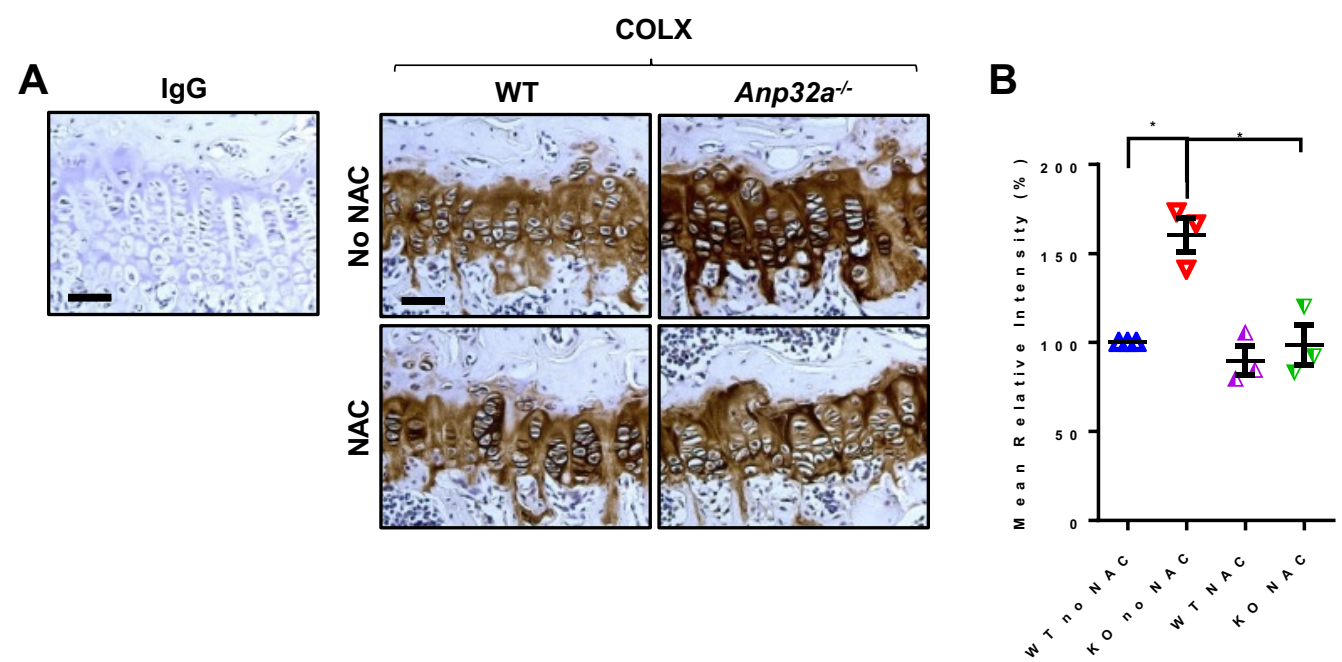

Fig. S5. Immunohistochemical detection of type $X$ collagen levels in the growth plates of Anp32a $a^{-/}$compared to WT mice. (A) Immunohistochemistry showing increased type X collagen levels in the growth plates of Anp $32 a^{-/}$compared to WT mice, that is countered by NAC treatment. (B) Quantification of type X collagen staining in the growth plate from Anp32a $a^{-/}$mice compared to WT mice, with or without NAC treatment, by digital image analysis $(* P<0.01$, one-way ANOVA and Bonferroni corrected post test; $n=3$ ). The images are representative of images from 3 different male mice. Scale bar, $50 \mu \mathrm{m}$. Error bars indicate mean \pm s.e.m. 


\section{Supplementary Figure 6}

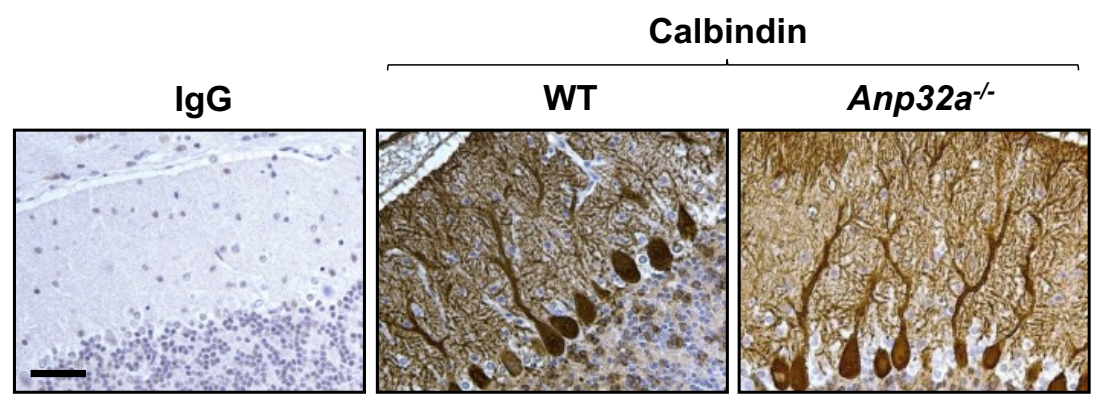

Fig. S6. Calbindin immunostaining of cerebellar Purkinje Cells of 16-week old WT and Anp32a-deficient mice. The images are representative of images from 3 different male mice. Scale bar, $50 \mu \mathrm{m}$. 


\section{Supplementary Figure 7}

A

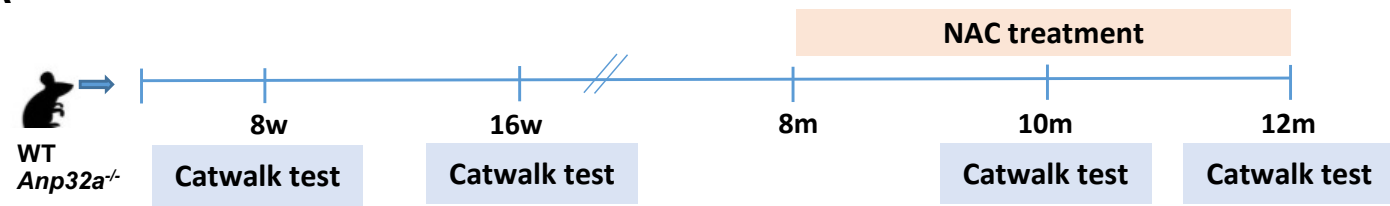

B
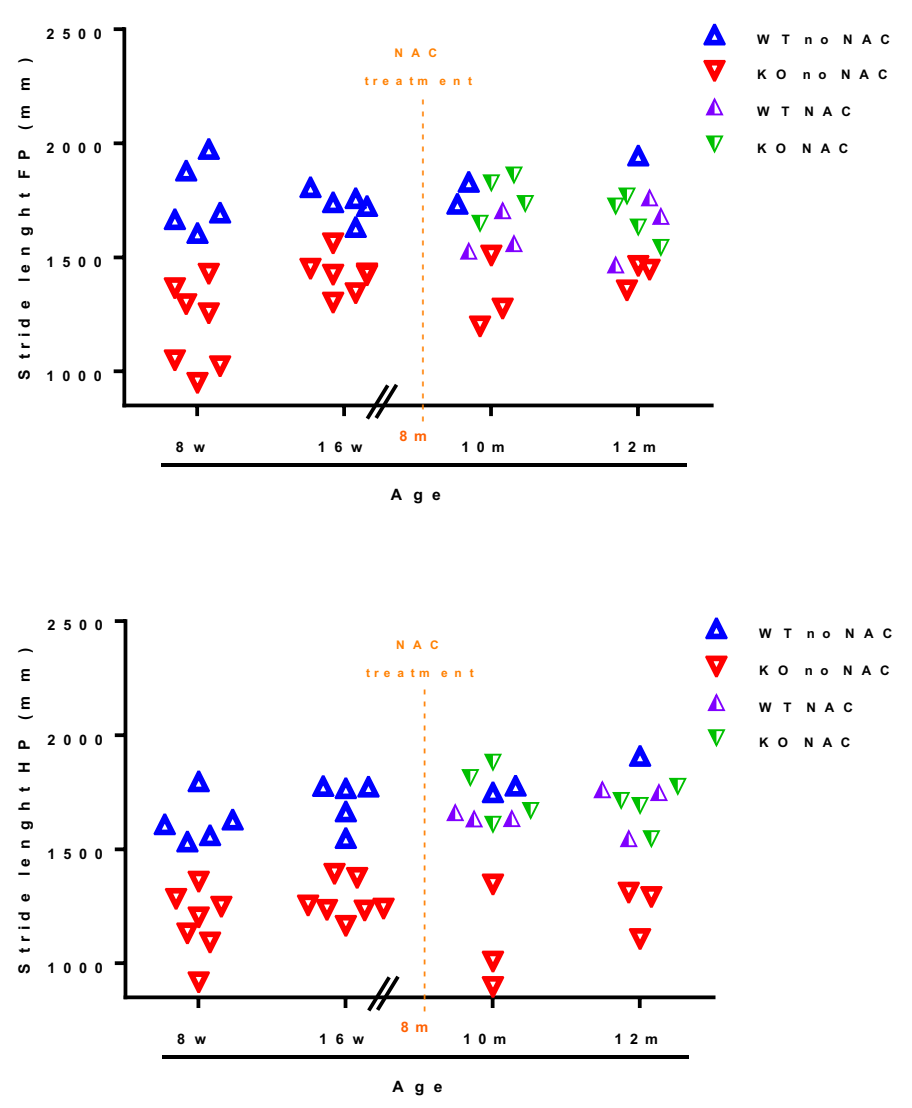

C

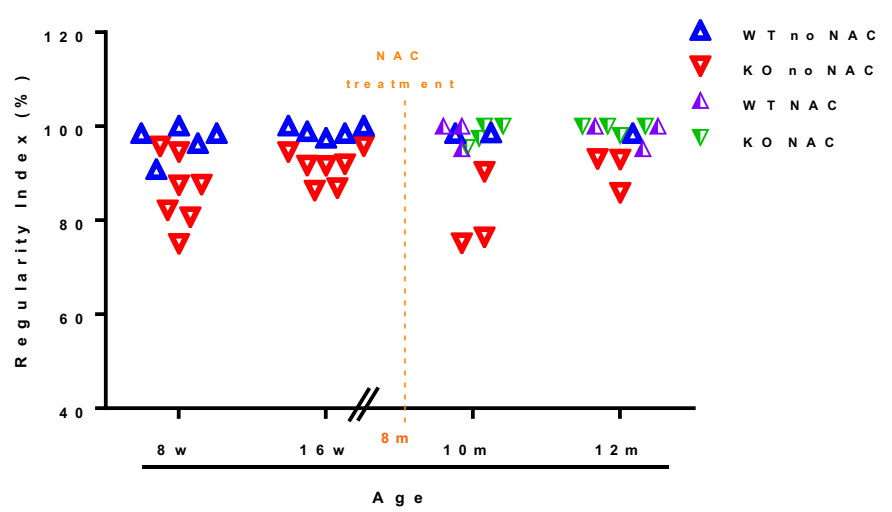


Fig. S7. Late stage antioxidant intervention in Anp32a-deficient mice ameliorates ataxiarelated defects. (A) Scheme of time course of oral treatment with NAC and Catwalk tests in WT and Anp32a-deficient mice. (B) The reduction in average stride length in Anp32a-deficient male mice compared to WT mice was restored upon NAC treatment from 8- to 10- month old, in both front-paw (FP) and hind-paw (HP) $(P<0.01$ for KO NAC vs. KO NO NAC, $P<0.001$ for WT $v s$. KO NO NAC, 2-way ANOVA with interaction between genotype and time $(P<0.001)$, effect of time $(P<0.01)$ and effect of genotype $(P<0.0001))$. (C) Regularity index was restored in Anp32adeficient mice upon treatment with NAC $(P<0.01$ for KO NAC $v s$. KO NO NAC and for WT $v s$. KO NO NAC, 2-way ANOVA with interaction between genotype and time $(P<0.05)$, effect of time $(P<0.05)$ and effect of genotype $(P<0.001))$. 


\section{Supplementary Figure 8}

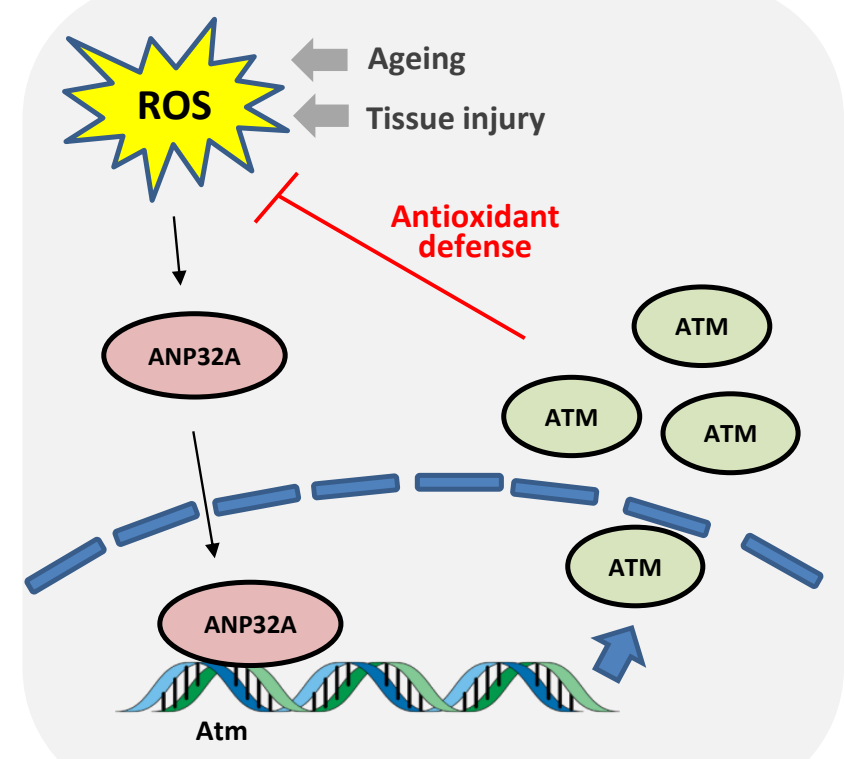

Fig. S8. Model for the role of ANP32A on oxidative stress. Upon increase in ROS levels, resulting from tissue stress or the process of ageing, ANP32A translocates to the nucleus and binds to the Atm gene, triggering its expression as a protective mechanism to enhance the antioxidant capacities of the cell. 


\section{SUPPLEMENTARY TABLES}

\section{Supplementary Table 1. Patient characteristics.}

\begin{tabular}{|c|c|c|c|}
\hline \multirow{2}{*}{ Group } & Patient nr. & Age & Gender \\
\hline Ex vivo and in vitro experiments \\
\hline Non-osteoarthritis & 1 & 72 & Male \\
\hline \multirow{6}{*}{} & 2 & 77 & Male \\
\cline { 2 - 4 } & 3 & 76 & Female \\
\cline { 2 - 4 } & 4 & 86 & Female \\
\cline { 2 - 4 } & 5 & 76 & Male \\
\cline { 2 - 4 } & 6 & 74 & Female \\
\cline { 2 - 4 } & 7 & 62 & Female \\
\cline { 2 - 4 } & 8 & 75 & Female \\
\hline Osteoarthritis & 9 & 73 & Female \\
\hline \multirow{6}{*}{} & 10 & 73 & Female \\
\cline { 2 - 4 } & 11 & 64 & Female \\
\cline { 2 - 4 } & 12 & 74 & Female \\
\cline { 2 - 4 } & 13 & 84 & Female \\
\cline { 2 - 4 } & 14 & 74 & Female \\
\cline { 2 - 4 } & 15 & 84 & Female \\
\cline { 2 - 4 } & 16 & 70 & Male \\
\cline { 2 - 4 } & 17 & 53 & Female \\
\hline 18 & 47 & Female \\
\hline
\end{tabular}




\section{Supplementary Table 2. Top ranked genes of transcriptome network of articular cartilage}

\section{of Anp32a-deficient mice.}

\begin{tabular}{|c|c|c|c|}
\hline $\begin{array}{c}\text { Gene } \\
\text { symbol }\end{array}$ & Gene title & $\begin{array}{c}\text { Log ratio: } \\
A n p 32 a^{-l-} \text { vs. WT }\end{array}$ & $P$-value \\
\hline \multicolumn{4}{|c|}{ Top down-regulated } \\
\hline Anp32a & Acidic (leucine-rich) nuclear phosphoprotein 32 family, member A & $-5,282008604$ & $1,75 \mathrm{E}-10$ \\
\hline Ppcde & Phosphopantothenoylcysteine decarboxylase & $-2,44367331$ & $2,62 \mathrm{E}-10$ \\
\hline Atm & Ataxia telangiectasia mutated & $-1,664095956$ & $8,33 \mathrm{E}-08$ \\
\hline Pclaf & PCNA clamp associated factor & $-3,054429474$ & $1,10 \mathrm{E}-07$ \\
\hline Plet1os & Placenta expressed transcript 1 , opposite strand & $-2,38741279$ & $3,22 \mathrm{E}-07$ \\
\hline Ren2 & Reticulocalbin 2 & $-1,336037559$ & $3,19 \mathrm{E}-06$ \\
\hline Nxpe4 & Neurexophilin and PC-esterase domain family, member 4 & $-1,006861361$ & $4,69 \mathrm{E}-06$ \\
\hline Eda2r & Ectodysplasin A2 receptor & $-1,084627442$ & $8,93 \mathrm{E}-05$ \\
\hline Ociad2 & OCIA domain containing 2 & $-1,13120499$ & 0,000122 \\
\hline Lrp2bp & Lrp2 binding protein & $-1,116254296$ & 0,00015 \\
\hline \multicolumn{4}{|c|}{ Top up-regulated } \\
\hline Mid1 & Midline 1 & 1,473476787 & $1,75 \mathrm{E}-06$ \\
\hline Ighg & Immunoglobulin heavy chain (gamma polypeptide) & 2,760380518 & $5,24 \mathrm{E}-06$ \\
\hline Igh-VJ558 & Immunoglobulin heavy chain (J558 family) & 1,404989959 & $7,70 \mathrm{E}-05$ \\
\hline Jchain & Immunoglobulin joining chain & 1,698052461 & 0,000108247 \\
\hline Lrrc49 & Leucine rich repeat containing 49 & 1,123443713 & 0,000154359 \\
\hline Ighm & Immunoglobulin heavy constant mu & 1,725390275 & 0,000322652 \\
\hline Iglv1 & Immunoglobulin lambda variable 1 & 1,046587665 & 0,000444521 \\
\hline $\mathrm{Hmbs}$ & Hydroxymethylbilane synthase & 1,399103193 & 0,000720047 \\
\hline Igk & Immunoglobulin kappa chain complex & 1,241928459 & 0,000945658 \\
\hline Bex 4 & Brain expressed X-linked 4 & 1,089494925 & 0,002740911 \\
\hline
\end{tabular}


Supplementary Table 3. Gene expression of main antioxidant in transcriptome network of articular cartilage of Anp32a-deficient mice.

\begin{tabular}{|c|c|c|c|}
\hline Gene symbol & Gene title & $\begin{array}{c}\text { Log ratio: } \\
\text { Anp32a }^{-1-} \text { vs. WT }\end{array}$ & $P$-value \\
\hline \multicolumn{4}{|c|}{ Gluthation regulation } \\
\hline Gclc & Glutamate-cysteine ligase, catalytic subunit & 0,37830635 & 0,02736474 \\
\hline Gclm & Glutamate-cysteine ligase, modifier subunit & 0,30548062 & 0,17796085 \\
\hline Gss & Glutathione synthetase & 0,00724726 & 0,91603549 \\
\hline Gsr & Glutathione reductase & 0,24402975 & 0,19908575 \\
\hline Gpx1 & Glutathione peroxidase 1 & 0,36911058 & 0,01123824 \\
\hline $\mathrm{Gpx} 2$ & Glutathione peroxidase 2 & 0,10776233 & 0,24914101 \\
\hline Gpx3 & Glutathione peroxidase 3 & 0,0015783 & 0,98271166 \\
\hline Gpx4 & Glutathione peroxidase 4 & $-0,0271767$ & 0,77878603 \\
\hline Gpx5 & Glutathione peroxidase 5 & $-0,0002899$ & 0,99670251 \\
\hline Gpx6 & Glutathione peroxidase 6 & 0,15751416 & 0,11917126 \\
\hline Gpx7 & Glutathione peroxidase 7 & 0,01733939 & 0,90626585 \\
\hline $\mathrm{Gpx} 8$ & Glutathione peroxidase 8 (putative) & 0,10563988 & 0,50892184 \\
\hline \multicolumn{4}{|c|}{ Thioredoxins } \\
\hline Txn1 & Thioredoxin 1 & 0,20896773 & 0,07142316 \\
\hline $\operatorname{Txn} 2$ & Thioredoxin 2 & $-0,3040016$ & 0,02184689 \\
\hline Txnrd1 & Thioredoxin reductase 1 & $-0,1425567$ & 0,02867556 \\
\hline Txnrd2 & Thioredoxin reductase 2 & 0,27591502 & 0,04936511 \\
\hline Txnrd3 & Thioredoxin reductase 3 & 0,13142402 & 0,04730358 \\
\hline \multicolumn{4}{|c|}{ Peroxiredoxins } \\
\hline $\operatorname{Prdx} 1$ & Peroxiredoxin 1 & 0,03368836 & 0,62931903 \\
\hline $\operatorname{Prdx} 2$ & Peroxiredoxin 2 & 0,334464 & 0,04863448 \\
\hline Prdx3 & Peroxiredoxin 3 & $-0,281844$ & 0,06549478 \\
\hline $\operatorname{Prdx} 4$ & Peroxiredoxin 4 & 0,22857676 & 0,14892342 \\
\hline $\operatorname{Prdx} 5$ & Peroxiredoxin 5 & 0,0729794 & 0,38646309 \\
\hline $\operatorname{Prdx} 6$ & Peroxiredoxin 6 & 0,16314007 & 0,19724725 \\
\hline $\operatorname{Prdx} 6 \mathrm{~b}$ & Peroxiredoxin $6 \mathrm{~B}$ & $-0,109492$ & 0,22008571 \\
\hline \multicolumn{4}{|c|}{ Catalase and dismutases } \\
\hline Cat & Catalase & 0,15719095 & 0,16590453 \\
\hline Sod1 & Superoxide dismutase 1 , soluble & 0,05345555 & 0,36431195 \\
\hline Sod2 & Superoxide dismutase 2 , mitochondrial & $-0,5586937$ & 0,02815667 \\
\hline Sod3 & Superoxide dismutase 3 , extracellular & $-0,2190621$ & 0,13887811 \\
\hline
\end{tabular}


Supplementary Table 4. Gait parameters of early stage antioxidant intervention in Anp32adeficient mice.

A

\begin{tabular}{|c|c|c|c|c|c|c|c|c|}
\hline \multicolumn{9}{|c|}{8 weeks } \\
\hline $\begin{array}{l}\text { Gait parameter } \\
\text { (unit) }\end{array}$ & & Wildtype & Anp32a $a^{-/-}$ & Wildtype + NAC & Anp $32 a^{-/ 2}+\mathrm{NAC}$ & $\begin{array}{l}\text { ANOVA } \\
\text { (P-value) }\end{array}$ & Post tests & $P$-value \\
\hline \multirow{4}{*}{$\begin{array}{l}\text { Intensity } \\
(0-255)\end{array}$} & Front paws & $27.71+0.803$ & $22.24+1.094$ & $23.73+0.900$ & $19.52+0.611$ & 0.0001 & Wildtype vs. $A n p 32 a^{-/}$ & 0.0266 \\
\hline & & & & & & & Anp $32 a^{-/ /}$vs.Anp $32 a^{-/ /}+\mathrm{NAC}$ & 0.0171 \\
\hline & Hind paws & $40.88+1.124$ & $31.49+2.099$ & $36.33+1.26$ & $25.31+0.8567$ & 0.0001 & Wildtype vs. Anp $32 a^{-/ /}$ & 0.1778 \\
\hline & & & & & & & Anp $32 a^{-/ /}$vs. Anp $32 a^{-/ /}+\mathrm{NAC}$ & 0.0001 \\
\hline \multirow{4}{*}{$\begin{array}{l}\text { Stand } \\
(s)\end{array}$} & Front paws & $0.1673+0.122$ & $0.1617+0.014$ & $0.2295+0.018$ & $0.1233+0.006$ & 0.0004 & Wildtype vs. Anp $32 a^{-/}$ & 0.0345 \\
\hline & & & & & & & Anp $32 a^{-/ /}$vs. Anp $32 a^{-/}+\mathrm{NAC}$ & 0.0003 \\
\hline & Hind paws & $0.1693+0.006$ & $0.176+0.007$ & $0.2643+0.256$ & $0.1347+0.007$ & 0.0002 & Wildtype vs. Anp $32 a^{-/-}$ & 0.0055 \\
\hline & & & & & & & Anp $32 a^{-/ /}$vs. Anp $32 a^{-/}+\mathrm{NAC}$ & 0.0002 \\
\hline \multirow{4}{*}{$\begin{array}{l}\text { Duty cycle } \\
\text { (\% stand) }\end{array}$} & Front paws & $54.16+1.79$ & $50.23+2.503$ & $58.61+1.321$ & $44.86+1.695$ & 0.002 & Wildtype vs. Anp $32 a^{-/}$ & 0.5307 \\
\hline & & & & & & & Anp $32 a^{-/ /}$vs. Anp $32 a^{-/ /}+\mathrm{NAC}$ & 0.0002 \\
\hline & Hind paws & $57.37+1.597$ & $55.81+2.033$ & $63.42+1.258$ & $50.13+2.056$ & 0.0002 & Wildtype vs. $A n p 32 a^{-/ /}$ & 0.1094 \\
\hline & & & & & & & Anp $32 a^{-/ /}$vs. Anp $32 a^{-/ /}+\mathrm{NAC}$ & 0.0001 \\
\hline \multirow{3}{*}{$\begin{array}{l}\text { Swing speed } \\
\text { (s) }\end{array}$} & Front paws & $14.42+0.577$ & $12.5+0.511$ & $9.29+0.6873$ & $12.51+0.6299$ & 0.0001 & Wildtype vs. Anp $32 a^{-/}$ & 0.0001 \\
\hline & & & & & & & Anp $32 a^{-/ /}$vs. Anp $32 a^{-/ /}+\mathrm{NAC}$ & 0.0096 \\
\hline & Hind paws & $15.38+1.096$ & $15.14+0.898$ & $12.53+0.733$ & $14.21+0.727$ & 0.0904 & & \\
\hline \multirow{4}{*}{$\begin{array}{l}\text { Base of support } \\
\text { (mm) }\end{array}$} & Front paws & $1776+121$ & $1798+36.2$ & $1596+160.9$ & $2030+87.73$ & 0.1339 & & \\
\hline & & & & & & & & \\
\hline & Hind paws & $2946+98.43$ & $3311+36.79$ & $3402+74.07$ & $3076+79.58$ & 0.0016 & Wildtype vs. Anp $32 a^{-/}$ & 0.0023 \\
\hline & & & & & & & Anp $32 a^{-/ /}$vs. Anp $32 a^{-/ /}+\mathrm{NAC}$ & 0.0365 \\
\hline
\end{tabular}


B

\begin{tabular}{|c|c|c|c|c|c|c|c|c|}
\hline \multicolumn{9}{|c|}{16 weeks } \\
\hline $\begin{array}{l}\text { Gait parameter } \\
\text { (unit) }\end{array}$ & & Wildtype & Anp32a $a^{-/-}$ & Wildtype + NAC & Anp $32 a^{-/-}+\mathrm{NAC}$ & $\begin{array}{l}\text { ANOVA } \\
\text { (P-ralue) }\end{array}$ & Post tests & $P$-value \\
\hline \multirow{4}{*}{$\begin{array}{l}\text { Intensity } \\
(0-255)\end{array}$} & Front paws & $28.95+0.5629$ & $31.1+1.549$ & $23.24+0.602$ & $24.35+2.256$ & 0.0027 & Wildtype vs. $A n p 32 a^{-/-}$ & 0.3295 \\
\hline & & & & & & & Anp $32 a^{-/}$vs. Anp $32 a^{-/ /}+\mathrm{NAC}$ & 1 \\
\hline & Hind paws & $38.59+1.163$ & $46.1+2.305$ & $32.96+0.907$ & $33.01+4.774$ & 0.0051 & Wildtype vs. Anp $32 a^{-/-}$ & 0.6833 \\
\hline & & & & & & & Anp $32 a^{-/} v s . A n p 32 a^{-/}+\mathrm{NAC}$ & 1 \\
\hline \multirow{4}{*}{$\begin{array}{l}\text { Stand } \\
(s)\end{array}$} & Front paws & $0.168+0.007$ & $0.189+0.017$ & $0.2652+0.011$ & $0.148+0.019$ & 0.0004 & Wildtype vs. $A n p 32 a^{-/-}$ & 0.0006 \\
\hline & & & & & & & Anp $32 a^{-/}$vs.Anp $32 a^{-/ 2}+\mathrm{NAC}$ & 0.0001 \\
\hline & Hind paws & $0.1833+0.015$ & $0.1913+0.016$ & $0.2871+0.014$ & $0.1567+0.019$ & 0.0001 & Wildtype vs. $A n p 32 a^{-/ 2}$ & 0.0013 \\
\hline & & & & & & & Anp $32 a^{-/ 2}$ vs.Anp $32 a^{-/ 2}+\mathrm{NAC}$ & 0.0001 \\
\hline \multirow{4}{*}{$\begin{array}{l}\text { Duty cycle } \\
\text { (\% stand) }\end{array}$} & Front paws & $52.16+0.4469$ & $56.51+2.013$ & $57.17+1.045$ & $48.56+3.331$ & 0.0155 & Wildtype vs. Anp $32 a^{-/-}$ & 0.4164 \\
\hline & & & & & & & Anp $32 a^{-/}$vs. Anp $32 a^{-/ 2}+\mathrm{NAC}$ & 0.0229 \\
\hline & Hind paws & $57.55+1.147$ & $57.92+1.142$ & $63.63+0.7256$ & $53.89+4.183$ & 0.0208 & Wildtype vs. Anp $32 a^{-/ /}$ & 0.2787 \\
\hline & & & & & & & Anp $32 a^{-1 / v}$ v. Anp $32 a^{-/ /}+\mathrm{NAC}$ & 0.0181 \\
\hline \multirow{4}{*}{$\begin{array}{l}\text { Swing speed } \\
\text { (s) }\end{array}$} & Front paws & $12.59+0.433$ & $13.62+1.203$ & $9.929+0.737$ & $13.49+1.129$ & 0.0221 & Wildtype vs. Anp $32 a^{-/-}$ & 0.2760 \\
\hline & & & & & & & Anp $32 a^{-/ /} v s . A n p 32 a^{-/ /}+\mathrm{NAC}$ & 0.0613 \\
\hline & Hind paws & $14.57+0.532$ & $15.34+1.326$ & $11.83+0.723$ & $16.41+1.427$ & 0.0218 & Wildtype vs. $A n p 32 a^{-/-}$ & 0.3970 \\
\hline & & & & & & & Anp $32 a^{-/}$vs.Anp $32 a^{-/ 2}+\mathrm{NAC}$ & 0.0257 \\
\hline \multirow{4}{*}{$\begin{array}{l}\text { Base of support } \\
(\mathrm{mm})\end{array}$} & Front paws & $1870+112.8$ & $1990+149.2$ & $1417+120.5$ & $1797+173$ & 0.0339 & Wildtype vs. Anp $32 a^{-/-}$ & 0.0001 \\
\hline & & & & & & & Anp $32 a^{-1 / v s . A n p} 32 a^{-/ 2}+\mathrm{NAC}$ & 0.0096 \\
\hline & Hind paws & $2972+83.84$ & $3196+96.16$ & $3582+226.5$ & $3241+223.3$ & 0.1513 & & \\
\hline & & & & & & & & \\
\hline
\end{tabular}


Supplementary Table 5. Primers used in qPCR analysis.

\begin{tabular}{|c|c|}
\hline Primer name & Sequence \\
\hline $\begin{array}{l}\text { hACAN-Fw } \\
\text { hACAN-Rv }\end{array}$ & $\begin{array}{l}\text { ACAAGGTCTCACTGCCCAAC } \\
\text { AATGGAACACGATGCCTGTC }\end{array}$ \\
\hline $\begin{array}{l}\text { hANP32A Fw } \\
\text { hANP32A Rv }\end{array}$ & $\begin{array}{l}\text { AGGACGCCCTCTGATGTGAA } \\
\text { CCCCTGAGACTCTGTTATCGC }\end{array}$ \\
\hline $\begin{array}{l}\text { hATM Fw } \\
\text { hATM Rv }\end{array}$ & $\begin{array}{l}\text { ATCGGCATTCAGATTCCAAA } \\
\text { TTTTCTGCCTGGAGGCTTGT }\end{array}$ \\
\hline $\begin{array}{l}\text { hCOL2A1-Fw } \\
\text { hCOL2A1-Rv }\end{array}$ & $\begin{array}{l}\text { GCACCTGCAGAGACCTGAAA } \\
\text { GTCTCGCCAGTCTCCATGTT }\end{array}$ \\
\hline $\begin{array}{l}\text { mACANFw } \\
\text { mACAN Rv }\end{array}$ & $\begin{array}{l}\text { GCTGCAGTGATCTCAGAAGAAG } \\
\text { GATGGTGAGGGAAGACCCTA }\end{array}$ \\
\hline $\begin{array}{l}\text { mANP32A Fw } \\
\text { mANP32A Rv }\end{array}$ & $\begin{array}{l}\text { ACGCCCTCTGATGTGAAAGA } \\
\text { CATCCGTGAGGCCTTCGATT }\end{array}$ \\
\hline $\begin{array}{l}\text { mATM Fw } \\
\text { mATM Rv }\end{array}$ & $\begin{array}{l}\text { TCTCCGATATGCCAGTCTTTTCA } \\
\text { ATACAGACTCAAGGCTGCCC }\end{array}$ \\
\hline $\begin{array}{l}\text { mCOL2A1 Fw } \\
\text { mCOL2A1 Rv }\end{array}$ & $\begin{array}{l}\text { CCAGGATGCCCGAAAATTAG } \\
\text { TTCTCCCTTGTCACCACGAT }\end{array}$ \\
\hline $\begin{array}{l}\text { hATM-3.5kb Fw } \\
\text { hATM-3.5kb Rv }\end{array}$ & $\begin{array}{l}\text { CCTTCTGTCGCTCTCTACTG } \\
\text { AATATGGCTGCTTCCTCCTG }\end{array}$ \\
\hline $\begin{array}{l}\text { hATM-1kb Fw } \\
\text { hATM-1kb Rv }\end{array}$ & $\begin{array}{l}\text { TGACCCACAAACAATCCCTCCTC } \\
\text { TTCTCCATCCTCCACGCAATACG }\end{array}$ \\
\hline $\begin{array}{l}\text { hATM-0.5kb Fw } \\
\text { hATM-0.5kb Rv }\end{array}$ & $\begin{array}{l}\text { AGGAACCACAATAAGGAACAAG } \\
\text { AAATTGCCGCGAGTTCAG }\end{array}$ \\
\hline $\begin{array}{l}\text { hATM+1kb Fw } \\
\text { hATM+1kb Rv }\end{array}$ & $\begin{array}{l}\text { GTGGATGATAATGTATGTGGTGATAGG } \\
\text { CCAAGGTAACACTGCGAGGTC }\end{array}$ \\
\hline
\end{tabular}




\section{ANP32A regulates ATM expression and prevents oxidative stress in cartilage, brain and bone}

Frederique M. F. Cornelis, Silvia Monteagudo, Laura-An K.A. Guns, Wouter den Hollander, Rob G.H.H. Nelissen, Lies Storms, Tine Peeters, Ilse Jonkers, Ingrid Meulenbelt and Rik J. Lories

\section{Supplementary document: Statistical Analysis}

\section{Introduction}

Statistical analyses were performed with R Studio (Version 1.0.15) and GraphPad Prism software. Parametric methods are generally preferred as they provide estimates and confidence intervals and generalize to more complex analyses. Thus, where possible, we worked with distribution-based parametric methods.

With regards to the normal distribution of data, we have consistently applied the following assumptions: (1) for many biological data such as gene expression data in tissue samples or in genetically identical animals, normal distribution was hypothesized and evaluated by $Q Q$ plots and Shapiro-Wilk normality tests, (2) homogeneity of variance was assessed by Levene's test. Depending on the outcome of this evaluation, we applied t-tests or ANOVAtests taking into account equal or different variances (applying Welch corrections). This approach provides the best power and is relatively robust against some degree of non-normal distribution. With these parametric tests, we also provide parameter estimates, something which the non-distribution based tests cannot. We also provide a sensitivity analysis for the different tests we performed mostly by applying the corresponding distribution-free test. Despite the reduced power of these tests such as Mann-Whitney tests or Kruskal-Wallis tests, all our key observations were confirmed.

When different groups were compared by ANOVA tests, pair-wise t-tests were subsequently performed applying a Bonferroni correction for multiple comparisons to control for Type I errors in rejecting the null hypothesis. In this accompanying document, biologically relevant comparisons are highlighted and linked to the null hypothesis for the experiment. 95\% confidence intervals of the difference between the means are reported where possible.

\section{Figure 1}

Fig-1A: Expression of ANP32A measured by quantitative PCR in articular chondrocytes from patients with hip osteoarthritis $(O A)(n=4)$ as compared to chondrocytes from hip fracture patients without $O A(n o n-O A)(n=4)$

Research question: are levels of ANP32A different between patients with and without osteoarthritis? Null hypothesis: no difference in expression levels of ANP32A between OA and non-OA groups.

Assumptions: independent groups - normal distribution with similar variances

1) Gene expression in tissue samples is commonly normally distributed

2) $\mathrm{QQ}$ (quantile-quantile) plots suggest normal distribution 

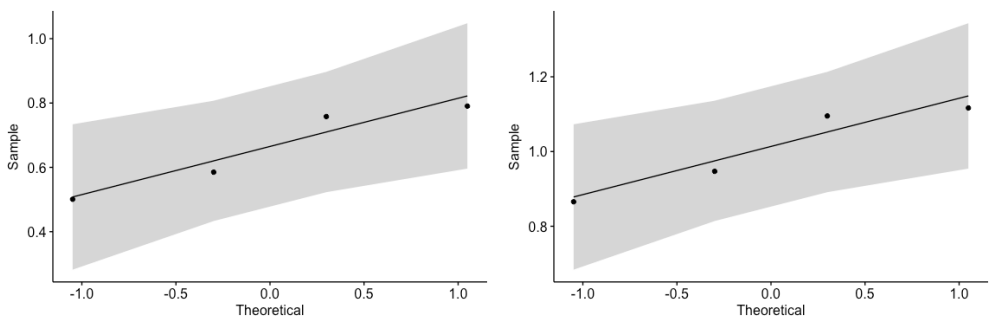

\begin{tabular}{|l|c|c|c|c|c|}
\hline \multicolumn{6}{|c|}{ Analysis selected: Two independent sample t-test (equal variance): } \\
\hline & $\begin{array}{c}\text { Shapiro-Wilk test } \\
\text { non-OA }\end{array}$ & $\begin{array}{c}\text { Shapiro-Wilk test } \\
\text { OA }\end{array}$ & Levene's test & p-value & $\begin{array}{c}\mathbf{9 5 \%} \mathrm{Cl} \text { of diff } \\
\text { of means }\end{array}$ \\
\hline OA vs & $\mathrm{W}=0.89585, \mathrm{p}-$ & $\mathrm{W}=0.9017, \mathrm{p}-$ & $\mathrm{F}$-value $=0.3247$ & $\mathrm{p}$-value $=$ & $\mathbf{0 . 1 2 3 1}$ to \\
NONOA & value $=0.4107$ & value $=0.4395$ & $\mathrm{p}$-value $=0.5895$ & $\mathbf{0 . 0 0 9 1}$ & $\mathbf{0 . 5 7 1 9}$ \\
\hline
\end{tabular}

Sensitivity analysis: Normal distribution not assumed: Mann-Whitney-test: $W=16, \mathbf{p}$-value $\mathbf{= 0 . 0 2 8 5 7}$

Fig-1D: Expression of ANP32A determined by RNA sequencing in paired preserved and damaged cartilage isolated from hips (o) and knees $(\Delta)$ from patients with osteoarthritis. The values for the preserved cartilage were set at 0 for each cartilage pair and data presented as log2-fold change (LOG2FC).

Research question: are levels of ANP32A different between preserved and damaged areas of cartilage in patients with osteoarthritis?

Null hypothesis: no difference in expression levels of ANP32A between preserved and damaged areas.

Data were analyzed as published before for the RAAK dataset [1], with differential gene expression being tested pairwise between preserved and lesioned samples followed by Benjamini-Hochberg False Discovery Rate correction.

Fig-1H: Quantification of articular cartilage damage in the knees of Anp32a ${ }^{-/}$mice (ANPKO) and WT mice knee joints after DMM by OARSI severity grade score

Research question: is the severity of articular cartilage damage (OARSI grade severity score) different between WT and ANPKO mice?

Null hypothesis: no difference in OARSI severity grade score between WT and ANPKO mice.

Assumptions: independent groups - normal distribution with unequal variances

1) model scores in genetically identical mice are commonly normally distributed

2) $\mathrm{QQ}$ (quantile-quantile) plots suggest normality.

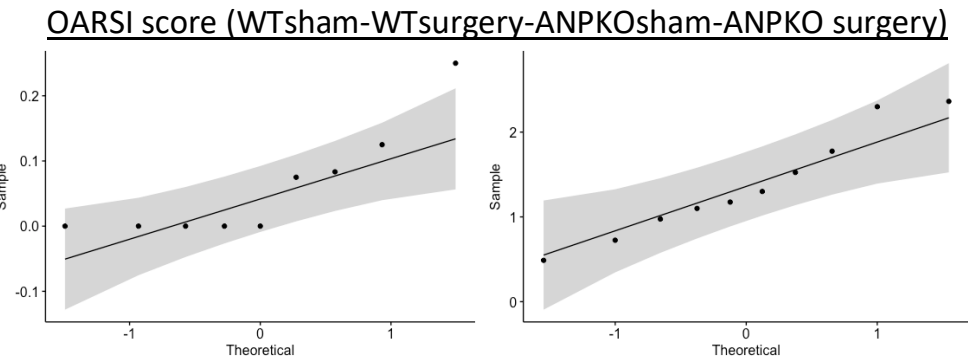



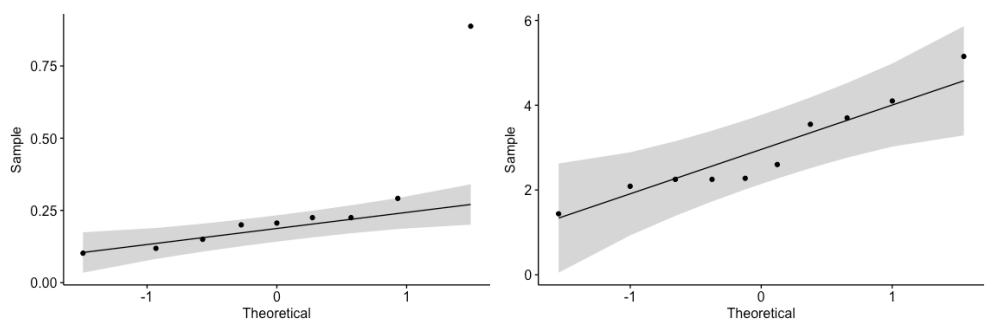

3) Shapiro-Wilk test: WTsham $W=0.75594$, $p$-value $=0.00635$ (most values $=0$ )

WTsurgery $\mathrm{W}=0.94777, \mathrm{p}$-value $=0.6422$

ANPKOsham $\mathrm{W}=\mathbf{0 . 6 2 3 5 7}$, $\mathrm{p}$-value $=\mathbf{0 . 0 0 0 1 8}$ (one outlier)

ANPKOsurgery $\mathrm{W}=0.91903, \mathrm{p}$-value $=0.3489$

4) Unequal variances: Levene's test $F$ value $=5.6634 p$-value $=0.002943$

Analysis selected: One-way Welch ANOVA (unequal variances):

$F=33.641, p$-value $=4.473 e-07$

\begin{tabular}{|l|c|c|c|}
\hline \multicolumn{4}{|l|}{ Post ANOVA pair-wise Welch t-tests with Bonferroni multi-comparison correction } \\
\hline Groups & p-value (corrected) & Estimates 95\% Cl of diff between means \\
\hline Anpsham-Anpsurgery & $3.2 \mathrm{e}-09$ & -3.547 & -1.798 \\
\hline Wtsham-Anpsham & 1.0000 & -1.105 & 0.6893 \\
\hline Wtsurgery-Anpsham & 0.0071 & 0.2305 & 1.98 \\
\hline Wtsham-Anpsurgery & $5.3 \mathrm{e}-10$ & -3.755 & -2.006 \\
\hline Wtsurgery-Anpsurgery & $\mathbf{6 . 4 e - 0 5}$ & $\mathbf{- 2 . 4 1 9}$ & $-\mathbf{0 . 7 1 6 2}$ \\
\hline Wtsurgery-Wtsham & 0.0011 & 2.1188 & 0.4386 \\
\hline
\end{tabular}

Sensitivity analysis of robustness:

IF normal distribution not assumed:

Kruskal-Wallis test: $\quad$ chi-squared $=31.52, \mathrm{df}=3, \mathrm{p}$-value $=6.606 \mathrm{e}-07$

Post KW pair-wise Mann-Whitney tests with Bonferroni multicomparison correction

\begin{tabular}{|l|c|}
\hline Groups & p-value (corrected) \\
\hline Anpsurgery-Anpsham & 0.0017 \\
\hline Wtsham-Anpsham & 0.0339 \\
\hline Wtsurgery-Anpsham & 0.0031 \\
\hline Wtsham-Anpsurgery & 0.0015 \\
\hline Wtsurgery-Anpsurgery & $\mathbf{0 . 0 2 7 4}$ \\
\hline Wtsurgery-Wtsham & 0.0015 \\
\hline
\end{tabular}

Figure 1J: Quantification of articular cartilage damage of 12 -month old Anp32a ${ }^{-1-}$ mice(ANPKO) $(n=10)$ and WT mice knee joints $(n=10)$ by OARSI severity grade score

Research question: is the severity of articular cartilage damage (OARSI grade severity score) different between WT and ANPKO mice?

Null hypothesis: no difference in OARSI severity grade score between WT and ANPKO mice.

Assumptions: independent groups - normal distribution with similar variances 
1) osteoarthritis model scores in genetically identical mice are commonly normally distributed

2) $\mathrm{QQ}$ (quantile-quantile) plots suggest normal distribution
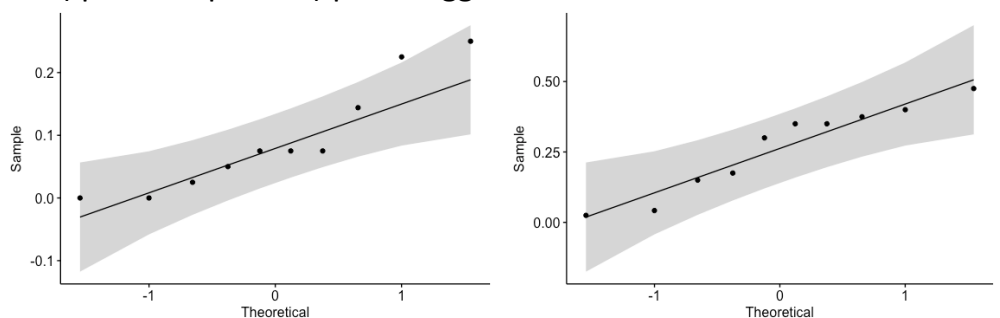

\begin{tabular}{|c|c|c|c|c|c|}
\hline \multicolumn{2}{|c|}{ Analysis selected: Two independent sample t-test (equal variance) } \\
\hline $\mathrm{r}$ & $\begin{array}{c}\text { Shapiro-Wilk test } \\
\text { non-OA }\end{array}$ & $\begin{array}{c}\text { Shapiro-Wilk } \\
\text { test OA }\end{array}$ & Levene test & p-value & $\begin{array}{c}\mathbf{9 5 \%} \mathrm{Cl} \text { of diff. } \\
\text { of } \text { means }\end{array}$ \\
\hline KO vs WT & $\mathrm{W}=0.8721, \mathrm{p}-$ & $\mathrm{W}=0.91614, \mathrm{p}-$ & $\mathrm{F}=2.7967 \quad \mathrm{p}-$ & $\mathrm{p}$-value $=$ & $\mathbf{0 , 0 5 3 4 2}$ to \\
& value $=0.1058$ & value $=0.3259$ & value $=0.1118$ & $\mathbf{0 . 0 0 6 9 6 7}$ & $\mathbf{0 , 2 9 1 2}$ \\
\end{tabular}

Sensitivity analysis of robustness:

IF Normal distribution not assumed:

Mann-Whitney-test: $\quad \mathbf{W}=\mathbf{8 1 . 5}, \mathbf{p}$-value $\mathbf{=} \mathbf{0 . 0 1 8 8}$

\section{Figure 2}

Fig-2A: Volcano plot of the microarray data obtained from tibial articular cartilage of Anp32a-deficient male mice and WT mice ( $n=4$ per group). The volcano plot shows the differentially expressed genes by log 2 ratio (Anp32a $a^{-1}-W T$ mice) as $\mathrm{x}$-axis vs. - $\log 10$ ( $P$-values) as $\mathrm{y}$-axis.

Data were analyzed as published before [2, 3]: differences in gene expression were defined using a modified $t$-test by the limma package from Bioconductor followed by Benjamini-Hochberg multiple testing correction.

Fig-2C: Quantitative PCR data confirming the downregulation of ATM expression in articular chondrocytes from male Anp32a-deficient mice.

No specific statistical analysis was used for this dataset as we hypothesized based on the microarray data that expression of ATM would be virtually absent in the ANP32A knockout mice. The graph shows the difference between groups.

Fig-2H: Expression of ATM measured by quantitative PCR) in articular chondrocytes from hips of osteoarthritic patients (OA) as compared to chondrocytes from non-OA patients $(n=4)$.

Research question: are levels of ATM different between patients with and without osteoarthritis?

Null hypothesis: no difference in expression levels of ATM between OA and non-OA groups.

Assumptions: independent groups - normal distribution with similar variances

1) Gene expression in tissue samples is commonly normally distributed

2) $\mathrm{QQ}$ (quantile-quantile) plots suggest normal distribution

ATM gene expression (non-OA - OA) 

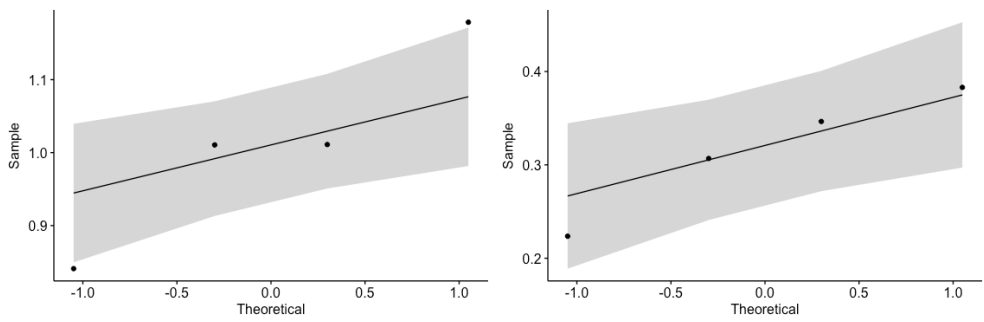

\begin{tabular}{|l|c|c|c|c|c|}
\hline \multicolumn{2}{|c|}{ Analysis selected: Two independent sample t-test (equal variance) } \\
\hline & $\begin{array}{c}\text { Shapiro-Wilk test } \\
\text { non-OA }\end{array}$ & $\begin{array}{c}\text { Shapiro-Wilk } \\
\text { test OA }\end{array}$ & Levene's test & p-value & $\begin{array}{c}\mathbf{9 5 \%} \text { Cl of diff } \\
\text { of means }\end{array}$ \\
\hline OA vs & $\mathrm{W}=0.94543, \mathrm{p}-$ & $\mathrm{W}=0.96101, \mathrm{p}$ - & F-value $=0.439$ & $\mathbf{p}$-value $=$ & $\mathbf{0 . 5 0 7 0 6 9 6}$ to \\
NONOA & value $=0.6877$ & value $=0.7852$ & P-value $=0.5322$ & $\mathbf{0 . 0 0 0 1 0 2 9}$ & $\mathbf{0 . 8 8 3 6 6 5 0}$ \\
\hline
\end{tabular}

\section{Sensitivity analysis of robustness:}

Normal distribution not assumed: Mann-Whitney test $W=16, \mathbf{p}$-value $\mathbf{=} \mathbf{0 . 0 2 8 5 7}$

Fig-2J-K: (J) Expression of ATM determined by RNA sequencing in paired preserved and damaged cartilage isolated from hips (o) and knees $(\Delta)$ from patients with osteoarthritis. The values for the preserved cartilage was set at 0 for each cartilage pair. (K) Correlation between ANP32A and ATM expression levels.

Data were analyzed as published before for the RAAK dataset [1], with differential gene expression being tested pairwise between preserved and lesioned samples followed by Benjamini-Hochberg False Discovery Rate correction. Correlation was tested using the Pearson correlation test.

Fig-2L-M-N: ANP32A and ATM expression measured by quantitative PCR in human articular chondrocytes transfected with siRNA against ANP32A (siANP) or scrambled siRNA (siSCR). Data are from two experiments with three technical replicates

No specific statistical analysis was used for these molecular biology analyses, but the evidence is based on the replication of the effect in an independent sample. Data shown are of three technical replicates with two data points obtained from two independent experiments.

\section{Figure 3}

No specific statistical analysis was used for these molecular biology analyses, but the evidence is based on the replication of the effect in independent samples. Data shown are technical replicates representative of independent experiments as outlined in the figure legend.

\section{Figure 4}

Fig-4B: Quantification of cartilage damage in the knee joints from Anp32a ${ }^{-/-}$mice and WT mice after DMM and NAC treatment, by OARSI severity grade score; $n=2$ (WT-Sham NAC), 5 (WT-Surgery), 5 (WT-Surgery NAC), 2 (Anp32a ${ }^{-/-}$ -Surgery), 5 (Anp32a-- -Surgery NAC).

Research question: Can the increased severity of articular cartilage damage (OARSI grade severity score) ANPKO be reversed by NAC treatment in the DMM model? 
Null hypothesis: no difference in OARSI severity grade score by NAC treatment in ANPKO mice.

Assumptions: independent groups - normal distribution with unequal variances

1) model scores in genetically identical mice are commonly normally distributed

2) $\mathrm{QQ}$ (quantile-quantile) plots suggest normality).

3) Shapiro-Wilk test: WTsurgery $W=0.89956$, $p$-value $=0.4075$

WTsurgeryNAC $W=0.92951, p$-value $=0.593$

ANPKOsurgeryNAC $W=0.9572$, $p$-value $=0.7883$

OARSI score (WTshamNAC-WTsurgery-WTsurgeryNACANPKOsurgery-

ANPKOsurgeryNAC)
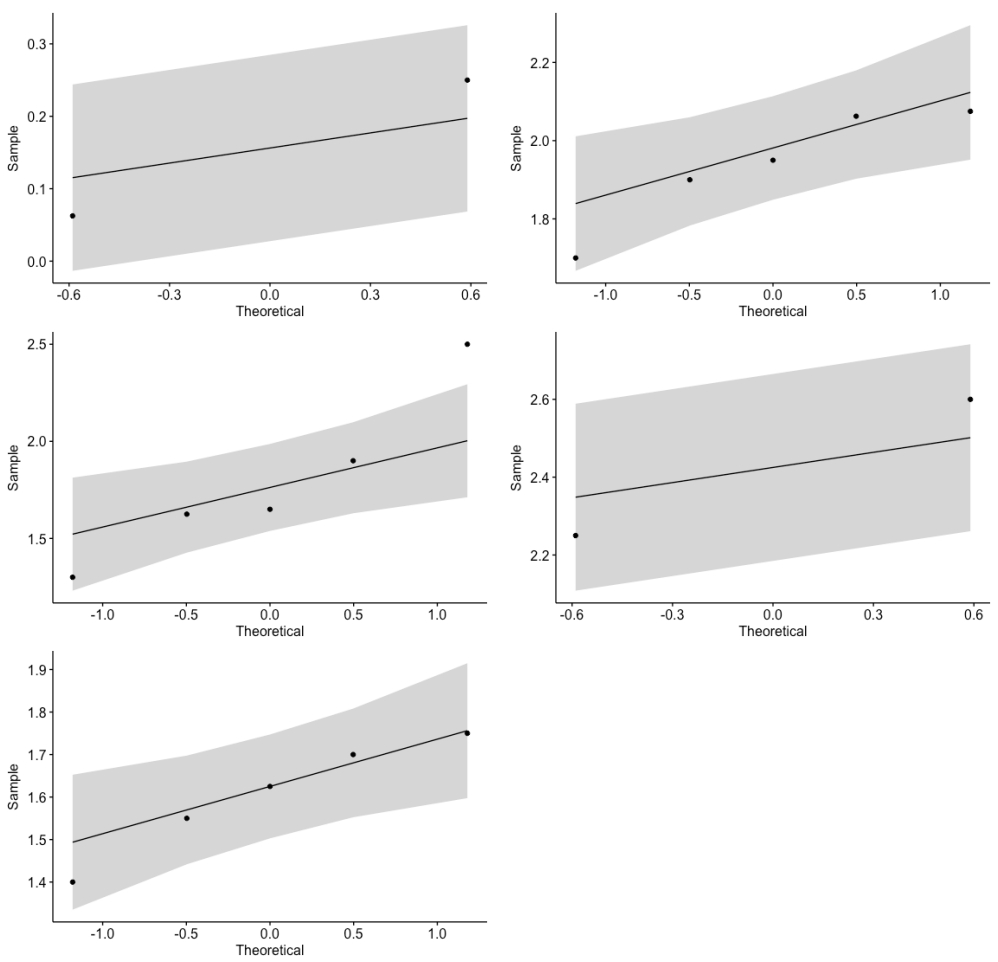

4) Equal variances: Levene's test F value: 0.8628 P-value: 0.5099

\section{Analysis selected: One-way ANOVA:}

$F=20.6, p$-value $=9.51 e-06 e$

\begin{tabular}{|l|c|c|c|}
\hline \multicolumn{5}{|l|}{ Post ANOVA pair-wise t-tests with Bonferroni multi-comparison correction } \\
\hline Groups & $\mathbf{p}$-value (corrected) & Estimates $\mathbf{9 5 \%} \mathbf{C l}$ of diff between means \\
\hline ANPKOsurg-ANPKONACsu & $\mathbf{0 . 0 3 0 2 1}$ & $\mathbf{0 . 0 5 8 0 5}$ & $\mathbf{1 . 5 8 2}$ \\
\hline WTshamNAC-ANPKONACsu & 0.00019 & -2.211 & -0.6868 \\
\hline WTsurgery-ANPKONACsu & 0.75489 & -0.2435 & 0.9085 \\
\hline WTsurgeryNAC-ANPKONACsu & 1.00000 & -0.386 & 0.766 \\
\hline WTshamNAC-ANPKOsurgery & $9.1 \mathrm{e}-06$ & -3.179 & -1.358 \\
\hline WTsurgery-ANPKOsurgery & 0.51604 & -1.249 & 0.2745 \\
\hline WTsurgNAC-ANPKOsurgery & 0.15652 & -1.392 & 0.132 \\
\hline WTsurgery-WTshamNAC & $1.9 \mathrm{e}-05$ & 1.019 & 2.543 \\
\hline WTsurgeryNAC-WTshamNAC & $4.9 \mathrm{e}-05$ & 0.8768 & 2.401 \\
\hline
\end{tabular}




\begin{tabular}{|l|l|l|l|}
\hline WTsurgeryNAC-WTsurgery & 1.000 & -0.7185 & 0.4335 \\
\hline
\end{tabular}

\section{Sensitivity analysis of robustness:}

IF normal distribution not assumed:

Kruskal-Wallis test: $\quad$ chi-squared $=11.725, \mathrm{df}=4, \mathrm{p}$-value $\mathbf{= 0 . 0 1 9 5 2}$

Post-hoc tests have no power given low $n$

\section{Comparison including OARSI scores Fig 1 and Fig 4}

Analysis selected: One-way ANOVA:

F-value: 10.58 - P-value $=0.000355$

Post KW pair-wise Mann-Whitney tests with Bonferroni multicomparison correction

\begin{tabular}{|l|c|}
\hline Groups & p-value (corrected) \\
\hline Anpsurgery-AnpsurgeryNAC & $\mathbf{0 . 0 1 3 7 4}$ \\
\hline Wtsurgery-Anpsurgery & 0.00043 \\
\hline Wtsurgery-ANPsurgeryNAC & 1 \\
\hline
\end{tabular}

Fig-4E: Quantification of type X collagen staining in the articular cartilage from Anp32a $a^{-/-}$mice compared to WT mice, with or without NAC treatment, by digital image analysis ( $\mathrm{n}=3$ per group)

Research question: Are type X collagen levels increased in articular cartilage of ANPKO mice compared to wildtype mice and what is the effect of NAC treatment hereon?

Null hypothesis: 1 . no difference in type $X$ collagen staining between ANPKO and WT mice

2. no effect of NAC on type $X$ collagen staining in ANPKO mice

Assumptions: independent groups - normal distribution with unequal variances

1) Protein levels in genetically identical mice are commonly normally distributed

2) $\mathrm{QQ}$ (quantile-quantile) plots suggest normality).

3) Shapiro-Wilk test: WT: reference values

ANPKO: $W=0.92872$, $\mathrm{p}$-value $=0.4838$

WTNAC: $\mathrm{W}=0.81401, \mathrm{p}$-value $=0.1484$

ANPKONAC $W=0.95479, p$-value $=0.5908$

ColX levels (WT (ref 100\%) -ANPKO -WTNAC - ANPKO NAC)
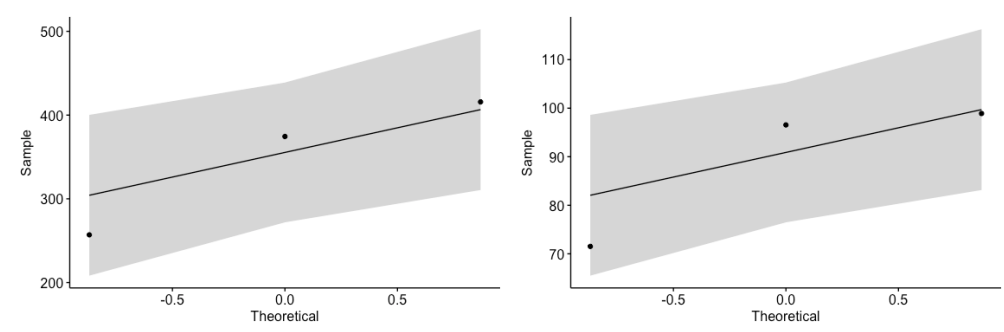


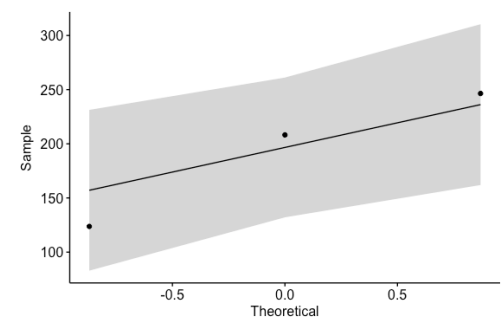

4) Equal variances: Levene's test F-value $=1.379-$ P-value $=0.3175$

\section{Analysis selected: One-way ANOVA:}

$F=15.85$ p-value $=0.000997$

\begin{tabular}{|l|c|c|c|}
\hline \multicolumn{4}{|l|}{ Post ANOVA pair-wise t-tests with Bonferroni multi-comparison correction } \\
\hline Groups & p-value (corrected) & \multicolumn{2}{|c|}{ Estimates 95\% Cl of diff between means } \\
\hline ANPKO-ANPKONAC & $\mathbf{0 . 0 3 8 7}$ & 7.613 & 304.9 \\
\hline WT-ANPKO & $\mathbf{0 . 0 0 2 4}$ & -397.7 & -100.4 \\
\hline WTNAC-ANPKO & 0.0018 & -408.7 & -111.4 \\
\hline WT-ANPKONAC & 0.3701 & -241.4 & 55.86 \\
\hline WTNAC-ANPKONAC & 0.2475 & -252.5 & 44.85 \\
\hline WT-WTNAC & 1 & -137.6 & 159.7 \\
\hline
\end{tabular}

Sensitivity analysis of robustness:

Kruskal-Wallis test: $\quad$ chi-squared $=10.532, \mathrm{df}=3, \mathbf{p}$-value $\mathbf{= 0 . 0 1 4 5 5}$

Post-hoc tests have no power given low $n$

\section{Figure 5}

Fig-5A: ATM expression measured by quantitative PCR, in adult brain from 8-week-old WT and Anp32a-deficient mice $(n=9)$

No specific statistical analysis was used for this dataset as we hypothesized based on the microarray data that expression of ATM would be virtually absent in the ANP32A knockout mice. The graph shows the difference between groups.

Fig-5F: The average stride length was shorter for Anp32a-deficient mice than for WT mice in both front-paw (FP) and hind-paw (HP), a defect that was rescued by NAC treatment.

Research question: Are gait parameters (stride length) different between WT and ANPKO mice and what is the effect of NAC on such differences?

Null hypothesis: 1 . no difference in stride length between ANPKO and WT mice

2. no effect of NAC on stride length in ANPKO mice

Assumptions: independent groups - normal distribution with equal variances

\section{FRONT PAWS}

1) parameters in genetically identical mice are commonly normally distributed

2) $\mathrm{QQ}$ (quantile-quantile) plots suggest normality).

3) Shapiro-Wilk test: WT $W=0.90819, p$-value $=0.4568$

ANPKO $\mathrm{W}=0.91588, \mathrm{p}$-value $=0.4381$ 


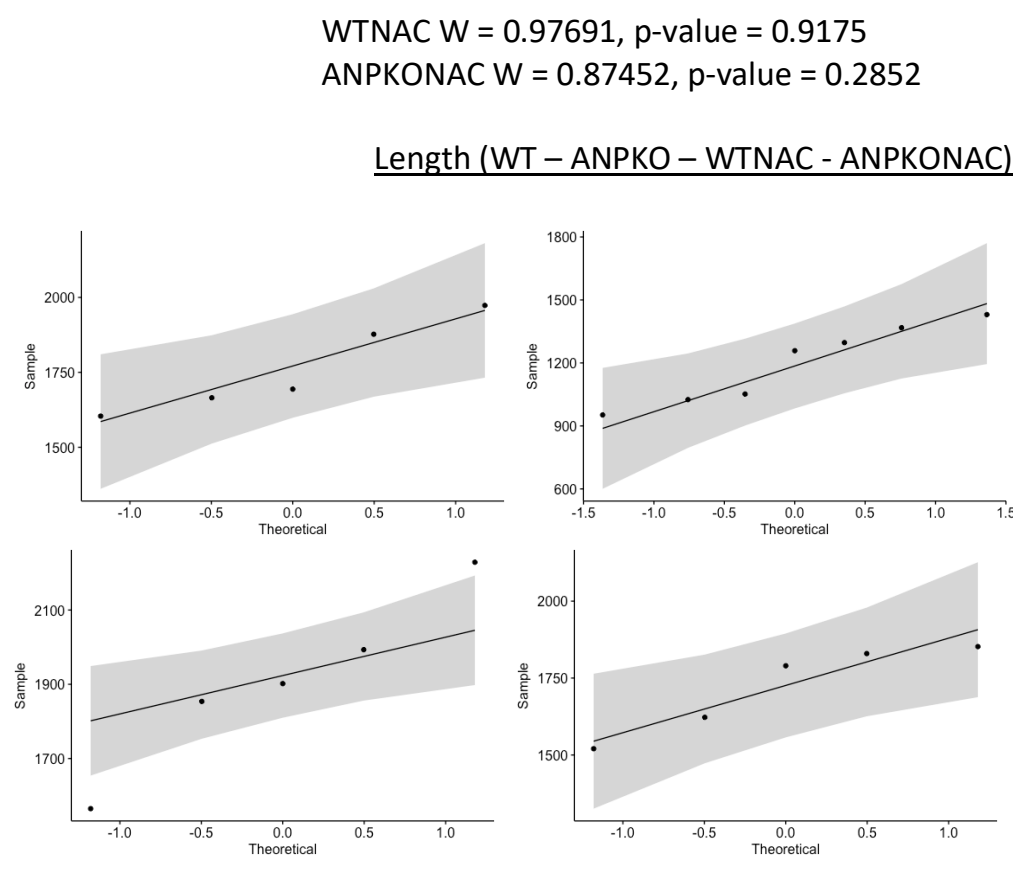

4) Equal variances: Levene's test $F$ value $=0.2359$-value $=0.8701$

Analysis selected: One-way ANOVA:

$F=17.61, p$-value $=1.37 e-05 e$

\begin{tabular}{|l|c|c|c|}
\hline \multicolumn{4}{|l|}{ Post ANOVA pair-wise t-tests with Bonferroni multi-comparison correction } \\
\hline Groups & p-value (corrected) & \multicolumn{2}{|c|}{ Estimates 95\% Cl of diff between means } \\
\hline ANPKONAC-ANPKO & $\mathbf{0 . 0 0 0 7 9}$ & $\mathbf{3 0 2 . 7}$ & $\mathbf{7 4 8 . 1}$ \\
\hline WT-ANPKO & $\mathbf{0 . 0 0 0 3 6}$ & $\mathbf{3 3 7 . 5}$ & $\mathbf{7 9 2 . 8}$ \\
\hline WTNAC-ANPKO & $2.2 \mathrm{e}-05$ & 437.8 & 984.1 \\
\hline WT-ANPKONAC & 1.00000 & -179.3 & 258.9 \\
\hline WTNAC-ANPKONAC & 0.78624 & -103.6 & 474.7 \\
\hline WTNAC-WT & 1.00000 & -149.4 & 440.9 \\
\hline
\end{tabular}

\section{Sensitivity analysis of robustness:}

IF normal distribution not assumed:

Kruskal-Wallis test: $\quad$ chi-squared $=15.09, \mathrm{df}=3, \mathrm{p}$-value $=0.001741$

Post KW pair-wise Mann-Whitney tests with Bonferroni multicomparison correction

\begin{tabular}{|l|c|}
\hline Groups & p-value (corrected) \\
\hline ANPKONAC-ANPKO & $\mathbf{0 . 0 1 5}$ \\
\hline WT-ANPKO & $\mathbf{0 . 0 1 5}$ \\
\hline WTNAC-ANPKO & 0.015 \\
\hline WT-ANPKONAC & 1.00000 \\
\hline WTNAC-ANPKONAC & 0.571 \\
\hline WTNAC-WT & 1 \\
\hline
\end{tabular}




\section{HIND PAWS}

1) parameters in genetically identical mice are commonly normally distributed

2) $Q Q$ (quantile-quantile) plots suggest normality).

3) Shapiro-Wilk test: $\quad$ WT $W=0.86962, p$-value $=0.2649$ ANPKO $W=0.96348, p$-value $=0.848$ WTNAC $W=0.87097, p$-value $=0.2704$ ANPKONAC $W=0.98349, p$-value $=0.9524$

\section{Length (WT - ANPKO - WTNAC - ANPKONAC)}
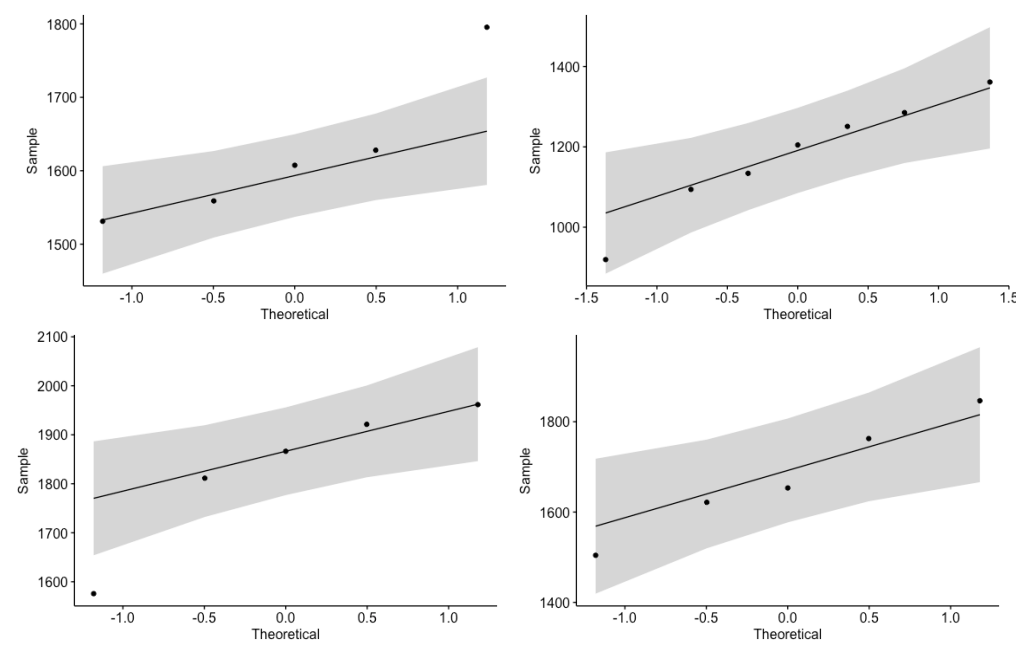

4) Equal variances: Levene test $F$ value $=: 0.2053$ P-value $=0.8914$

Analysis selected: One-way ANOVA:

$F=26.41, p$-value $=8.22 e-07$

\begin{tabular}{|l|c|c|c|}
\hline \multicolumn{5}{|l|}{ Post ANOVA pair-wise t-tests with Bonferroni multi-comparison correction } \\
\hline Groups & $\mathbf{p}$-value (corrected) & \multicolumn{2}{|c|}{ Estimates 95\% Cl of diff between means } \\
\hline ANPKONAC-ANPKO & $\mathbf{3 . 8 e - 0 5}$ & $\mathbf{3 1 6 . 1 7}$ & $\mathbf{6 8 2 . 1}$ \\
\hline WT-ANPKO & $\mathbf{0 . 0 0 0 1 5}$ & $\mathbf{2 7 5 . 7 0}$ & $\mathbf{6 1 5 . 5}$ \\
\hline WTNAC-ANPKO & $1.1 \mathrm{e}-06$ & 455.65 & 841.8 \\
\hline WT-ANPKONAC & 1.00000 & -226.13 & 119.1 \\
\hline WTNAC-ANPKONAC & 0.58913 & -57.59 & 356.8 \\
\hline WTNAC-WT & 0.17578 & 14.02 & 392.2 \\
\hline
\end{tabular}

Sensitivity analysis of robustness:

IF normal distribution not assumed:

Kruskal-Wallis test: $\quad$ chi-squared $=15.944, d f=3, p$-value $=0.001164$

Post KW pair-wise Mann-Whitney tests with Bonferroni multicomparison correction

\begin{tabular}{|l|c|}
\hline Groups & p-value (corrected) \\
\hline ANPKONAC-ANPKO & 0.015 \\
\hline WT-ANPKO & 0.015 \\
\hline
\end{tabular}




\begin{tabular}{|l|c|}
\hline WTNAC-ANPKO & 0.015 \\
\hline WT-ANPKONAC & 1.00000 \\
\hline WTNAC-ANPKONAC & 0.905 \\
\hline WTNAC-WT & 0.333 \\
\hline
\end{tabular}

Fig-5G: 8-week old WT mice had more consistent walking patterns, as revealed by a higher regularity index compared to 8-week old Anp32a-deficient mice. This defect was rescued by NAC treatment.

Research question: Are gait parameters (regularity index) different between WT and ANPKO mice and what is the effect of NAC on such differences?

Null hypothesis: 1 . no difference in regularity index between ANPKO and WT mice

2. no effect of NAC on regularity index in ANPKO mice

Assumptions: independent groups - normal distribution with equal variances

1) parameters in genetically identical mice are commonly normally distributed

2) $\mathrm{QQ}$ (quantile-quantile) plots suggest normality).

3) Shapiro-Wilk test: $\quad W T W=0.7573, p$-value $=0.03473$

ANPKO $\mathrm{W}=0.95154, \mathrm{p}$-value $=0.7437$

WT NAC W $=0.55218, \mathrm{p}$-value $=0.000131$

ANPKONAC $W=0.88104, p$-value $=0.314$
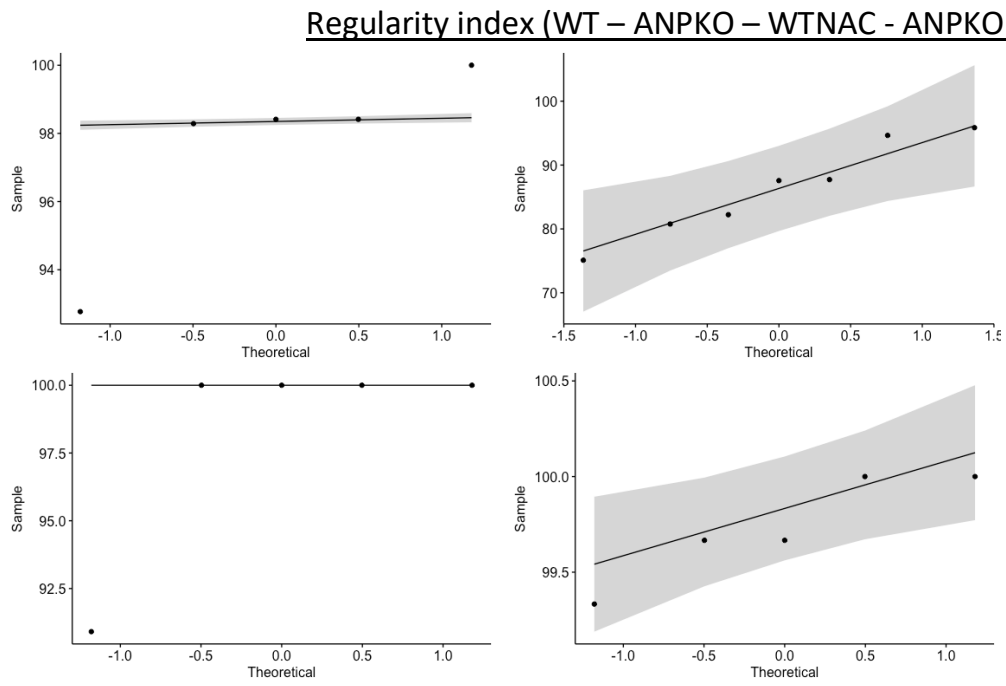

4) Equal variances: Levene's test F-value $=3.0724, p$-value $=0.05413$

Analysis selected: One-way ANOVA with unequal variances:

$F=7.4162$, $p$-value $=0.01194$

\begin{tabular}{|l|c|c|c|}
\hline \multicolumn{5}{|l|}{ Post ANOVA pair-wise t-tests with Bonferroni multi-comparison correction } \\
\hline Groups & $\mathbf{p}$-value (corrected) & \multicolumn{2}{|c|}{ Estimates 95\% Cl of diff between means } \\
\hline ANPKONAC-ANPKO & $\mathbf{0 . 0 0 1 1}$ & $\mathbf{6 . 5 3 4}$ & $\mathbf{2 0 . 3 9 7}$ \\
\hline WT-ANPKO & $\mathbf{0 . 0 0 5 9}$ & $\mathbf{4 . 1 8 9}$ & $\mathbf{1 8 . 4 3 0}$ \\
\hline WTNAC-ANPKO & 0.0037 & 4.365 & 19.464 \\
\hline WT-ANPKONAC & 1.00000 & -5.597 & 1.284 \\
\hline WTNAC-ANPKONAC & 1.00000 & -6.592 & 3.490 \\
\hline WTNAC-WT & 1.00000 & -4.592 & 5.802 \\
\hline
\end{tabular}

\section{Sensitivity analysis of robustness:}


IF normal distribution not assumed:

Kruskal-Wallis test: $\quad$ chi-squared $=13.676, \mathrm{df}=3, \mathrm{p}$-value $=0.003381$

Post KW pair-wise Mann-Whitney tests with Bonferroni multicomparison correction

\begin{tabular}{|l|c|}
\hline Groups & p-value (corrected) \\
\hline ANPKONAC-ANPKO & $\mathbf{0 . 0 3 4}$ \\
\hline WT-ANPKO & 0.088 \\
\hline WTNAC-ANPKO & 0.079 \\
\hline WT-ANPKONAC & 0.532 \\
\hline WTNAC-ANPKONAC & 1 \\
\hline WTNAC-WT & 1 \\
\hline
\end{tabular}

\section{Figure 6}

Fig-6A: Subcapital dual energy X-ray absorptiometry (DEXA) analysis of bone mineral density (BMD), bone mineral content (BMC) and lean body mass in 12-week old female Anp32a ${ }^{-/}$mice $(n=9)$ compared to WT littermates $(n=11)$

Research question: Are bone parameters different between WT and ANPKO mice?

Null hypothesis: 1 . no difference in bone parameters between ANPKO and WT mice

Assumptions: independent groups - normal distribution with similar variances

1) Bone density parameters in genetically identical mice are commonly normally distributed

2) $\mathrm{QQ}$ (quantile-quantile) plots suggest normal distribution for the different parameters
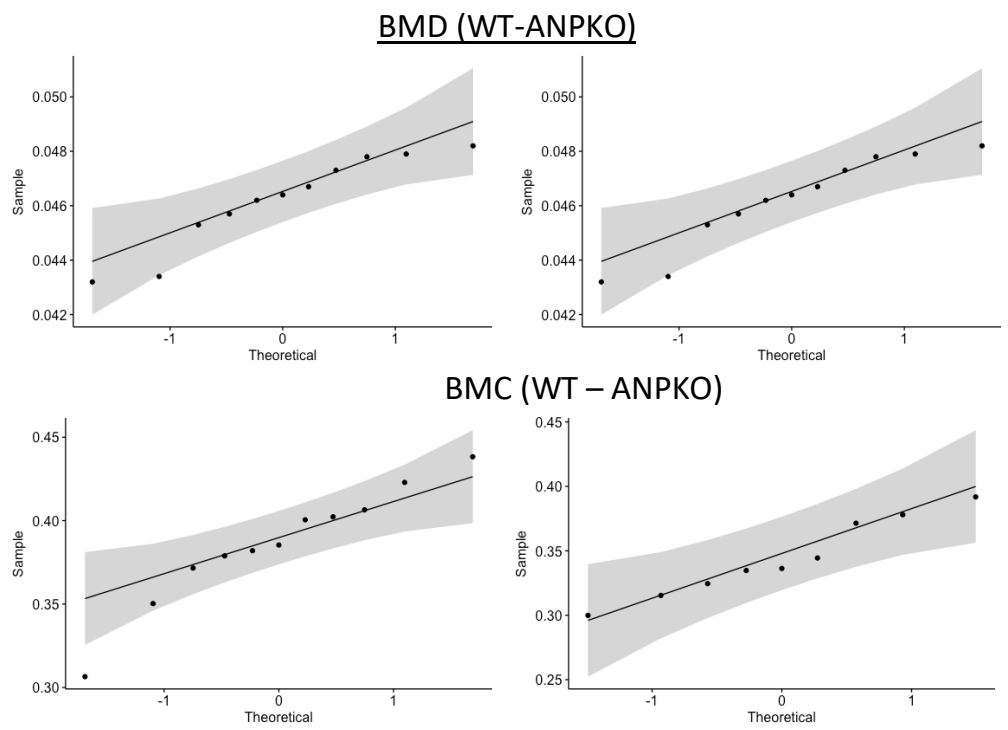

LEAN MASS (WT - ANPKO) 

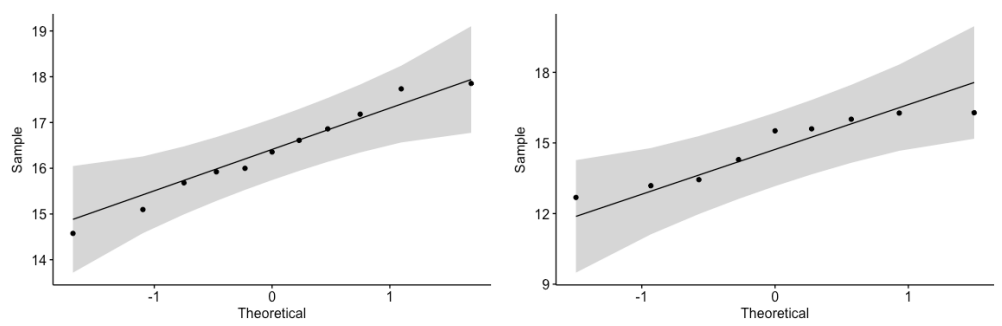

\begin{tabular}{|c|c|c|c|c|c|}
\hline \multicolumn{6}{|c|}{ Analysis selected: Two independent sample t-test } \\
\hline $\begin{array}{c}\text { ANPKO vs } \\
\text { WT }\end{array}$ & $\begin{array}{c}\text { Shapiro-Wilk test } \\
\text { WT }\end{array}$ & $\begin{array}{c}\text { Shapiro-Wilk test } \\
\text { ANPKO }\end{array}$ & Levene test & p-value & $\begin{array}{c}95 \% \mathrm{Cl} \text { of diff } \\
\text { of means }\end{array}$ \\
\hline BMD & $\begin{array}{l}W=0.90904, p- \\
\text { value }=0.2376\end{array}$ & $\begin{array}{l}W=0.96261, p- \\
\text { value }=0.8252\end{array}$ & $\begin{array}{l}\text { F-value }=0.0273 \\
p \text {-value }=0.8705\end{array}$ & $\begin{array}{c}p \text {-value }= \\
0.03916\end{array}$ & $\begin{array}{c}-0.00328 \text { to }- \\
0.000093\end{array}$ \\
\hline BMC & $\begin{array}{l}W=0.94778, p- \\
\text { value }=0.6157\end{array}$ & $\begin{array}{l}W=0.9571, p- \\
\text { value }=0.7676\end{array}$ & $\begin{array}{l}\text { F-value }=0.0453 \\
p \text {-value }=0.8339\end{array}$ & $\begin{array}{c}p \text {-value }= \\
0.01257\end{array}$ & $\begin{array}{c}-0.07363 \text { to }- \\
0.01013802 \\
\end{array}$ \\
\hline Lean mass & $\begin{array}{l}W=0.97555, p- \\
\text { value }=0.9364\end{array}$ & $\begin{array}{l}W=0.8712, p- \\
\text { value }=0.1267\end{array}$ & $\begin{array}{l}\text { F-value }=0.9833 \\
p \text {-value }=0.3345\end{array}$ & $\begin{array}{c}p \text {-value }= \\
0.01153\end{array}$ & $\begin{array}{c}-2.6959558 \text { to }- \\
0.3903508\end{array}$ \\
\hline
\end{tabular}

Sensitivity analysis of robustness:

Normal distribution not assumed: Mann-Whitney-test:

BMD: $W=24, p$-value $=0.05619$

BMC: $W=16, p$-value $\mathbf{=} \mathbf{0 . 0 0 9 6 9 3}$

Lean mass: $W=19, \mathbf{p}$-value $\mathbf{=} \mathbf{0 . 0 2 0 0 4}$

Fig-6B: Peripheral quantitative computed tomography analysis (pQCT) of trabecular and cortical bone parameters in femora from 12-week old female Anp32 $a^{-/-}$mice $(n=9)$ and WT littermates $(n=10)$

Research question: Are bone parameters different between WT and ANPKO mice? Null hypothesis: 1 . no difference in bone parameters between ANPKO and WT mice Assumptions: independent groups - normal distribution with similar variances

1) Bone density parameters in genetically identical mice are commonly normally distributed

2) $\mathrm{QQ}$ (quantile-quantile) plots suggest normal distribution for the different parameters

\section{$\underline{\text { Trabecular bone content (WT-ANPKO) }}$}

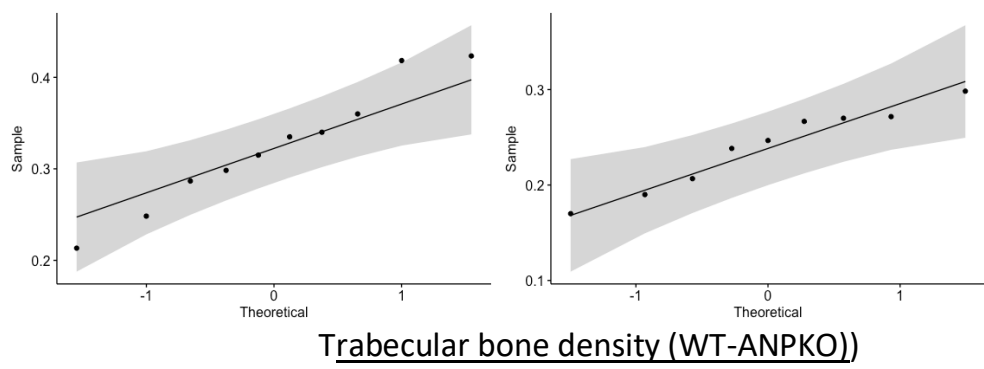



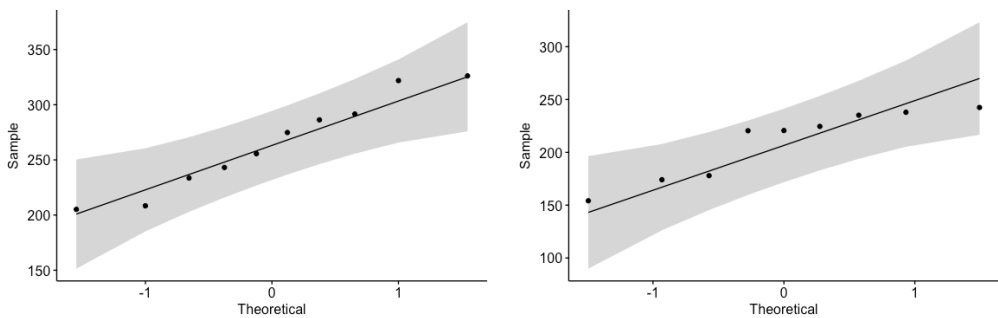

Trabecular bone area (WT - ANPKO)
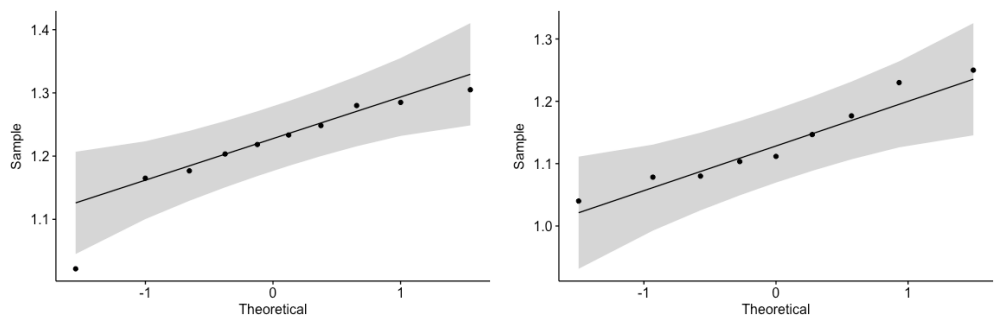

Cortical bone content (WT-ANPKO)
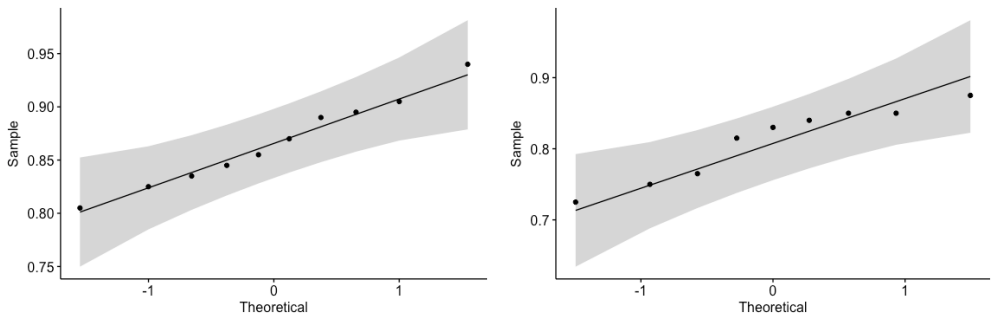

Cortical bone density (WT-ANPKO)
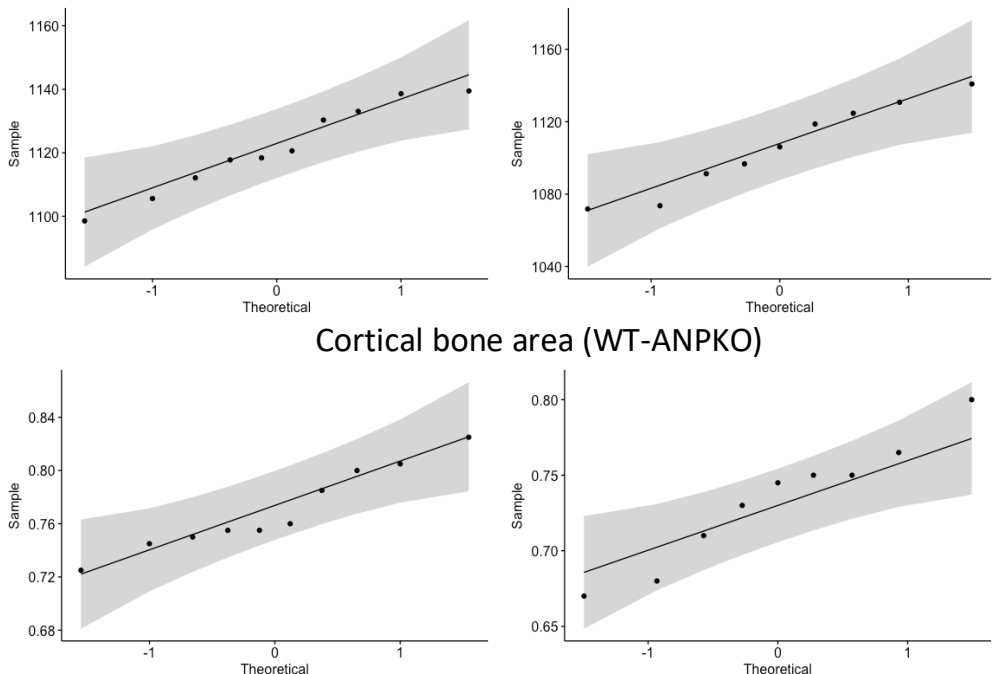

\begin{tabular}{|c|c|c|c|c|c|}
\hline \multicolumn{6}{|c|}{ Analysis selected: Two independent sample t-test } \\
\hline $\begin{array}{c}\text { ANPKO vs } \\
\text { WT }\end{array}$ & $\begin{array}{c}\text { Shapiro-Wilk test } \\
\text { WT }\end{array}$ & $\begin{array}{c}\text { Shapiro-Wilk test } \\
\text { ANPKO }\end{array}$ & Levene test & p-value & $\begin{array}{c}\mathbf{9 5 \%} \mathrm{Cl} \text { of diff } \\
\text { of } \text { means }\end{array}$ \\
\hline $\begin{array}{c}\text { Trabecular } \\
\text { bone cont }\end{array}$ & W $=0.96734, \mathrm{p}-$ & $\mathrm{W}=0.94363, \mathrm{p}-$ & F-value $=1.376$ & $\mathbf{p}$-value $=$ & $\mathbf{- 0 . 1 3 9 2 1 6 8 7}$ to \\
value $=0.8652$ & value $=0.6205$ & p-value $=0.257$ & $\mathbf{0 . 0 0 5 1 2 1}$ & $\mathbf{- 0 . 0 2 8 8 1 9 7 5}$ \\
\hline
\end{tabular}




\begin{tabular}{|c|c|c|c|c|c|}
\hline $\begin{array}{l}\text { Trabecular } \\
\text { bone dens }\end{array}$ & $\begin{array}{l}W=0.94677, p- \\
\text { value }=0.6305\end{array}$ & $\begin{array}{l}W=0.85485, p- \\
\text { value }=0.08424\end{array}$ & $\begin{array}{l}\text { F-value }=1.3347 \\
p \text {-value }=0.2639\end{array}$ & $\begin{array}{l}p \text {-value }= \\
0.006144\end{array}$ & $\begin{array}{c}-92.07739 \text { to }- \\
17.87887\end{array}$ \\
\hline $\begin{array}{l}\text { Trabecular } \\
\text { bone area }\end{array}$ & $\begin{array}{l}W=0.88182, p- \\
\text { value }=0.1369\end{array}$ & $\begin{array}{l}\mathrm{W}=0.93934, \mathrm{p}- \\
\text { value }=0.5749\end{array}$ & $\begin{array}{c}\text { F-value }=0.0015 \\
p \text {-value }=0.97\end{array}$ & $\begin{array}{c}p \text {-value }= \\
0.04069\end{array}$ & $\begin{array}{c}-0.15323050 \text { to } \\
-0.00373225 \\
\end{array}$ \\
\hline $\begin{array}{l}\text { Cortical } \\
\text { bone cont }\end{array}$ & $\begin{array}{l}W=0.98155, p- \\
\text { value }=0.9729\end{array}$ & $\begin{array}{l}W=0.90722, p- \\
\text { value }=0.2969\end{array}$ & $\begin{array}{l}\text { F-value }=0.2316 \\
p \text {-value }=0.6365\end{array}$ & $\begin{array}{c}p \text {-value }= \\
0.01923\end{array}$ & $\begin{array}{c}-0.10057783 \text { to } \\
-0.01019995 \\
\end{array}$ \\
\hline $\begin{array}{l}\text { Cortical } \\
\text { bone dens }\end{array}$ & $\begin{array}{l}W=0.95208, p- \\
\text { value }=0.6931\end{array}$ & $\begin{array}{l}W=0.94828, p- \\
\text { value }=0.6712\end{array}$ & $\begin{array}{l}F \text {-value }=3.9163 \\
p \text {-value }=0.0642\end{array}$ & $\begin{array}{c}p \text {-value }= \\
0.1071\end{array}$ & $\begin{array}{c}-34.449199 \text { to } \\
3.691422\end{array}$ \\
\hline $\begin{array}{l}\text { Cortical } \\
\text { bone area }\end{array}$ & $\begin{array}{l}W=0.93742, p- \\
\text { value }=0.5246\end{array}$ & $\begin{array}{l}W=0.95776, p- \\
\text { value }=0.7746\end{array}$ & $\begin{array}{l}\text { F-value }=0.2677 \\
p \text {-value }=0.6116\end{array}$ & $\begin{array}{c}p \text {-value }= \\
0.04012\end{array}$ & $\begin{array}{r}-0.072451159 \\
\text { to }-0.00188217\end{array}$ \\
\hline
\end{tabular}

Sensitivity analysis of robustness:

Normal distribution not assumed: Mann-Witney-test:

Trabecular bone content: $W=11.5, p$-value $=\mathbf{0 . 0 0 7 0 2 6}$

Trabecular bone density: $\mathrm{W}=15, \mathbf{p}$-value $\mathbf{= 0 . 0 1 3 2 7}$

Trabecular bone area: $\mathrm{W}=20.5, \mathrm{p}$-value $\mathbf{= 0 . 0 4 9 9 4}$

Cortical bone content: $\mathrm{W}=20, \mathrm{p}$-value $\mathbf{=} \mathbf{0 . 0 4 5 3 6}$

Cortical bone density: $W=30, p$-value $=0.2428$

Cortical bone area: $\mathrm{W}=21, \mathrm{p}$-value $=0.05427$

Fig-6E: Histomorphometry analysis of tibiae from male Anp32a\% mice treated $(n=5)$ or not with NAC $(n=5)$ for 13 weeks from the age of 3 weeks until the age of 16 weeks

Research question: Can abnormal bone parameters in ANPKO mice be reversed by NAC treatment?

Null hypothesis: No difference bone parameters by NAC treatment in ANPKO mice.

Assumptions: independent groups - normal distribution with similar variances

1) Bone density parameters in genetically identical mice are commonly normally distributed

2) $\mathrm{QQ}$ (quantile-quantile) plots suggest normal distribution for the different parameters

Bone Area (WT-ANPKO)
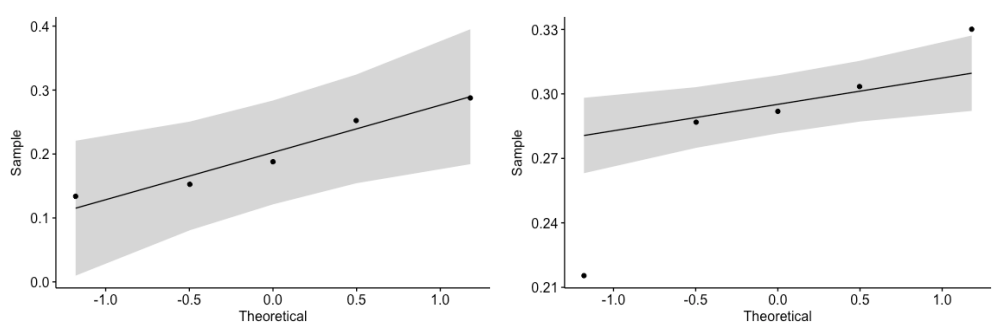

Bone Volume/Total Volume (WT-ANPKO))
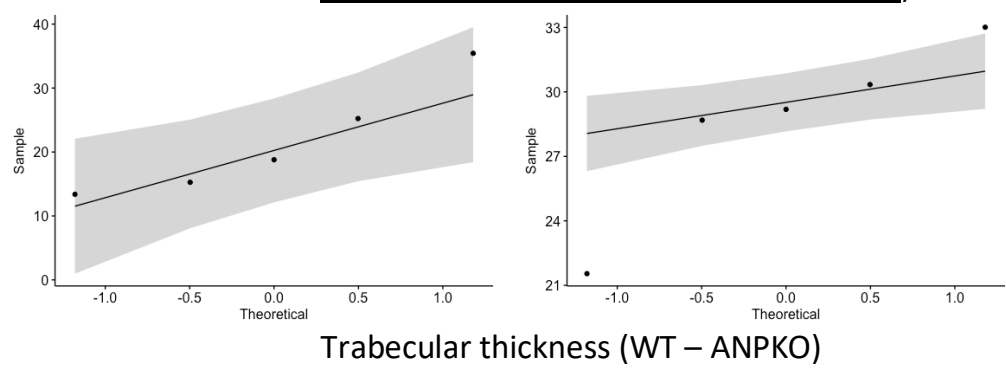

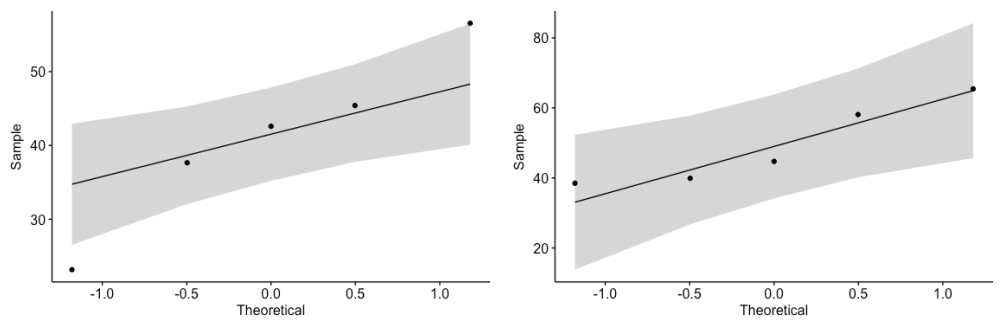

Cortical thickness (WT-ANPKO)
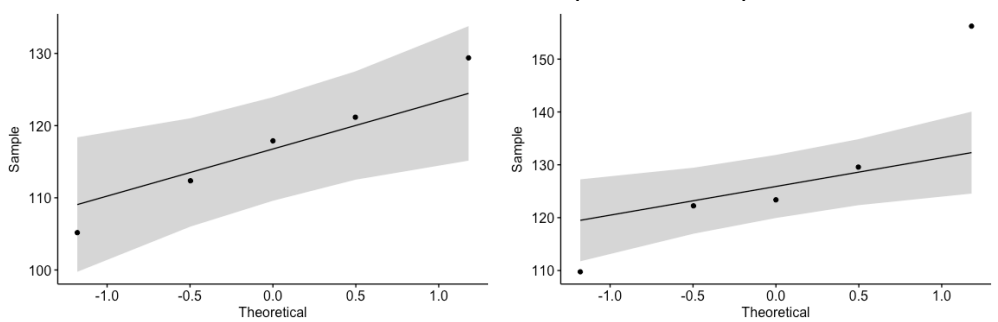

\begin{tabular}{|l|c|c|c|c|c|}
\hline \multicolumn{7}{|l|}{ Analysis selected: Two independent sample t-test } \\
\hline $\begin{array}{l}\text { ANPKO vs } \\
\text { WT }\end{array}$ & $\begin{array}{c}\text { Shapiro-Wilk test } \\
\text { ANPKO }\end{array}$ & $\begin{array}{c}\text { Shapiro-Wilk test } \\
\text { ANPKONAC }\end{array}$ & Levene test & p-value & $\begin{array}{c}\mathbf{9 5 \%} \text { Cl of diff } \\
\text { of } \text { means }\end{array}$ \\
\hline Bone area & $\mathrm{W}=0.93022, \mathrm{p}-$ & $\mathrm{W}=0.88592, \mathrm{p}-$ & F-value $=1.2699$ & $\mathbf{p}$-value $=$ & $\mathbf{- 0 . 1 6 3 2 2 7 9 0}$ to \\
& value $=0.5979$ & value $=0.337$ & $\mathrm{p}$-value $=0.292$ & $\mathbf{0 . 0 4 5 5 2}$ & $\mathbf{- 0 . 0 0 2 1 0 2 4 9 6}$ \\
\hline BV/TV & $\mathrm{W}=0.90933, \mathrm{p}-$ & $\mathrm{W}=0.88592, \mathrm{p}-$ & $\mathrm{F}$-value $=1.464$ & $\mathrm{p}$-value $=$ & -17.151857 to \\
& value $=0.4636$ & value $=0.337$ & $\mathrm{p}$-value $=0.2608$ & 0.1563 & 3.285457 \\
\hline Trabecular & $\mathrm{W}=0.97627, \mathrm{p}-$ & $\mathrm{W}=0.88159, \mathrm{p}$ - & F-value $=0.0244$ & $\mathrm{p}$-value $=$ & -25.793874 to \\
thickness & value $=0.9138$ & value $=0.3166$ & $\mathrm{p}$-value $=0.8798$ & 0.3091 & 9.276874 \\
\hline Cortical & $\mathrm{W}=0.99739, \mathrm{p}-$ & $\mathrm{W}=0.89879, \mathrm{p}$ - & F-value $=0.497$ & $\mathrm{p}$-value $=$ & -31.135752 to \\
thickness & value $=0.9981$ & value $=0.4032$ & $\mathrm{p}$-value $=0.54$ & 0.2409 & 9.05735 \\
\hline
\end{tabular}

Sensitivity analysis of robustness:

Normal distribution not assumed: Mann-Witney-tests:

Bone area: $\mathrm{W}=3, \mathrm{p}$-value $=0.05556$

BV/TV: $\mathrm{W}=6, \mathrm{p}$-value $=0.2222$

Trabecular thickness: $\mathrm{W}=8, \mathrm{p}$-value $=0.4206$

Cortical thickness: $W=6, p$-value $=0.2222$ 


\section{Supplementary Figure 1}

Suppl-Fig-1B: Comparison of knee joints from WT and Anp32a/- mice injected with collagenase, by OARSI severity grade score

Research question: is the severity of articular cartilage damage (OARSI grade severity score) different between WT and ANPKO mice?

Null hypothesis: no difference in OARSI severity grade score between WT and ANPKO mice.

Assumptions: independent groups - normal distribution with unequal variances

1) model scores in genetically identical mice are commonly normally distributed

2) QQ (quantile-quantile) plots suggest normality.

3) Shapiro-Wilk test: WTPBS $W=0.92097, p$-value $=0.1993$

WTcol $W=0.90628, p$-value $=0.1391$

ANPKOPBS $W=0.78195, p$-value $=0.003011$

ANPKOcol $\mathrm{W}=0.94434, \mathrm{p}$-value $=0.4401$

OARSI score (WTPBS-WTcol-ANPKOPBS-ANPKO col)
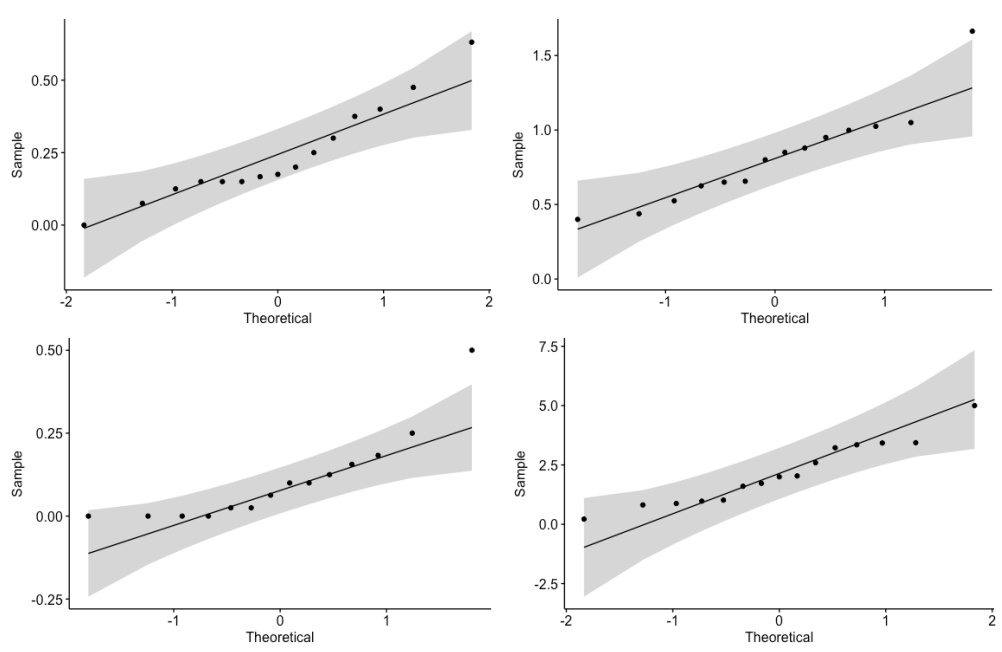

4) Unequal variances: Levene's test $F$ value $=18.445 \mathrm{p}$-value $=2.3 e-08$

\section{Analysis selected: One-way Welch ANOVA (unequal variances):}

$F=28.433, p$-value $=1.227 e-08$

\begin{tabular}{|l|c|c|c|}
\hline \multicolumn{4}{|l|}{ Post ANOVA pair-wise Welch t-tests with Bonferroni multi-comparison correction } \\
\hline Groups & p-value (corrected) & \multicolumn{2}{|c|}{ Estimates 95\% Cl of diff between means } \\
\hline Anpcol-Anppbs & $1.0 \mathrm{e}-09$ & 1.331 & 2.757 \\
\hline Wtpbs-Anppbs & 1.0000 & -0.5808 & 0.8457 \\
\hline Wtscol-Anppbs & 0.057 & -0.01241 & 1.438 \\
\hline Wtpbs-Anpcol & $4.3 \mathrm{e}-09$ & -2.612 & -1.211 \\
\hline Wtcol-Anpcol & $\mathbf{2 . 6 e - 0 5}$ & $\mathbf{- 2 . 0 4 4}$ & $-\mathbf{0 . 6 1 7 8}$ \\
\hline Wtpbs-Wtcol & 0.180 & -1.294 & 0.1327 \\
\hline
\end{tabular}

Sensitivity analysis of robustness:

IF normal distribution not assumed: 
Kruskal-Wallis test: $\quad$ chi-squared $=43.662, \mathrm{df}=3, \mathrm{p}$-value $=1.78 \mathrm{e}-09$

Post KW pair-wise Mann-Whitney tests with Bonferroni multicomparison correction

\begin{tabular}{|l|c|}
\hline Groups & p-value (corrected) \\
\hline Anpscol-Anppbs & $4.5 \mathrm{e}-05$ \\
\hline Wtscol-Anppbs & 0.09044 \\
\hline Wtcol-Anpbs & $6.6 \mathrm{e}-05$ \\
\hline Wtpbs-Anpcol & $6.5 \mathrm{e}-05$ \\
\hline Wtcol-Anpcol & $\mathbf{0 . 0 0 9 6 0}$ \\
\hline Wtcol-Wtpbs & 0.00011 \\
\hline
\end{tabular}

Suppl-Fig-1D: Comparison of knee joints from WT and Anp32a ${ }^{/-}$mice injected with papain, by OARSI severity grade score

Research question: is the severity of articular cartilage damage (OARSI grade severity score) different between WT and ANPKO mice?

Null hypothesis: no difference in OARSI severity grade score between WT and ANPKO mice.

Assumptions: independent groups - normal distribution with unequal variances

1) model scores in genetically identical mice are commonly normally distributed

2) $\mathrm{QQ}$ (quantile-quantile) plots deviations from normality (controls with score 0 ).

3) Shapiro-Wilk test: WTPBS $W=0.70102$, $p$-value $=0.0002$

WTpap $\mathrm{W}=0.96016$, $\mathrm{p}$-value $=0.6646$

ANPPBS $W=0.70835, p$-value $=0.0003057$

ANPKOpap $\mathrm{W}=0.81436, \mathrm{p}$-value $=0.005654$ (low score outliers)

$\underline{\text { OARSI score (WTPBS-WTpap-ANPKOPBS-ANPKO pap) }}$
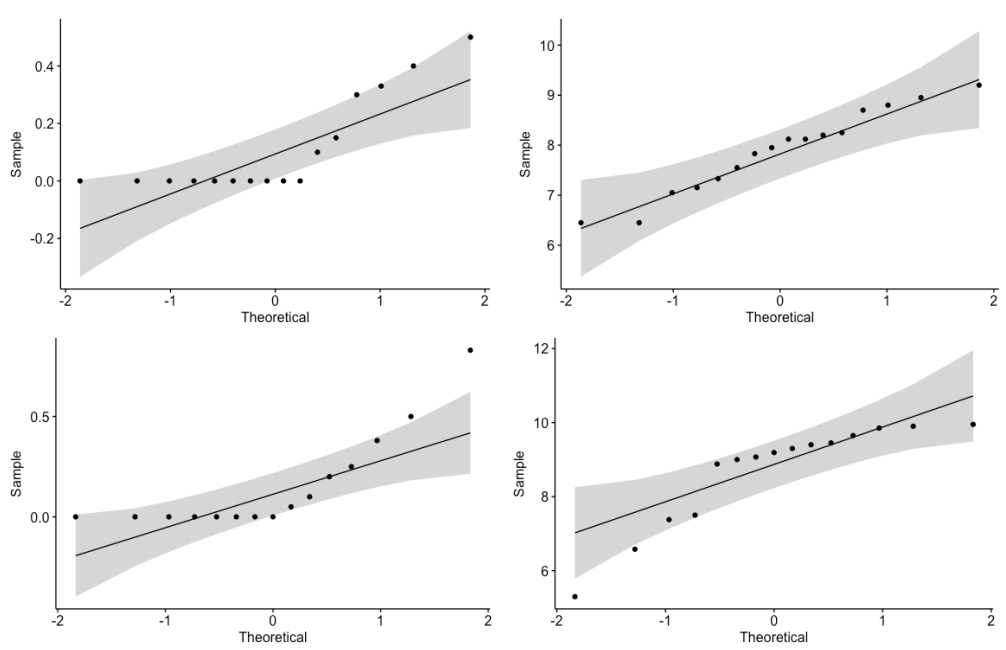

4) Unequal variances: Levene test F-value $=6.1396 p$-value $=0.001067$

Analysis selected: One-way Welch ANOVA (unequal variances) :

$F=601.13, p$-value $=2.2 e-16$

Post ANOVA pair-wise Welch t-tests with Bonferroni multi-comparison correction 


\begin{tabular}{|l|c|c|c|}
\hline Groups & p-value (corrected) & \multicolumn{2}{|c|}{ Estimates 95\% Cl of diff between means } \\
\hline Anppap-Anppbs & $2 \mathrm{e}-16$ & 7.73 & 9.348 \\
\hline Wtpbs-Anppap & $2 \mathrm{e}-16$ & -9.378 & -7.786 \\
\hline Wtpap-Anppbs & $2 \mathrm{e}-16$ & 6.931 & 8.524 \\
\hline Wtpbs-Anppbs & 1.000 & -0.839 & 0.7535 \\
\hline Wtpap-Anppap & 0.043 & -1.608 & -0.0158 \\
\hline Wtpbs-Wtpap & $2 \mathrm{e}-16$ & -8.553 & -6.987 \\
\hline
\end{tabular}

Sensitivity analysis of robustness:

IF normal distribution not assumed:

Kruskal-Wallis test: $\quad 48.567, \mathrm{df}=3, \mathrm{p}$-value $=1.613 \mathrm{e}-10$

Post KW pair-wise Mann-Whitney tests with Bonferroni multicomparison correction

\begin{tabular}{|l|c|}
\hline Groups & p-value (corrected) \\
\hline Anppap-Anppbs & $1.6 \mathrm{e}-05$ \\
\hline Wtpbs-Anppbs & 1.000 \\
\hline Wtpap-Anpbs & $1.1 \mathrm{e}-05$ \\
\hline Wtpbs-Anppap & $9.3 \mathrm{e}-06$ \\
\hline Wtpap-Anppap & $\mathbf{0 . 0 8 1}$ \\
\hline Wtpap-Wtpbs & $6.3 \mathrm{e}-06$ \\
\hline
\end{tabular}

\section{Supplementary Figure 2}

Suppl-Fig-2A. Absence of significant changes in chondrocyte marker expression in the articular cartilage of 8-week old Anp32a-deficient mice.

Research question: is the gene expression of Col2 and ACAN different between WT and ANPKO mice Null hypothesis: no difference in Col 2 and ACAN expression between WT and ANPKO mice.

Assumptions: independent groups - normal distribution with similar variances

1) Gene expression in tissue samples is commonly normally distributed

2) QQ (quantile-quantile) plots suggest normal distribution

Col2 - ACAN (WT - ANPKO)
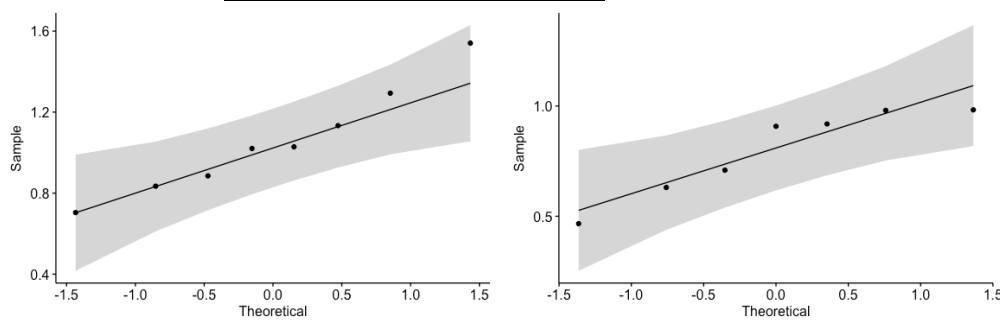

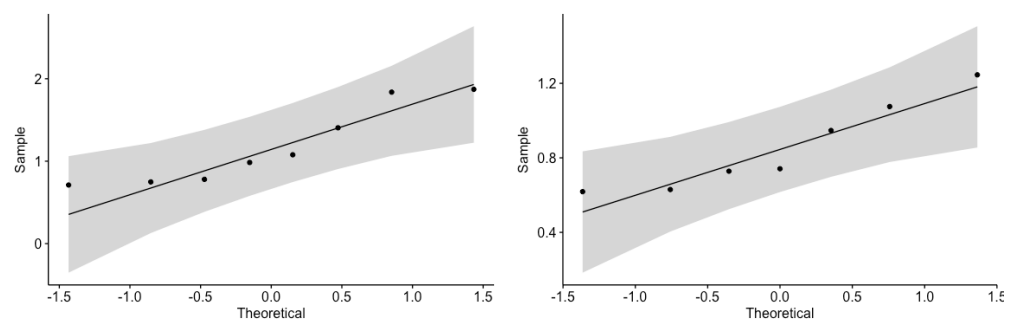

\begin{tabular}{|l|c|c|c|c|c|}
\hline \multicolumn{2}{|c|}{ Analysis selected: Two independent sample t-test (equal variance) } \\
\hline $\begin{array}{c}\text { ANPKO vs } \\
\text { WT }\end{array}$ & $\begin{array}{c}\text { Shapiro-Wilk test } \\
\text { WT }\end{array}$ & $\begin{array}{c}\text { Shapiro-Wilk test } \\
\text { ANPKO }\end{array}$ & Levene test & p-value & $\begin{array}{c}\mathbf{9 5 \% ~ C l} \text { of diff } \\
\text { of } \text { means }\end{array}$ \\
\hline Col2a1 & $\mathrm{W}=0.85584, \mathrm{p}-$ & $\mathrm{W}=0.89749, \mathrm{p}-$ & F-value: 2.0059 & $\mathbf{p}$-value $=$ & $\begin{array}{c}-\mathbf{0 . 7 5 2 0 4 7 0} \text { to } \\
\end{array}$ \\
& value $=0.1091$ & value $=0.316$ & $\mathrm{p}$-value: 0.1802 & $\mathbf{0 . 1 2 9 7}$ & $\mathbf{0 . 1 0 7 9 9 0 1}$ \\
\hline ACAN & $\mathrm{W}=0.96418, \mathrm{p}-$ & $\mathrm{W}=0.87142, \mathrm{p}-$ & F-value: 0.2147 & $\mathbf{p}$-value $=$ & $\mathbf{- 0 . 5 2 2 5 6 5 0 6}$ \\
& value $=0.8489$ & value $=0.191$ & $\mathrm{p}$-value: 0.6507 & $\mathbf{0 . 0 5 9 1 5}$ & $\mathbf{0 . 0 1 1 4 1 7 6 6}$ \\
\hline
\end{tabular}

Sensitivity analysis: Normal distribution not assumed:

Col2a1: Mann-Witney-test: $W=14, p$-value $=0.1206$

Acan: Mann-Witney-test: $W=13, p$-value $=0.09386$

Suppl-Fig-2B. human healthy articular chondrocytes with siRNA mediated silencing of $A N P 32 A$

No specific statistical analysis was used for these molecular biology analyses, but the evidence is based on the replication of the effect in an independent sample. Data shown are of three technical replicates with two data points obtained from two independent experiments.

\section{Supplementary Figure 4}

Research question: are compensatory mechanisms to contain increased oxidative stress active in ANPKO mice? Null hypothesis: no difference in the expression of different group between WT and ANPKO mice.

Assumptions: normal distribution

1) Gene expression in tissue samples is commonly normally distributed in genetically identical animals

2) QQ (quantile-quantile) plots suggest normal distribution

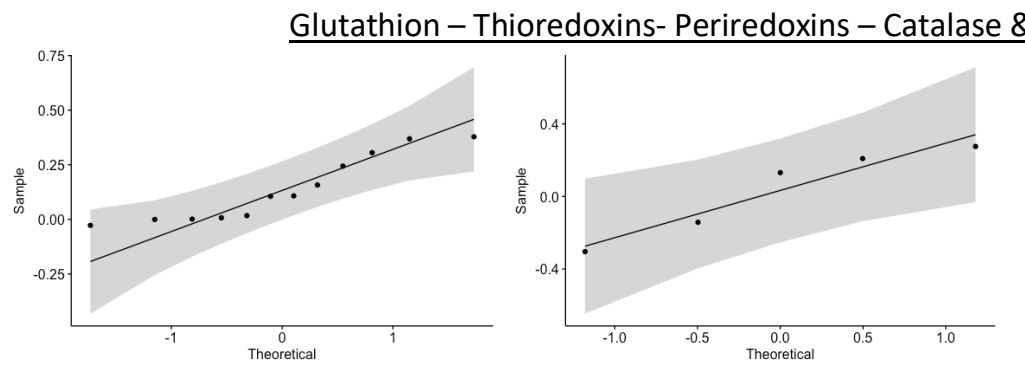



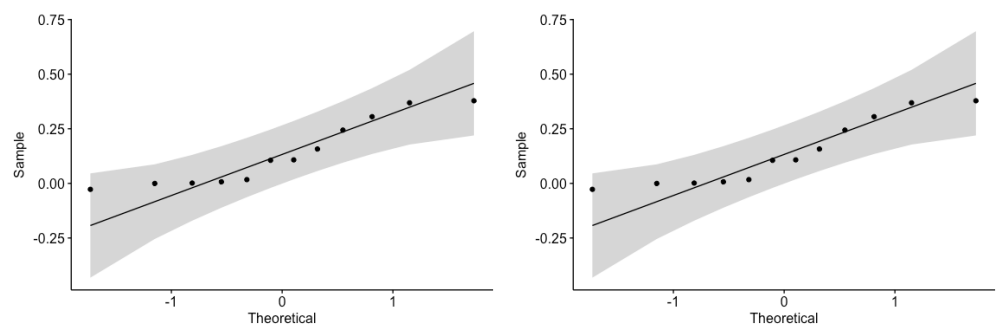

\begin{tabular}{|l|c|c|c|}
\hline \multicolumn{3}{|c|}{ Analysis selected: One-sample t-test with theoretical mean = 0 } \\
\hline \multicolumn{1}{|c|}{ Group } & $\begin{array}{c}\text { Shapiro-Wilk test } \\
\text { ANPKO }\end{array}$ & p-value & $\begin{array}{c}\text { Estimate: } \mathbf{9 5 \%} \\
\text { Cl of means }\end{array}$ \\
\hline glutathion & $\mathrm{W}=0.8733, \mathrm{p}-$ & $\mathbf{p}$-value $=$ & $\mathbf{0 . 0 4 3 2}$ to \\
& value $=0.0719$ & $\mathbf{0 . 0 0 8 5}$ & $\mathbf{0 . 2 3 4 6}$ \\
\hline thioredoxin & $\mathrm{W}=0.9062, \mathrm{p}-$ & $\mathrm{p}$-value & --0.2727 to \\
& value $=0.4453$ & 0.7739 & 0.3406 \\
\hline perioredoxin & $\mathrm{W}=0.97 \mathrm{è}, \mathrm{p}-$ & $\mathrm{p}$-value & -0.1297 to \\
& value $=0.9435$ & 0.4540 & 0.2559 \\
\hline catalase \& & $\mathrm{W}=0.9396, \mathrm{p}-$ & $\mathrm{p}$-value $=$ & -0.651 to \\
dismutases & value $=0.6518$ & 0.4409 & 0.3675 \\
\hline
\end{tabular}

Sensitivity analysis of robustness:

IF normal distribution not assumed:

\begin{tabular}{|l|c|}
\hline \multicolumn{2}{|l|}{ Analysis selected: One-sample Wilcoxon signed rank test with } \\
\hline \multicolumn{1}{|l|}{ theoretical mean $\mathbf{~} \mathbf{0}$} \\
\hline glutathion & p-value \\
\hline thioredoxin & p-value $=\mathbf{0 . 0 0 6 8}$ \\
& p-value $=1$ \\
\hline perioredoxin & p-value $=0.4688$ \\
\hline catalase \& dismutases & p-value $=0.6250$ \\
\hline
\end{tabular}

\section{Supplementary Figure 5}

Suppl-Fig-5B: Quantification of type X collagen staining in the growth plate from Anp32a $a^{-/}$mice compared to WT mice, with or without NAC treatment, by digital image analysis $(\mathrm{n}=3$ per group)

Research question: Are type X collagen levels increased in the growth plate of ANPKO mice compared to wildtype mice and what is the effect of NAC treatment hereon?

Null hypothesis: 1 . no difference in type $X$ collagen staining between ANPKO and WT mice

2. no effect of NAC on type $X$ collagen staining in ANPKO mice

Assumptions: independent groups - normal distribution with unequal variances

1) Protein levels in genetically identical mice are commonly normally distributed

2) QQ (quantile-quantile) plots suggest normality).

3) Shapiro-Wilk test: WT: reference values

ANPKO: $\mathrm{W}=0.90147, \mathrm{p}$-value $=0.3902$

WTNAC: $\mathrm{W}=0.88534, \mathrm{p}$-value $=0.3402$

ANPKONAC: $\mathrm{W}=0.92229, \mathrm{p}$-value $=0.4604$

ColX levels (WT (ref 100\%) -ANPKO -WTNAC - ANPKO NAC) 

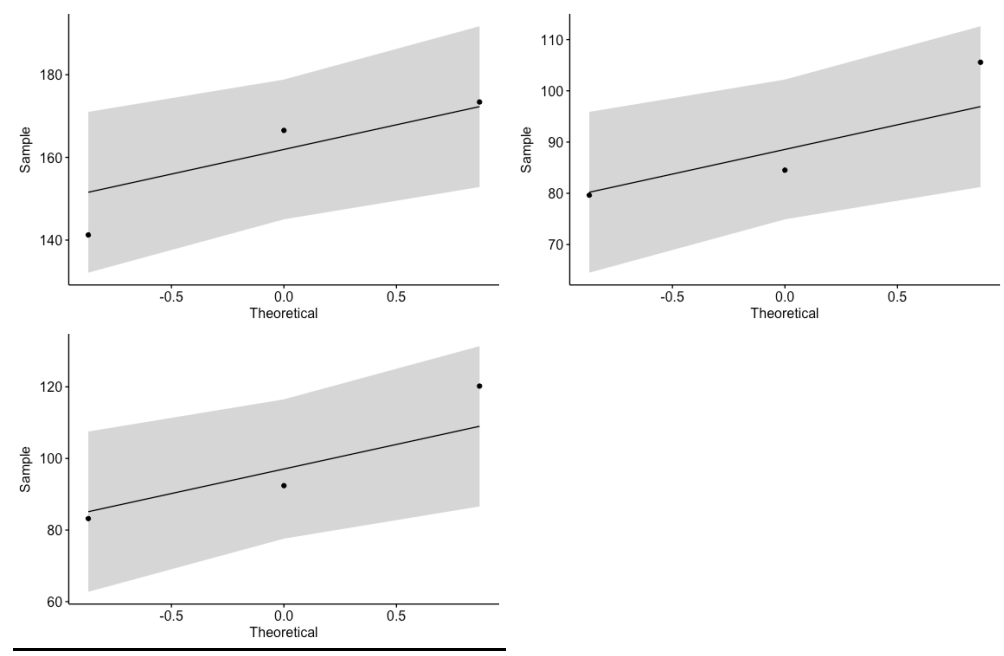

4) Equal variances: Levene's test F-value $=0.7349-\mathrm{P}$-value $=0.56$

\section{Analysis selected: One-way ANOVA:}

$F=14.87$, $p$-value $=0.00123$

\begin{tabular}{|l|c|c|c|}
\hline \multicolumn{5}{|l|}{ Post ANOVA pair-wise t-tests with Bonferroni multi-comparison correction } \\
\hline Groups & $\mathbf{p}$-value (corrected) & Estimates $\mathbf{9 5 \%}$ Cl of diff between means \\
\hline ANPKO-ANPKONAC & $\mathbf{0 , 0 0 5 0}$ & $\mathbf{2 0 . 4 3}$ & $\mathbf{1 0 3 . 1}$ \\
\hline WT-ANPKO & $\mathbf{0 , 0 0 5 7}$ & $\mathbf{- 1 0 1 . 7}$ & $\mathbf{- 1 9 . 0 3}$ \\
\hline WTNAC-ANPKO & 0.0021 & -111.8 & -29.12 \\
\hline WT-ANPKONAC & 0.999 & -31.96 & 42.76 \\
\hline WTNAC-ANPKONAC & 1 & -50.05 & 32.67 \\
\hline WT-WTNAC & 1 & -31.27 & 51.45 \\
\hline
\end{tabular}

Sensitivity analysis of robustness: analysis not confirmed

Kruskal-Wallis test: $\quad$ chi-squared $=6.7872, \mathrm{df}=3, \mathrm{p}$-value $=\mathbf{0 . 0 5 4}$

\section{Supplementary Figure 7}

Supp-Fig-7B-C: The average stride length and regularity were different for Anp32a-deficient mice than for WT mice in both front-paw (FP) and hind-paw (HP), a defect that was rescued by NAC treatment.

Research question: Can gait parameters (stride length), different between WT and ANPKO mice (Fig 5) be rescued by NAC treatment

Null hypothesis: no effect of late onset NAC treatment on stride length in ANPKO mice

Assumptions: independent groups - normal distribution with equal variances (see fig 5)

Missing data: one observation in WTNONAC group carried forward from month 10 to 12 .

$\underline{\text { Frontlimbs }}$

Analysis selected: Two-way ANOVA with repeated measurements (different timepoints): 
$\underline{\text { Hindlimbs }}$

\begin{tabular}{|l|c|c|}
\hline \multicolumn{1}{|c|}{ Post ANOVA pair-wise t-tests with Bonferroni multi-comparison correction } \\
\hline \multicolumn{1}{|c|}{ Groups } & p-value & $\begin{array}{c}\text { Estimates } \mathbf{9 5 \%} \mathrm{Cl} \text { of diff } \\
\text { between means }\end{array}$ \\
\hline WT NAC vs. WT no NAC & 0,0974 & $-328,7$ to 22,52 \\
\hline WT NAC vs. KO NAC & 0,1111 & $-22,48$ to 271,4 \\
\hline WT NAC vs. KO no NAC & 0,0003 & 197,1 to 511,3 \\
\hline WT no NAC vs. KO NAC & 0,0024 & 110,9 to 444,1 \\
\hline WT no NAC vs. KO no NAC & $<\mathbf{0 , 0 0 0 1}$ & $\mathbf{3 3 1 , 7}$ to $\mathbf{6 8 2 , 9}$ \\
\hline KO NAC vs. KO no NAC & $\mathbf{0 , 0 0 3 7}$ & $\mathbf{8 2 , 8 3}$ to $\mathbf{3 7 6 , 7}$ \\
\hline
\end{tabular}

Analysis selected: Two-way ANOVA with repeated measurements (different timepoints):

Interaction between treatment and genotype $p$-value $=0,0003$

Effect of time

$p$-value $=0,0016$

Effect of genotype

$p$-value $=<0,0001$

\begin{tabular}{|l|c|c|}
\hline \multicolumn{1}{|c|}{ Post ANOVA pair-wise t-tests with Bonferroni multi-comparison correction } \\
\hline Groups & p-value & $\begin{array}{c}\text { Estimates } \mathbf{9 5 \%} \mathrm{Cl} \text { of diff } \\
\text { between means }\end{array}$ \\
\hline WT NAC vs. WT no NAC & 1 & $-224,2$ to 96,84 \\
\hline WT NAC vs. KO NAC & 0,0079 & 51,84 to 320,5 \\
\hline WT NAC vs. KO no NAC & $<0,0001$ & 342,8 to 629,9 \\
\hline WT no NAC vs. KO NAC & 0,0027 & 97,55 to 402,2 \\
\hline WT no NAC vs. KO no NAC & $<\mathbf{0 , 0 0 0 1}$ & $\mathbf{3 8 9 , 5}$ to $\mathbf{7 1 0 , 6}$ \\
\hline KO NAC vs. KO no NAC & $\mathbf{0 , 0 0 0 3}$ & $\mathbf{1 6 5 , 9}$ to $\mathbf{4 3 4 , 5}$ \\
\hline
\end{tabular}

Regularity index

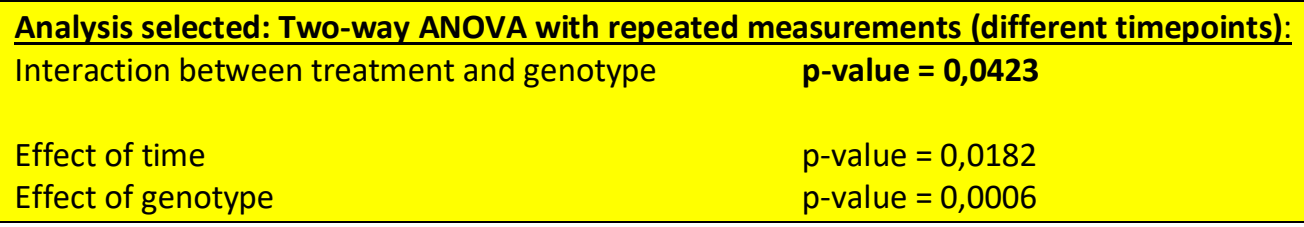

\begin{tabular}{|l|c|c|}
\hline \multicolumn{1}{|c|}{ Post ANOVA pair-wise t-tests with Bonferroni multi-comparison correction } \\
\hline Groups & p-value & $\begin{array}{c}\text { Estimates } \mathbf{9 5 \%} \text { Cl of diff } \\
\text { between means }\end{array}$ \\
\hline WT NAC vs. WT no NAC & 1 & $-6,637$ to 7,905 \\
\hline WT NAC vs. KO NAC & 0,4587 & $-2,528$ to 9,639 \\
\hline WT NAC vs. KO no NAC & 0,0009 & 5,991 to 19 \\
\hline WT no NAC vs. KO NAC & 1 & $-3,976$ to 9,82 \\
\hline WT no NAC vs. KO no NAC & $\mathbf{0 , 0 0 2 8}$ & $\mathbf{4 , 5 8 9}$ to $\mathbf{1 9 , 1 3}$ \\
\hline KO NAC vs. KO no NAC & $\mathbf{0 , 0 0 5 5}$ & $\mathbf{2 , 8 5 5}$ to $\mathbf{1 5 , 0 2}$ \\
\hline
\end{tabular}

Sensitivity analysis 
One-way ANOVA tests at time-point 10 months using 4 groups

Frontlimbs: $F=10,31$ p-value $=\mathbf{0 , 0 0 4 0}$

\begin{tabular}{|l|c|c|}
\hline \multicolumn{1}{|c|}{ Post ANOVA pair-wise t-tests with Bonferroni multi-comparison correction } \\
\hline Groups & $\mathbf{p}$-value & $\begin{array}{c}\text { Estimates } \mathbf{9 5 \%} \text { Cl of diff } \\
\text { between means }\end{array}$ \\
\hline WT NAC vs. WT no NAC & 0,6821 & $-540,9$ to 175,3 \\
\hline WT NAC vs. KO NAC & 0,4905 & -471 to 128,2 \\
\hline WT NAC vs. KO no NAC & 0,1192 & $-53,24$ to 587,4 \\
\hline WT no NAC vs. KO NAC & 1 & $-328,4$ to 351,1 \\
\hline WT no NAC vs. KO no NAC & $\mathbf{0 , 0 1 4 3}$ & $\mathbf{9 1 , 7 5}$ to $\mathbf{8 0 8}$ \\
\hline KO NAC vs. KO no NAC & $\mathbf{0 , 0 0 5 6}$ & $\mathbf{1 3 8 , 9}$ to $\mathbf{7 3 8 , 1}$ \\
\hline
\end{tabular}

Normal distribution not assumed:

Kruskal Wallis-test: $\mathrm{W}=\mathbf{8 , 7}$ - $\mathbf{p}$-value $\mathbf{=} \mathbf{0 . 0 0 5 5}$

Hindlimbs: $F=15,34-\mathbf{p}$-value $=\mathbf{0 , 0 0 1 1}$

\begin{tabular}{|l|c|c|}
\hline \multicolumn{1}{|c|}{ Post ANOVA pair-wise t-tests with Bonferroni multi-comparison correction } \\
\hline WT NAC vs. WT no NAC & p-value & $\begin{array}{c}\text { Estimates } 95 \% \text { Cl of diff } \\
\text { between means }\end{array}$ \\
\hline WT NAC vs. KO NAC & $>0,9999$ & $-565,3$ to 328,1 \\
\hline WT NAC vs. KO no NAC & $>0,9999$ & $-474,2$ to 273,2 \\
\hline WT no NAC vs. KO NAC & 0,0076 & 157,5 to 956,6 \\
\hline WT no NAC vs. KO no NAC & $>0,9999$ & $-405,6$ to 441,9 \\
\hline KO NAC vs. KO no NAC & 0,0046 & 229 to 1122 \\
\hline
\end{tabular}

Normal distribution not assumed:

Kruskal Wallis-test: $W=7,67, \mathbf{p}$-value $=\mathbf{0 . 0 2 1 7}$

Regularity index: $F=0,9-p$-value $=\mathbf{0 , 0 0 3 4}$

\begin{tabular}{|l|c|c|}
\hline \multicolumn{1}{|c|}{ Post ANOVA pair-wise t-tests with Bonferroni multi-comparison correction } \\
\hline Groups & p-value & $\begin{array}{c}\text { Estimates } \mathbf{9 5 \%} \text { Cl of diff } \\
\text { between means }\end{array}$ \\
\hline WT NAC vs. WT no NAC & 1 & $-14,79$ to 14,64 \\
\hline WT NAC vs. KO NAC & 1 & $-12,13$ to 12,49 \\
\hline WT NAC vs. KO no NAC & 0,0094 & 4,561 to 30,88 \\
\hline WT no NAC vs. KO NAC & 1 & $-13,7$ to 14,22 \\
\hline WT no NAC vs. KO no NAC & $\mathbf{0 , 0 1 7 8}$ & $\mathbf{3 , 0 8 4}$ to $\mathbf{3 2 , 5 1}$ \\
\hline KO NAC vs. KO no NAC & $\mathbf{0 , 0 0 6 7}$ & $\mathbf{5 , 2 2 7}$ to $\mathbf{2 9 , 8 5}$ \\
\hline
\end{tabular}

Normal distribution not assumed:

Kruskal Wallis-test: $W=6,52, p$-value $=0.0619$ 


\section{Supplementary Table 3}

Supp-Table-3: Gait paramters in Anp32a-deficient mice compared to WT mice

Research question: Are gait parameters different between WT and ANPKO mice and what is the effect of NAC on such differences?

Null hypothesis: 1 no difference in stride length between ANPKO and WT mice

2. no effect of NAC on stride length in ANPKO mice

Assumptions: independent groups - normal distribution with equal variances (cfr. Figure 5 - normality and variance test performed as above)

$\underline{\text { Front limbs }}$

\begin{tabular}{|c|c|c|}
\hline \multicolumn{3}{|l|}{ One-way Anova tests } \\
\hline Parameter & 8 weeks & 16 weeks \\
\hline Intensity & $W=13.45 p$-value $=0.0001$ & $W=7.034 p$-value $=0.0027$ \\
\hline Stand & $W=10,24 p$-value $=0.0004$ & $W=14,64 p$-value $=0.0001$ \\
\hline Paw angle & $W=3.121 p$-value $=0.0518$ & $W=3.814 p$-value $=0.0281$ \\
\hline Duty cycle (\% stance) & $W=11.11 p$-value $=0.002$ & $W=4.532 p$-value $=0.0155$ \\
\hline Swing speed & $W=12.58 p$-value $=0.0001$ & $W=4.098 p$-value $=0.0221$ \\
\hline Base of contact & $\mathrm{W}=2,116 \mathrm{p}$-value $=0.1339$ & $W=3.6 p$-value $=0.0339$ \\
\hline
\end{tabular}

\begin{tabular}{|c|c|c|}
\hline \multicolumn{3}{|c|}{ Post ANOVA pair-wise t-tests with Bonferroni multi-comparison correction } \\
\hline Groups & p-value & $\begin{array}{c}\text { Estimates } 95 \% \mathrm{Cl} \text { of diff } \\
\text { between means }\end{array}$ \\
\hline \multicolumn{3}{|l|}{ Intensity 8 weeks } \\
\hline WT vs. ANPKO & 0,0266 & 0,353 to 7,591 \\
\hline ANPKO vs. ANPKONAC & 0,0171 & 0,5979 to 7,836 \\
\hline \multicolumn{3}{|l|}{ Intensity 16 weeks } \\
\hline WT vs. ANPKO & 0,3295 & $-1,644$ to 9,061 \\
\hline ANPKO vs. ANPKONAC & 1 & $-6,46$ to 4,245 \\
\hline \multicolumn{3}{|l|}{ Stand 8 weeks } \\
\hline WT vs. ANPKO & 0,0345 & $-0,121$ to $-0,003366$ \\
\hline ANPKO vs. ANPKONAC & 0,0003 & 0,04737 to 0,165 \\
\hline \multicolumn{3}{|l|}{ Stand 16 weeks } \\
\hline WT vs. ANPKO & 0,0006 & $-0,1552$ to $-0,03928$ \\
\hline ANPKO vs. ANPKONAC & $<0,0001$ & 0,05928 to 0,1752 \\
\hline \multicolumn{3}{|l|}{ Duty cycle 8 weeks } \\
\hline WT vs. ANPKO & 0,5307 & $-11,76$ to 2,868 \\
\hline ANPKO vs. ANPKONAC & 0,0002 & 6,434 to 21,06 \\
\hline \multicolumn{3}{|l|}{ Duty cycle 16 weeks } \\
\hline WT vs. ANPKO & 0,4164 & $-12,69$ to 2,675 \\
\hline ANPKO vs. ANPKONAC & 0,0229 & 0,9265 to 16,29 \\
\hline \multicolumn{3}{|l|}{ Base of contact 16 weeks } \\
\hline WT vs. ANPKO & 0,0001 & 2,556 to 7,706 \\
\hline ANPKO vs. ANPKONAC & 0,0096 & $-5,798$ to $-0,6484$ \\
\hline \multicolumn{3}{|l|}{ Swing speed 8 weeks } \\
\hline WT vs. ANPKO & 0.0001 & 2,556 to 7,706 \\
\hline
\end{tabular}




\begin{tabular}{|l|c|c|}
\hline ANPKO vs. ANPKONAC & $\mathbf{0 . 0 0 9 6}$ & $-5,798$ to $-0,6484$ \\
\hline Swing speed 16 weeks & & \\
\hline WT vs. ANPKO & 0.2760 & $-1,018$ to 6,342 \\
\hline ANPKO vs. ANPKONAC & 0.0613 & $-7,243$ to 0,1172 \\
\hline
\end{tabular}

$\underline{\text { Hind limbs }}$

\begin{tabular}{|c|c|c|}
\hline \multicolumn{3}{|l|}{ One-way Anova tests } \\
\hline Parameter & 8 weeks & 16 weeks \\
\hline Intensity & $\mathrm{W}=21.02 \mathrm{p}$-value $=0.0001$ & $\mathrm{~W}=6.008 \mathrm{p}$-value $=0.0051$ \\
\hline Stand & $W=11.31 p$-value $=0.0002$ & $\mathrm{~W}=13.77 \mathrm{p}$-value $=0.0001$ \\
\hline Paw angle & $W=5.449 p$-value $=0.0074$ & $W=2.635 p$-value $=0.0798$ \\
\hline Duty cycle (\% stance) & $W=11.17 p$-value $=0.0002$ & $W=4.175 p$-value $=0.0208$ \\
\hline Swing speed & $\mathrm{W}=2.522 \mathrm{p}$-value $=0.0904$ & $W=4.116 p-$ value $=0.0218$ \\
\hline Base of contact & $W=7.762 p$-value $=0.0016$ & $W=1.992 p$-value $=0.1513$ \\
\hline
\end{tabular}

\begin{tabular}{|c|c|c|}
\hline \multicolumn{3}{|c|}{ Post ANOVA pair-wise t-tests with Bonferroni multi-comparison correction } \\
\hline Groups & p-value & $\begin{array}{c}\text { Estimates } 95 \% \mathrm{Cl} \text { of diff } \\
\text { between means }\end{array}$ \\
\hline \multicolumn{3}{|l|}{ Intensity 8 weeks } \\
\hline WT vs. ANPKO & 0,1778 & $-1,157$ to 10,26 \\
\hline ANPKO vs. ANPKONAC & 0.0001 & 5,312 to 16,73 \\
\hline \multicolumn{3}{|l|}{ Intensity 16 weeks } \\
\hline WT vs. ANPKO & 0,6833 & $-4,484$ to 15,94 \\
\hline ANPKO vs. ANPKONAC & 1 & $-10,36$ to 10,06 \\
\hline \multicolumn{3}{|l|}{ Stand 8 weeks } \\
\hline WT vs. ANPKO & 0,0055 & $-0,166$ to $-0,02394$ \\
\hline ANPKO vS. ANPKONAC & 0,0002 & 0,05861 to 0,2006 \\
\hline \multicolumn{3}{|l|}{ Stand 16 weeks } \\
\hline WT vs. ANPKO & 0,0013 & $-0,1705$ to $-0,03709$ \\
\hline ANPKO vs. ANPKONAC & 0,0001 & 0,06376 to 0,1972 \\
\hline \multicolumn{3}{|l|}{ Paw angle 8 weeks } \\
\hline WT vs. ANPKO & 0,193 & $-28,72$ to 3,485 \\
\hline ANPKO vs. ANPKONAC & 1 & $-9,096$ to 23,11 \\
\hline \multicolumn{3}{|l|}{ Duty cycle 8 weeks } \\
\hline WT vs. ANPKO & 0,1094 & $-12,95$ to 0,8528 \\
\hline ANPKO vs. ANPKONAC & 0,0001 & 6,389 to 20,19 \\
\hline \multicolumn{3}{|l|}{ Duty cycle 16 weeks } \\
\hline WT vs. ANPKO & 0,2787 & $-14,51$ to 2,344 \\
\hline ANPKO vs. ANPKONAC & 0,0181 & 1,314 to 18,16 \\
\hline \multicolumn{3}{|l|}{ Swing speed 16 weeks } \\
\hline WT vs. ANPKO & 0.3970 & $-1,414$ to 6,907 \\
\hline ANPKO vs. ANPKONAC & 0,0257 & $-8,748$ to $-0,4276$ \\
\hline \multicolumn{3}{|l|}{ Base of contact 8 weeks } \\
\hline WT vs. ANPKO & 0.0023 & -767 to $-145,4$ \\
\hline ANPKO vs. ANPKONAC & 0,0365 & 15,16 to 636,8 \\
\hline \multicolumn{3}{|c|}{ Base of contact 16 weeks } \\
\hline WT vs. ANPKO & 0.1756 & -1372 to 153 \\
\hline
\end{tabular}




\begin{tabular}{|l|c|c|}
\hline ANPKO vs. ANPKONAC & 1 & -421 to 1104 \\
\hline Swing speed 8 weeks & & $-0,6511$ to 6,351 \\
\hline WT vs. ANPKO & 0.1606 & $-5,182$ to 1,82 \\
\hline ANPKO vs. ANPKONAC & 1 & $-1,414$ to 6,907 \\
\hline Swing speed 16 weeks & & $-8,748$ to $-0,4276$ \\
\hline WT vs. ANPKO & 0.3970 & $\mathbf{0 . 0 2 5 7}$ \\
\hline ANPKO vs. ANPKONAC &
\end{tabular}

\section{References}

1. Ramos YF, den Hollander W, Bovee JV, Bomer N, van der Breggen R, Lakenberg N, et al. Genes involved in the osteoarthritis process identified through genome wide expression analysis in articular cartilage; the RAAK study. PLoS One. 2014;9(7):e103056.

2. Lodewyckx L, Cailotto F, Thysen S, Luyten FP, Lories RJ. Tight regulation of wingless-type signaling in the articular cartilage - subchondral bone biomechanical unit: transcriptomics in Frzb-knockout mice. Arthritis Res Ther. 2012;14(1):R16.

3. Monteagudo S, Cornelis FMF, Aznar-Lopez C, Yibmantasiri P, Guns LA, Carmeliet P, et al. DOT1L safeguards cartilage homeostasis and protects against osteoarthritis. Nat Commun. 2017;8. 